\title{
TOPOLOGY OF ANGLE VALUED MAPS, BAR CODES AND JORDAN BLOCKS.
}

\author{
DAN BURGHELEA AND STEFAN HALLER
}

\begin{abstract}
.
In this paper one presents a collection of results about the "bar codes" and "Jordan blocks" introduced in [3] as computer friendly invariants of a tame angle-valued map and one relates these invariants to the Betti numbers, Novikov Betti numbers and the monodromy of the underlying space and map. Among others, one organizes the bar codes as two configurations of points in $\mathbb{C} \backslash 0$ and one establishes their main properties: stability property and when the underlying space is a closed topological manifold, Poincaré duality property. One also provides an alternative computer friendly definition of the monodromy of an angle valued map based on the algebra of linear relations as well as a refinement of Morse and Morse-Novikov inequalities.
\end{abstract}

\section{Contents}

1. The results

2. Graph representations

3. Bar codes and Jordan blocks via graph representations 21

4. Proof of Theorem 1.1 and some refinements. 25

5. Stability for configurations $C_{r}(f)$. Proof of Theorem 1.2

6. Poincaré duality for configurations $C_{r}(f)$. Proof of Theorem 1.3

7. The mixed bar codes. Proof of Theorem 1.5 44

8. Linear relations and monodromy. Proof of Theorem 1.4

9. Proof of Theorem 1.7

Appendices

Appendix A. An example 59

Appendix B. More examples 60

Appendix C. Structure of finitely generated modules over principal ideal domains 63

References 64

Date: September 17, 2018.

Part of this work was done while the second author enjoyed the hospitality of the Ohio State University. The present version of this paper was finalized while first author was visiting the Bernoulli Center at EPFL Lausanne and MPIM Bonn. The first author acknowledges partial support from NSF grant MCS 0915996. The second author acknowledges the support of the Austrian Science Fund, grant P19392-N13. 


\section{The RESUlts}

In this paper a nice space is a friendlier name for a locally compact ANR (Absolute Neighborhood Retract) ${ }^{1}$ Finite dimensional simplicial complexes and finite dimensional topological manifolds are nice spaces but the class is considerably larger. A tame map is a proper continuous map $f: X \rightarrow \mathbb{R}$ or $f: X \rightarrow \mathbb{S}^{1}$, defined on a nice space $X$, which satisfies:

(i) each fiber of $f$ is a neighborhood deformation retract, and

(ii) away from a discrete set $\Sigma \subset \mathbb{R}$ or $\Sigma \subset \mathbb{S}^{1}$ the restriction of $f$ to $X \backslash f^{-1}(\Sigma)$ is a fibration, cf. [3]. In particular for $t \notin \Sigma(f)$ there exists a neighborhood $U \ni t$ such that for any $t^{\prime} \in U$, the inclusion $f^{-1}\left(t^{\prime}\right) \subset f^{-1}(U)$ is a homotopy equivalence.

All proper simplicial maps and proper smooth generic maps defined on a smooth manifold $]^{2}$ in particular proper real or angle valued Morse maps, are tame. At least for spaces homeomorphic to simplicial complexes the set of tame maps is residual in the space of all continuous maps and weakly homotopy equivalent to the space of all continuous maps (equipped with compact open topology) $?^{3}$

Most of the time we will have an a priory fixed field $\kappa$ and homology, Novikov homology, Betti numbers, etc. will be considered with respect to this field. For simplicity in writing, the field $\kappa$ will be omitted from the notations.

In this paper we consider a tame map, $f: X \rightarrow \mathbb{S}^{1}$, and as in [3], one associates to the map $f$ :

(i) the set of critical angles $0<\theta_{1}<\theta_{2}<\cdots<\theta_{m} \leq 2 \pi$,

(ii) for any $r=0,1, \ldots, \operatorname{dim} X$, four types of intervals of real numbers,

(1) closed $([a, b])$,

(2) open $((a, b))$,

(3) closed-open $([a, b))$,

(4) open-closed $((a, b])$,

subsequently called $r$-bar codes, whose ends $\bmod 2 \pi$ are critical angles, with $0<a \leq 2 \pi$

(iii) for any $r=0,1, \ldots, \operatorname{dim} X$, a collection of isomorphism classes of indecomposable pairs $J=\left(V_{J}, T_{J}\right)$, where $T_{J}$ is a linear automorphism of a finite dimensional $\kappa$-vector space $V_{J}$, subsequently called Jordan blocks.

The bar codes can be also regarded as equivalence classes of intervals as above modulo translation by an integer multiple of $2 \pi$, with ends $\bmod 2 \pi$ critical angles.

Recall that a pair $(V, T)$ is indecomposable if not isomorphic to the sum of two nontrivial pairs. In this case if $T$ has $\lambda \in \kappa$ as an eigenvalue all other eigenvalues are equal to $\lambda$, and $(V, T)$ is isomorphic to $\left(\kappa^{k}, T(\lambda, k)\right)$ where $T(\lambda, k)$ is the $k \times k$

\footnotetext{
${ }^{1} \mathrm{~A}$ metrizable, locally compact, finite dimensional locally contractible space is nice, see [23].

${ }^{2}$ Here "generic" means that for any $x \in M$ the quotient algebra of germs of smooth functions at $x$ by the ideal of partial derivatives is a finite dimensional vector space.

${ }^{3}$ In case that the space $X$ is homeomorphic to a finite dimensional simplicial complex, this is consequence of the approximability of continuous maps by pl-maps.
} 
matrix

$$
T(\lambda, k)=\left(\begin{array}{ccccc}
\lambda & 1 & 0 & \cdots & 0 \\
0 & \lambda & 1 & \ddots & \vdots \\
0 & 0 & \ddots & \ddots & 0 \\
\vdots & \ddots & \ddots & \lambda & 1 \\
0 & \cdots & 0 & 0 & \lambda
\end{array}\right)
$$

if $k \geq 2$, and $T(\lambda, 1)=(\lambda)$ if $k=1$. In [3] the indecomposable pairs $\left(\kappa^{k}, T(\lambda, k)\right)$ were called Jordan cells. When $\kappa$ is algebraically closed all Jordan blocks are Jordan cells.

We denote by $\mathcal{B}_{r}^{c}(f), \mathcal{B}_{r}^{o}(f), \mathcal{B}_{r}^{c o}(f), \mathcal{B}_{r}^{o c}(f)$ the collections of closed, open, closedopen and open-closed $r$-bar codes and by $\mathcal{J}_{r}(f)$ the collection of $r$-Jordan blocks. For brevity we also write $\mathcal{B}_{r}(f):=\mathcal{B}_{r}^{c}(f) \sqcup \mathcal{B}_{r}(f) \sqcup \mathcal{B}_{r}(f) \sqcup \mathcal{B}_{r}(f)$. Each bar code or Jordan block appears with a multiplicity possibly larger than one. All these collections are multisets which means each element appears with multiplicity. For $u \in \kappa \backslash 0$ we denote by $\mathcal{J}_{r, u}(f)$ the sub-collection of $r$-Jordan blocks with eigenvalue $u$. In view of the definitions in Section 3, cf. also [3], each tame map has finitely many bar codes and Jordan blocks.

It was shown in [3] that for simplicial maps these invariants are effectively computable and an algorithm for their calculation was proposed. Existence of such algorithms is what we mean by computer friendly invariants. All these invariants are described in Section 3 ,

In order to formulate the results, we recall that any continuous map $f: X \rightarrow \mathbb{S}^{1}$ determines an integral cohomology class $\xi_{f} \in H^{1}(X ; \mathbb{Z})$ via pull back of a fixed generator in $H^{1}\left(\mathbb{S}^{1} ; \mathbb{Z}\right) \cong \mathbb{Z}$. By homotopy invariance, homotopic maps $f_{1}, f_{2}: X \rightarrow$ $\mathbb{S}^{1}$ determine the same class, $\xi_{f_{1}}=\xi_{f_{2}}$. If $X$ an ANR, then this assignment induces a bijection between the set of homotopy classes of maps $X \rightarrow \mathbb{S}^{1}$ and $H^{1}(X ; \mathbb{Z})$. In other words, any class $\xi \in H^{1}(X ; \mathbb{Z})$ is of the form $\xi=\xi_{f}$ for some continuous angle-valued map $f$ which is unique up to homotopy. This follows from the fact that the circle $\mathbb{S}^{1}$ is an Eilenberg-MacLane space $K(\mathbb{Z}, 1)$, see [21, Section 4.3].

We say that two pairs $\left(X_{1}, \xi_{1}\right)$ and $\left(X_{2}, \xi_{2}\right), \xi_{1}, \xi_{2} \in H^{1}(X ; \mathbb{Z})$, are homotopy equivalent if there exists a homotopy equivalence $\omega: X_{1} \rightarrow X_{2}$ s.t. $\omega^{*}\left(\xi_{2}\right)=\xi_{1}$.

The basic algebraic topology invariants associated with a pair $(X, \xi), \xi \in H^{1}(X ; \mathbb{Z})$, a field $\kappa$, and a positive integer $r \in \mathbb{N}_{0}$ we consider in this paper are:

(1) the singular homology $H_{r}(X)$, a $\kappa$-vector space whose dimension, when finite, is called the Betti number $\beta_{r}(X)$;

(2) the Novikov homology $H_{r}^{N}(X ; \xi)$, a vector space over the field of Laurant power series $\left.\kappa\left[t^{-1}, t\right]\right]$ with coefficients in $\kappa$, whose dimension, when finite, is called the Novikov-Betti number $\beta_{r}^{N}(X ; \xi)$; and

(3) the $r$-monodromy, an isomorphism class of pairs $\left(V_{r}, T_{r}\right)$ where $V_{r}$ is a $\kappa$-vector space and $T_{r}: V_{r} \rightarrow V_{r}$ is a linear isomorphism.

If $X$ is a compact ANR then $\beta_{r}(X), \beta_{r}^{N}(X ; \xi)$, and $\operatorname{dim}\left(V_{r}\right)$ are finite.

The first result we prove in this paper is Theorem 1.1 below.

Theorem 1.1 (Homotopy invariants). If $f: X \rightarrow \mathbb{S}^{1}$ is a tame map and $\xi_{f} \in$ $H^{1}(X ; \mathbb{Z})$ is the integral cohomology class represented by $f$ then: 
(a) $\sharp \mathcal{B}_{r}^{c}(f)+\sharp \mathcal{B}_{r-1}^{o}(f)$ is a homotopy invariant of the pair $\left(X, \xi_{f}\right)$, more precisely is equal to the Novikov-Betti number $\beta_{r}^{N}\left(X ; \xi_{f}\right)$.

(b) The collection $\mathcal{J}_{r}(f)$ is a homotopy invariant of the pair $\left(X, \xi_{f}\right)$. More precisely, $\bigoplus_{J \in \mathcal{J}_{r}(f)} J:=\bigoplus_{J \in \mathcal{J}_{r}(f)}\left(V_{J}, T_{J}\right)$ is the r-monodromy $\left(V_{r}, T_{r}\right)$ of $\left(X ; \xi_{f}\right)$. (c) $\sharp \mathcal{B}_{r}^{c}(f)+\sharp \mathcal{B}_{r-1}^{o}(f)+\sharp \mathcal{J}_{r, 1}(f)+\sharp \mathcal{J}_{r-1,1}(f)$ is equal to the Betti number $\beta_{r}(X)$.

The definition of Novikov-Betti numbers and of the monodromy are given in Section 4 and "H" denotes the cardinality of a multiset.

Item (c) has been already established in [3, Theorem 3.2] and is included in Theorem 1.1 only for the completeness of the topological information derived from bar codes and Jordan blocks.

In view of Theorem 1.1 it is natural to put together $\mathcal{B}_{r}^{c}(f)$ and $\mathcal{B}_{r-1}^{o}(f)$. For this purpose consider $\mathbb{T}=\mathbb{R}^{2} / \mathbb{Z}$ and $\Delta_{\mathbb{T}}=\Delta / \mathbb{Z}$ where the $\mathbb{Z}$-action on $\mathbb{R}^{2}$ is given by $(n,(a, b)) \mapsto(a+2 \pi n, b+2 \pi n)$ and $\Delta:=\left\{(a, b) \in \mathbb{R}^{2} \mid a=b\right\}$. One denotes the $\mathbb{Z}$-orbit of $(a, b) \in \mathbb{R}^{2}$ by $\langle a, b\rangle \in \mathbb{T}$. Note that $\mathbb{T}$ can be identified to $\mathbb{C} \backslash 0$ via the map $\langle a, b\rangle \mapsto z:=e^{(a-b) / 2+i(a+b) / 2}$. Via this identification, $\Delta_{\mathbb{T}}$ corresponds to the unit circle $\mathbb{S}^{1}=\{z \in \mathbb{C}:|z|=1\}$.

We will record the collections $\mathcal{B}_{r}^{c}(f) \sqcup \mathcal{B}_{r-1}^{o}(f)$ as a finite configuration of points in $\mathbb{T}=\mathbb{C} \backslash 0$, denoted by $C_{r}(f)$, and the collection $\mathcal{B}_{r}^{c o}(f) \sqcup \mathcal{B}_{r}^{o c}(f)$ as a finite configuration of points in $\mathbb{T} \backslash \Delta_{\mathbb{T}}=\mathbb{C} \backslash\left(0 \sqcup \mathbb{S}^{1}\right)$, denoted by $C_{r}^{m}(f)$.

More precisely, in the first case a closed $r$-bar code $[a, b]$ will be written as $\langle a, b\rangle \in$ $\mathbb{T}$ or the complex number $z=e^{(a-b) / 2+i(a+b) / 2} \in \mathbb{C} \backslash 0$ and an open $(r-1)$-bar code $(\alpha, \beta)$ as $\langle\beta, \alpha\rangle \in \mathbb{T}$ or the complex number $z=e^{(\beta-\alpha) / 2+i(\beta+\alpha) / 2} \in \mathbb{C} \backslash 0$. Similarly, in the second case, a closed-open $r$-bar code $[a, b)$ will be written as $\langle a, b\rangle \in \mathbb{T} \backslash \Delta_{\mathbb{T}}$ or the complex number $e^{(a-b) / 2+i(a+b) / 2} \in \mathbb{C} \backslash\left(0 \sqcup \mathbb{S}^{1}\right)$ and an open-closed $r$-bar code $(\alpha, \beta]$ as $\langle\beta, \alpha\rangle \in \mathbb{T} \backslash \Delta_{\mathbb{T}}$ or the complex number $e^{(\beta-\alpha) / 2+i(\beta+\alpha) / 2} \in \mathbb{C} \backslash\left(0 \sqcup \mathbb{S}^{1}\right)$.

In view of Theorem 1.1 a , if $f$ is in the homotopy class defined by $\xi \in H^{1}(X ; \mathbb{Z})$, then the configuration $C_{r}(f)$ has the total cardinality of the support ${ }^{4}$ exactly $\beta_{r}^{N}(X ; \xi)$ and can be regarded as a point in the $n$-fold symmetric product $S^{n}(\mathbb{T})$ of $\mathbb{T}$ where $n=\beta_{r}^{N}(X ; \xi)$. Identifying $\mathbb{T}$ with $\mathbb{C} \backslash 0$ as above, the space $S^{n}(\mathbb{T})$ identifies to the space of monic polynomials of degree $n$ with non-vanishing free coefficient, that is, $\mathbb{C}^{n-1} \times(\mathbb{C} \backslash 0)$, by assigning to a complex polynomial its configuration of zeros with multiplicities. Hence, each $C_{r}(f)$ can be regarded as a monic polynomial $P_{r}^{f}(z)$ of degree $n$ with non-vanishing free coefficient. We equip $S^{n}(\mathbb{T})$ with the topology of the symmetric product or equivalently with the topology of $\mathbb{C}^{n-1} \times(\mathbb{C} \backslash 0)$.

Let $C\left(X, \mathbb{S}^{1}\right)$ denote the space of all continuous maps equipped with the compact open topology and let $C_{\xi}\left(X, \mathbb{S}^{1}\right)$ be the connected component corresponding to $\xi$. Let $C_{\xi, t}\left(X, \mathbb{S}^{1}\right)$ be the subspace of tame maps in $C_{\xi}\left(X, \mathbb{S}^{1}\right)$. Our next result is the following theorem which will be referred to below as Strong Stability Theorem.

Theorem 1.2 (Stability). Suppose $X$ is a compact ANR. Then the assignment

$$
C_{\xi, t}\left(X, \mathbb{S}^{1}\right) \ni f \mapsto C_{r}(f) \in S^{n}(\mathbb{T}),
$$

equivalently,

$$
C_{\xi, t}\left(X, \mathbb{S}^{1}\right) \ni f \mapsto P_{r}^{f}(z) \in \mathbb{C}^{n-1} \times(\mathbb{C} \backslash 0),
$$

is continuous, where $n=\beta_{r}^{N}(X, \xi)$.

\footnotetext{
${ }^{4}$ The total cardinality of the support of a configuration is the sum of the multiplicities of its points.
} 
Moreover, if $X$ is homeomorphic to a simplicial complex, this extends to a continuous map, $C_{\xi}\left(X, \mathbb{S}^{1}\right) \rightarrow S^{n}(\mathbb{T})$, equivalently, $C_{\xi}\left(X, \mathbb{S}^{1}\right) \rightarrow \mathbb{C}^{n-1} \times(\mathbb{C} \backslash 0)$.

In particular, if $X$ is triangulable, then the configuration $C_{r}(f)$ and therefore the closed and open bar codes, can be defined for any continuous map. It is expected that the triangulability hypothesis can be removed. 5

The configuration $C_{r}(f)$, equivalently the polynomial $P_{r}^{f}(z)$, can be viewed as a refinement of the Novikov-Betti number in dimension $r$. The Poincaré duality for closed manifolds extends from Novikov-Betti numbers to these refinements and we have the following theorem.

Theorem 1.3 (Poincaré duality). If $M^{n}$ is a closed $\kappa$-orientable ${ }^{6}$ topological manifold and $f: M \rightarrow \mathbb{S}^{1}$ a tame map, then

$$
C_{r}(f)(\langle a, b\rangle)=C_{n-r}(f)(\langle b, a\rangle) .
$$

Equivalently, $C_{r}(f)(z)=C_{n-r}(f)(\tau(z))$ where $\tau(z):=1 / \bar{z}=z /|z|^{2}$ denotes the inversion across the unit circle, $z \in \mathbb{C} \backslash 0$.

The proofs of Theorems 1.2 and 1.3 use an alternative definition of the configuration $C_{r}(f)$. One defines the function $\delta_{r}^{f}$ on $\mathbb{T}$ with values in $\mathbb{N}_{0}$, with no reference to "bar codes" or to graph representations, and one verifies that it is equal to the configuration $C_{r}(f)$. One verifies Theorems 1.2 and 1.3 for $\delta_{r}^{f}$ instead of $C_{r}(f)$.

The Jordan blocks introduced in [3] via graph representations, can be also recovered in a different manner, more precisely, as the regular part of a linear relation. This makes their computations achievable by an algorithm less expensive than the one presented in [3], cf. [7.

A linear relation, $R: V \rightsquigarrow V$, is a concept generalizing a linear map, $V \rightarrow V$. To every linear relation $R$ on a $\kappa$-vector space $V$ one can associated canonically a pair, $R_{\text {reg }}=\left(V_{\text {reg }}, T_{\text {reg }}\right)$, where $V_{\text {reg }}$ is a $\kappa$-vector space and $T_{\text {reg }}: V_{\text {reg }} \rightarrow V_{\text {reg }}$ is a linear isomorphism. This construction will be discussed in Section 8.1

To a tame map $f: X \rightarrow \mathbb{S}^{1}$ one associates linear relations $R_{r}^{\theta}: H_{r}\left(f^{-1}(\theta)\right) \rightsquigarrow$ $H_{r}\left(f^{-1}(\theta)\right)$ described as follows. Let $\tilde{f}: \tilde{X} \rightarrow \mathbb{R}$ be the infinite cyclic covering defined by the pullback diagram

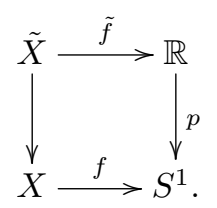

For $t$ with $p(t)=\theta$ the linear relation $R_{r}^{\theta}$ is obtained by passing to homology in the sequence, see Section 8.2 for more details,

$$
f^{-1}(\theta)=\tilde{f}^{-1}(t) \hookrightarrow \tilde{f}^{-1}([t, t+2 \pi]) \hookleftarrow \tilde{f}^{-1}(t+2 \pi)=f^{-1}(\theta) .
$$

We have the following result.

Theorem 1.4 (Monodromy theorem). If $f$ is a tame map and $r$ a non-negative integer, then for any angle $\theta$ the pair $\left(R_{r}^{\theta}\right)_{\mathrm{reg}}$ is isomorphic to

$$
\left(\bigoplus_{J \in \mathcal{J}_{r}(f)} V_{J}, \bigoplus_{J \in \mathcal{J}_{r}(f)} T_{J}\right)
$$

${ }^{5}$ Results on Hilbert cube manifolds permit to remove the triangulability hypothesis, cf. [5].

${ }^{6}$ If $\kappa$ has characteristic 2 any manifold is $\kappa$-orientable if not the manifold should be orientable. 
with $J=\left(V_{J}, T_{J}\right)$ and is a homotopy invariant of the pair $\left(X, \xi_{f}\right)$.

The next results refers to the collections $\mathcal{B}_{r}^{c o}(f)$ and $\mathcal{B}_{r}^{o c}(f)$ of mixed bar codes. First we note that the collection $\mathcal{B}_{r}^{c o}(f)$ can be identified to the collection of finite persistence intervals considered in [17] or [11] for the map $\tilde{f}: \tilde{X} \rightarrow \mathbb{R}$ made equivalent modulo $2 \pi$-translation. Similarly, the collection $\mathcal{B}_{r}^{o c}(f)$, after changing $(a, b]$ into $[-b,-a)$, can be identified to the collection of finite persistence intervals of the map $-\tilde{f}$ modulo $2 \pi$-translation.

The configurations $C_{r}^{m}(f)$ obtained by putting together $\mathcal{B}_{r}^{c o}(f)$ and $\mathcal{B}_{r}^{o c}(f)$ also enjoy a stability property and Poincaré duality, cf. Theorem 1.5 and Theorem 1.6 below, however with different quantitative and qualitative properties. Theorem 1.5 is a reformulation of the famous stability result of [1] and is stated here only for comparison with Theorem 1.2 .

Note that for tame angle valued maps in the same homotopy class the configurations $C_{r}^{m}(f)$ do not have the support of the same cardinality therefore a stability property will require a new topology on the set of configuration; in such topology the definition of "proximity" ignores the points near the diagonal $\Delta_{\mathbb{T}}$. This topology on the space of configurations of points in $\mathbb{T} \backslash \Delta_{\mathbb{T}}$, called the bottleneck topology, can be derived from a metric proposed in 11, the bottleneck metric.

Here is an alternative definition of the "bottleneck topology" on the set $\operatorname{Confg}(X \backslash$ $K$ ) of configurations of points in $X \backslash K, X$ locally compact space and $K$ a closed subset of $X$ without involving metric. Recall that a configuration is a map with finite support, $\delta: X \backslash K \rightarrow \mathbb{N}_{0}$. A base for the bottleneck topology is given by the collection of sets $\mathcal{U}(S)$ indexed by systems $S=\left\{\left(U_{1}, k_{1}\right), \ldots,\left(U_{r}, k_{r}\right), V\right\}$ satisfying

(i) $U_{i}, i=1, \ldots, r$ open subsets of $X \backslash K, V$ open neighborhood of $K$,

(ii) $k_{1}, k_{2}, \ldots, k_{r}$ positive integers.

and defined by

$$
\mathcal{U}(S):=\left\{\delta \in \operatorname{Confg}(X \backslash K) \mid \operatorname{supp}(\delta) \subset U_{1} \cup \cdots \cup U_{r} \cup V, \sum_{x \in U_{i}} \delta(x)=k_{i}\right\} .
$$

A set $\mathcal{U} \subset \operatorname{Confg}(X \backslash K)$ is open in the bottleneck topology if for any $\delta \in \mathcal{U}$ there exists $S$ such that

$$
\delta \in \mathcal{U}(S) \subset \mathcal{U} .
$$

When $X$ is a complete locally compact metric space and $K$ is a closed subspace the bottleneck metric of $\operatorname{Confg}(X \backslash K)$ given by the formulae in 11] induces the bottleneck topology described above.

The "main theorem" in [1] implies:

Theorem 1.5 (CEH stability). The assignment $f \mapsto C_{r}^{m}(f)$ is a continuous map from the space $C_{t}\left(X, \mathbb{S}^{1}\right)$ of tame maps to $\operatorname{Confg}\left(\mathbb{T} \backslash \Delta_{\mathbb{T}}\right)$ when the first space is equipped with the compact open topology and the second with the topology described above in case $(X, K)=\left(\mathbb{T}, \Delta_{\mathbb{T}}\right)$.

To better realize the differences between Theorem 1.2 and 1.5 we point out that:

(a) Arbitrary small perturbations of a tame map can introduce arbitrary many mixed bar codes, see Example 1 in Appendix B.

(b) Arbitrary small perturbations of some tame maps (which have closed $r$-bar codes of the form $[a, a]$ ) can decrease the number of closed $r$-barcodes and increase the number of open $(r-1)$-bar codes, see Example 2 in Appendix B. 
(c) Continuous deformations of tame maps can make the mixed $r$-barcodes, i.e., closed $r$-bar codes and open $r$-bar codes, appear or disappear. The decrease or the increase in the number of closed $r$-bar codes is at the expense of the increase or the decrease in the number of open $(r-1)$-barcodes, see Examples 2 and 3 in Appendix B.

(d) The assignment $f \mapsto C_{r}^{m}(f)$, as opposed to the assignment $f \mapsto C_{r}(f)$, can not be extended continuously to the entire space $C_{\xi}\left(X, \mathbb{S}^{1}\right)$ and this because of the lack of completeness of the bottleneck metric.

For the reader familiar with Morse theory we point out that the disappearance of a closed-open $r$-bar code by a continuous deformation of tame map is similar to the cancellation of a pair of two critical points one of index $r$ one of index $(r-1)$ in Morse theory as described in [27.

For a closed topological manifold $M^{n}$ the configurations $C_{r}^{m}(f)$ satisfy Poincaré duality but in analogy to the the Poincaré duality for the torsion subgroups of the integral homology groups for closed orientable manifolds. Precisely, we have the following result.

Theorem 1.6 (Poincaré duality). If $M^{n}$ is a closed $\kappa$-orientable topological manifold, $f: M \rightarrow \mathbb{S}^{1}$ a tame map, then

$$
C_{r}^{m}(f)(\langle a, b\rangle)=C_{n-1-r}^{m}(f)(\langle b, a\rangle) .
$$

Equivalently, $C_{r}^{m}(f)(z)=C_{n-1-r}^{m}(f)(\tau(z))$, where $\tau(z):=1 / \bar{z}=z /|z|^{2}$ denotes the inversion across the unit circle, $z \in \mathbb{C} \backslash 0$.

It is interesting to regard the elements (ii), (ii), (iii), that is, the critical values, bar codes and Jordan blocks associated to a tame angle valued map $f: X \rightarrow \mathbb{S}^{1}$, as parallels to the rest points, the isolated trajectories between rest points and the closed trajectories (actually Poincaré return maps for closed trajectories) of a vector field which has a Morse angle-valued map $f: M \rightarrow \mathbb{S}^{1}$ as Lyapunov map. These last ones are the concepts which enter the Morse-Novikov theory, cf. 30, 31, and are related to the topology of $\left(X, \xi_{f}\right)$, where $\xi_{f}$ denotes the integral cohomology class defined by $f$, in a similar way as the elements described in (ii), (iii) and (iii) are.

The last result, Theorem 1.7 below, improves on Morse inequalities for realvalued maps resp. Morse-Novikov inequalities for angle-valued maps, the simplest and most familiar applications of Morse resp. Morse-Novikov theory, cf. [26, 31].

Recall that for a smooth closed manifold $M^{n}$ a smooth real or angle valued map is Morse if all critical points $x$ are non degenerate, hence have a Morse index, $\operatorname{ind}_{f}(x) \in\{0,1, \ldots, n\}$. Recall that a point $x \in M$ is critical if, with respect to any local coordinates $\left(t_{1}, t_{2}, \ldots, t_{n}\right)$ with $x$ given by $t_{1}=t_{2}=\cdots=t_{n}=0$, all partial derivatives $\partial f / \partial t_{i}(0)$ vanish. A critical point is non-degenerate if in addition the Hessian, i.e. the symmetric matrix $\frac{\partial^{2} f}{\partial t_{i} \partial t_{j}}(0)$, has all eigenvalues nonzero. These eigenvalues are all real, and the Morse index of $f$ at $x$ coincides with the number of negative eigenvalues. The concepts critical points, non-degenerate critical points, and index of a non-degenerate critical points are independent of the local coordinates $\left(t_{1}, t_{2}, \ldots, t_{n}\right)$. 
Let $c_{i}(f)$ be the number of critical points of index $i$. The Morse inequalites claim that for any $r \in \mathbb{N}_{0}$, and with respect to any field $\kappa$, one has

$$
\sum_{i=0}^{r}(-1)^{r-i} c_{i}(f) \geq \sum_{i=0}^{r}(-1)^{r-i} b_{i}
$$

where $b_{i}=\beta_{i}(M)$ if $f$ is real-valued Morse map, and $b_{i}=\beta_{i}^{N}\left(M ; \xi_{f}\right)$ if $f$ is an angle-valued Morse map, see [26, Equation $\left.\left(4_{\lambda}\right)\right]$.

The following result refines the Morse inequalities:

Theorem 1.7. Let $M^{n}$ be closed smooth manifold, 7 consider a field $\kappa$, and suppose $r$ is a non-negative integer. If $f: M \rightarrow \mathbb{R}$ is a real-valued Morse map, then

$$
c_{r}(f)=\beta_{r}(M)+\sharp \mathcal{B}_{r}^{c o}(f)+\sharp \mathcal{B}_{r-1}^{c o}(f) .
$$

Moreover, if $f: M \rightarrow \mathbb{S}^{1}$ is an angle-valued Morse map, then

$$
c_{r}(f)=\beta_{r}^{N}\left(M ; \xi_{f}\right)+\sharp \mathcal{B}_{r}^{c o}(f)+\sharp \mathcal{B}_{r-1}^{c o}(f) .
$$

Note that the right side of the above equalities make sense for an arbitrary compact ANR equipped with a tame map rather than compact manifolds equipped with a Morse function, an attractive feature in comparison with the classical MorseNovikov theory.

The paper contains, in addition to the present section which summarizes the results, eight more sections which describe the concepts involved in and provide the proofs of the results and three appendices. In Section 2 we review simple results about graph representations of the two graphs relevant for this paper, $G_{2 m}$ and $\mathcal{Z}$. In Section 3 we define the sets $\mathcal{B}_{r} \cdots(f)$ and $\mathcal{J}_{r}(f)$ and provide the preliminaries for the proof of Theorem 1.1. In Section 4 we prove Theorem 1.1. In Section 5 we define the function $\delta_{r}^{f}$ and prove Theorem 1.2. In Sections 6 and 7 we discuss the Poincaré duality for the configurations $C_{r}(f)$ and $C_{r}^{m}(f)$ and prove Theorems 1.3 and 1.6. In Section 8 we discuss some linear algebra of linear relations and prove Theorem 1.4. In Section 9 we verify Theorem 1.7. Appendix A provides an example of tame map and describes its bar codes and Jordan cells. Appendix B illustrates the behavior of bar codes with respect to a continuous deformation of the map. Appendix C provides a few observations about $\kappa\left[t^{-1}, t\right]$-modules.

Note that if $f: X \rightarrow \mathbb{S}^{1}$ is not surjective, then the set $\mathcal{J}_{r}(f)$ vanishes for all $r$. Note also that a real-valued $f$ can be viewed as a non-surjective angle-valued map, and the bar codes are essentially the same as the zigzag persistence barcodes cf. [10. In this case there is no need to consider $\mathbb{T}$ and $\mathbb{T} \backslash \Delta_{\mathbb{T}}$; the natural place for the support of the configuration $C_{r}(f)$, consisting of closed $r$-barcodes and open $(r-1)$-barcodes, is $\mathbb{C}$; and the natural place for the support of $C_{f}^{m}(f)$, consisting of the closed-open $r$-barcodes and open-closed $r$-barcodes, is $\mathbb{C} \backslash \Delta$.

Prior work. Relating the topology of a space to the homological behavior of the sublevel sets of a real or angle-valued map represents what "persistence theory" introduced in [17] intends to do. Prior efforts to extend Morse theory to all continuous real-valued functions (fonctionelles) can be found in the papers of M. Morse [28] and R. Deheuvels [13], which preceded persistence theory. The work of R. Deheuvels [13, permits to derive the barcodes (the support of persistence diagrams as

\footnotetext{
${ }^{7}$ The result remains true for compact manifolds with boundary satisfying an appropriate hypothesis on the behavior of $f$ along the boundary.
} 
considered in [17] or [16]) from the differentials of Leray spectral sequence of a real valued tame map. The same observation holds about the barcodes in the zigzag persistence and persistence for circle valued maps but we can not find this in this existing in literature.

A stability phenomena for the persistence diagrams associated to a real valued map in classical persistence theory was first established in [11.

The first use of graph representations in connection with persistence appears first in [10] under the name of zigzag persistence. The graphs considered are all linear finite graphs whose collection of indecomposable representations is finite and not hard to describe and interpret as bar codes (of four types).

The definition of bar codes and of Jordan cells for $\mathbb{S}^{1}$-valued tame maps was first provided in [3] based on graph representations of the cyclic graph $G_{2 m}$ whose indecomposable representations are more complex and led in addition to bar codes to Jordan cells.

The referee points out a that a number of the results in this paper are reminiscent of behavior of the bar codes in zigzag persistence cf. [10] and this deserves to be mentioned. We are happy to do so. Reminiscences of the work of 12 in the Poincaré duality Theorems 1.6 should be also acknowledged.

Some more recent work. Using results from topology of Hilbert cube manifolds, it was recently observed that the hypothesis " $X$ homeomorphic to a simplicial complex" in Theorem 1.2 can be weaken to " $X$ compact ANR", and the hypothesis "tame map" in Theorems 1.1. 1.3, and 1.4 can be weaken to "continuous map" cf [5] and [6].

In case of a real valued map and in the presence of a scalar product on $H_{r}(X)$ (the field $\kappa$ being $\mathbb{R}$ or $\mathbb{C}$ ) the configuration $C_{r}(f)$ can be implemented as a configuration $\hat{\delta}_{r}^{f}$ of subspaces $\hat{\delta}_{r}^{f}(z) \subseteq H_{r}(X), z$ in the support of $C_{r}(f)$, which are mutually orthogonal and have $\operatorname{dim} \hat{\delta}_{r}^{f}(z)$ equal to the multiplicity of $z$. The assignment $f \rightsquigarrow$ $\hat{\delta}_{r}^{f}$ remains continuous w.r. to the obvious topologies and in case of closed manifolds Poincaré duality between configurations $C_{r}(f)$ extends to the configurations $\hat{\delta}_{r}^{f}$ of vector spaces. This is the case when $X$ is the underlying space of a closed Riemannian manifold $M^{n}$ and $\kappa=\mathbb{R}$ with the scalar product on $H_{r}(M)$ provided by the identification with the space of harmonic forms in complementary dimension $(n-r)$. This will be discussed in details in [5].

A similar fact remains true for angle valued maps. If $\kappa=\mathbb{C}$ the Novikov homology $H_{r}^{N}\left(X ; \xi_{f}\right)$ can be replaced by the $L_{2}$-homology $H_{r}^{L_{2}}(\tilde{M})$ of the infinite cyclic cover $\tilde{X}$ defined by the map $f$. When regarded as a Hilbert module over the von Neumann algebra $L^{\infty}\left(\mathbb{S}^{1}\right)$ this Hilbert module has the von Neumann dimension equal to the Novikov-Betti number $\beta_{r}^{N}\left(X ; \xi_{f}\right)$. The mutually orthogonal subspaces are in this case mutually orthogonal Hilbert submodules. This will be discussed in [6.

If $f: M^{n} \rightarrow \mathbb{R}$ or $f: M^{n} \rightarrow \mathbb{S}^{1}$ is a Morse function, Lyapunov for a smooth vector field $X$ on a closed manifold $M$, the Morse complex resp. the Novikov complex tensored by a field $\kappa$ derived geometrically from the critical points of $f$ and the isolated trajectories of $X$ between critical points, can be recovered up to isomorphism from the closed, open and closed-open bar codes of $f$ via the results discussed in this paper. Actually the closed-open barcodes determine the rank of the boundary maps in these complexes. More about can be found in the forthcoming book [8]. 
The precise relation between Reidemeister torsion, closed trajectories for a vector field with an angle valued map $f$ as Lyapunov and the Jordan cells and barcodes is the topic of work in preparation under the name Alternative to Morse-Novikov theory.

Applications. The angle valued maps are as interesting and frequent as the real valued maps. Observing/sampling an environment/shape from a central point in each direction should be as interesting and natural as observing the sublevel sets with respect to a real valued function of a shape.

So far there are pleasant mathematical applications of the results in this paper and of the subsequent work, cf. [5, 6, 7, 8, in Computational Topology, Geometric Analysis and Dynamics.

- Computational Topology. Theorems 1.1 and 1.4 imply precise relations between Betti numbers of $X$, Novikov Betti numbers and the monodromy, i.e., Jordan cells of a pair $\left(X ; \xi_{f}\right)$. They lead to computer implementable algorithms for the calculation of the last two without involving the infinite cyclic cover of $\xi_{f}$, a computer unfriendly object (being infinite even when $X$ is a finite simplicial complex), cf. [3] and [7. In particular, they lead to alternative methods to calculate the Alexander polynomial of knots and some Reidemeister torsions, to recognition of when $f: X \rightarrow \mathbb{S}^{1}$ is homotopic to a fibration with compact fiber, and in this case to the calculation of the Betti numbers of the fiber. A paper on these type of results is in preparation.

- Algebraic Topology of complements of complex hyper surfaces. The complement of a complex hyper surface in $\mathbb{C}^{n}$ comes equipped with a natural angle valued map. The relevant algebraic topology invariants of this space are quite important in algebraic geometry. They can be express in therms of bar codes and Jordan cells and then are in principle computable. Results in this direction are available in 6, 7, 8.

- Geometric Analysis. The implementation of $C_{r}(f)$ to a configuration of mutually orthogonal spaces provides an orthogonal decomposition of the space of complex harmonic $(n-r)$-differential forms in subspaces, i.e., components, each subspace corresponding to the complex number represented by a closed $r$-barcode or an open $(r-1)$-barcode. For generic $f$ each component has dimension one. A pleasant consequences of this additional structure is the existence for a generic pair $(g, f) g$-Riemannian metric, $f$ smooth map of a canonical base in the space of $r$-differential forms, analogous of the base provided by the trigonometric functions in the space of smooth functions on $\mathbb{S}^{1}$.

- Dynamics. The presence of Jordan cells (i.e. non-trivial monodromy) for a map $f: X \rightarrow \mathbb{S}^{1}$ implies the existence of closed trajectories for flows on $X$ for which $f: X \rightarrow \mathbb{S}^{1}$ is Lyapunov. The non-triviality of $C_{r}^{m}(f)$ implies existence of instantons between rest points. More precisely, Theorem 1.7 above permits to describe the rank of boundary map $\partial_{r}$ in the Morse complex or the Novikov complex relevant quantity in the counting of instantons. More can be found in Chapter 8 of the book in preparation [8].

The authors thank the referee for a careful reading and useful observations. The present version ows much to his critics and suggestions. 


\section{GRAPH REPRESEntations}

Fix a field $\kappa$. Let $\Gamma$ be an oriented graph, possibly with infinitely many vertices. A $\Gamma$-representation $\rho$ assigns to each vertex $x$ of $\Gamma$ a finite dimensional vector space $V_{x}$ and to each arrow $a: x \rightarrow y$ between two vertices $x$ and $y$ a linear map $\phi_{a}: V_{x} \rightarrow V_{y}$. Suppose $\rho^{\prime}$ is another $\Gamma$-representation with vector spaces $V_{x}^{\prime}$ and linear maps $\phi_{a}^{\prime}: V_{x}^{\prime} \rightarrow V_{y}^{\prime}$. A morphism from $\rho$ to $\rho^{\prime}$ is a collection of linear maps $\psi_{x}: V_{x} \rightarrow V_{x}^{\prime}$ such that $\phi_{a}^{\prime} \psi_{x}=\psi_{y} \phi_{a}$ for all arrows $a: x \rightarrow y$ between any two vertices $x$ and $y$. More succinctly, a $\Gamma$-representation may be defined as a covariant functor from the (small) category generated by the graph $\Gamma$ to the (abelian) category of finite dimensional vector spaces. A morphism of $\Gamma$-representations is just a natural transformation between two such functors. Consequently, $\Gamma$-representations and morphisms between $\Gamma$-representations form an abelian category, see [2, 32. In particular, the concepts of isomorphism (equivalence), direct sum, kernel, image, and short exact sequence are well defined for (morphisms between) $\Gamma$-representations.

Suppose $\rho_{\alpha}, \alpha \in \mathcal{A}$, is a family of $\Gamma$-representations with vector spaces $V_{x}^{\alpha}$ and linear maps $\phi_{a}^{\alpha}: V_{x}^{\alpha} \rightarrow V_{y}^{\alpha}$. If, for every vertex $x$, all but finitely many of the vector spaces $V_{x}^{\alpha}$ are trivial, then one considers the $\Gamma$-representation $\bigoplus_{\alpha \in \mathcal{A}} \rho_{\alpha}$ which assigns to a vertex $x$ the vector space $\bigoplus_{\alpha} V_{x}^{\alpha}$ and to an arrow $a: x \rightarrow y$ the linear map $\bigoplus_{\alpha} \phi_{a}^{\alpha}: \bigoplus_{\alpha} V_{x}^{\alpha} \rightarrow \bigoplus_{\alpha} V_{y}^{\alpha}$.

A $\Gamma$-representation $\rho$ is called: regular, if all the linear maps $\phi_{a}$ are isomorphisms; with finite support, if $V_{x}=0$ for all but finitely many vertices; and indecomposable, if it is not isomorphic to the sum of two non-trivial representations.

A standard result in abelian categories, see [1, Theorem 1], [32, Chapter 5] or 2. Theorem 6.45], formulated for $\Gamma$-representations with finite support, reads:

Theorem 2.1 (Krull-Remak-Schmidt). Any $\Gamma$-representation with finite support is isomorphic to a direct sum $\rho_{1} \oplus \cdots \oplus \rho_{n}$ with indecomposable summands $\rho_{i}$. Moreover, the components $\rho_{i}$ are unique up to isomorphism and reordering.

In this paper the oriented graph $\Gamma$ of primary concern will be $G_{2 m}$ and for technical reasons we will need the infinite oriented graph $\mathcal{Z}$. The graph $\Gamma=G_{2 m}$ has vertices $x_{1}, x_{2}, \ldots, x_{2 m}$ and arrows $a_{i}: x_{2 i-1} \rightarrow x_{2 i}, 1 \leq i \leq m$, and $b_{i}: x_{2 i+1} \rightarrow$ $x_{2 i}, 1 \leq i \leq m-1$ and $b_{m}: x_{1} \rightarrow x_{2 m}$, see Figure 11. The graph $\Gamma=\mathcal{Z}$ has vertices $x_{i}, i \in \mathbb{Z}$, and arrows $a_{i}: x_{2 i-1} \rightarrow x_{2 i}$ and $b_{i}: x_{2 i+1} \rightarrow x_{2 i}$, see Figure 2 .

Both $G_{2 m}$ and $\mathcal{Z}$-representations $\rho$ will be recorded as

$$
\rho=\left\{V_{r}, \alpha_{i}: V_{2 i-1} \rightarrow V_{2 i}, \beta_{i}: V_{2 i+1} \rightarrow V_{2 i}\right\}
$$

in the first case with $1 \leq r \leq 2 m, 1 \leq i \leq m$, with the convention that $V_{2 m+1}=V_{1}$, in the second case with $r, i \in \mathbb{Z}$.

Any regular $G_{2 m}$-representation $\rho=\left\{V_{r}, \alpha_{i}, \beta_{i}\right\}$, not necessarily indecomposable, is equivalent i.e. isomorphic to the representation

$$
\rho(V, T):=\left\{V_{r}^{\prime}=V, \alpha_{1}^{\prime}=T, \alpha_{i}^{\prime}=\operatorname{Id} i \neq 1, \beta_{i}^{\prime}=\operatorname{Id}\right\}
$$

with $T=\beta_{m}^{-1} \cdot \alpha_{m}^{-1} \cdots \beta_{1}^{-1} \cdot \alpha_{1}$. The isomorphism i.e. conjugacy class of the pair $(V, T)$ is called monodromy.

According to the Krull-Remak-Schmidt theorem, every $G_{2 m}$-representation $\rho$ decomposes as sum, $\rho \cong \rho^{\prime} \oplus \rho^{\prime \prime}$, where $\rho^{\prime \prime}$ is regular and $\rho^{\prime}$ has no non-trivial regular summand. Moreover, both parts $\rho^{\prime}$ and $\rho^{\prime \prime}$ are unique up to isomorphisms. The regular part $\rho^{\prime \prime}$ provides the monodromy of $\rho$ which as pointed out above is determined by an isomorphism class of pairs $(V, T)$. 


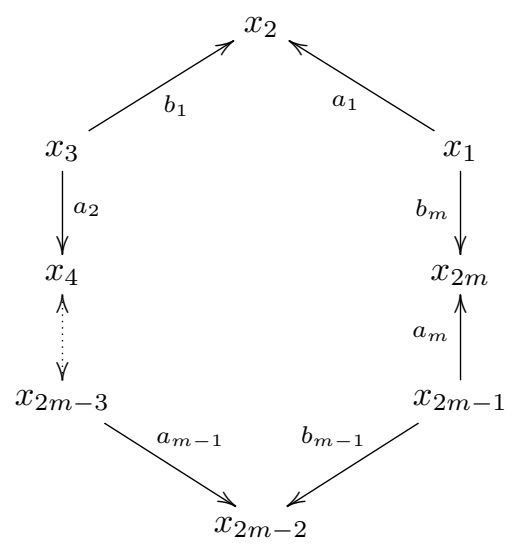

Figure 1. The graph $G_{2 m}$.

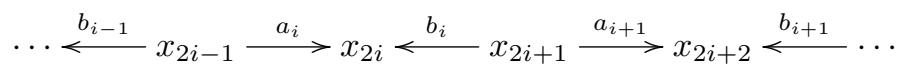

FiguRE 2. The graph $\mathcal{Z}$.

The $\mathcal{Z}$-representations we consider are either with finite support or periodic. The representation is periodic if for some integer $N, V_{r}=V_{r+2 N}, \alpha_{i}=\alpha_{i+N}, \beta_{i}=\beta_{i+N}$. Both type of $\mathcal{Z}$-representations, periodic and with finite support, as well as a finite direct sum of of such representations will be referred to as good $\mathcal{Z}$-representations.

2.1. The indecomposable $G_{2 m}$-representations and the indecomposable good $\mathcal{Z}$-representations. The indecomposable $G_{2 m}$-representations are of two types, cf. [3, Section 4]. In a slightly different formulation the identification below was first established in [29] and [15].

Type I (bar codes). These representations are labeled by the four types of intervals with integer valued ends $r$ and $s, r \leq s, 1 \leq r \leq m$, namely $[r, s]$ with $r \leq s$, and $(r, s),[r, s),(r, s]$ with $r<s$. If $I$ is an interval of this form, then the corresponding representation will be denoted by $\rho^{G}(I)$. More explicitly, they are denoted by $\rho^{G}(\{r, s\})$ with "\{" notation for either "[" or "(" and "\}" for either "]" or ")" and graphically described as follows ${ }^{8}$

Suppose the vertices $x_{1}, x_{2}, \ldots, x_{2 m-1}, x_{2 m}$ are located counter-clockwise on the unit circle, say at the the angles $t_{1}<\theta_{1}<t_{2}<\theta_{2}<\cdots<t_{m}<\theta_{m}$, with $0<t_{1}$ and $\theta_{m} \leq 2 \pi$.

To describe the representation $\rho^{G}(\{i, j+m k\}), 1 \leq i, j \leq m$, draw the counterclockwise spiral curve from $a=\theta_{i}$ to $b=\theta_{j}+2 \pi k$ with the ends a black or an empty circle to indicate "closed" or "open" interval. Black circle indicates that the end is on the spiral, empty circle that is not.

\footnotetext{
${ }^{8} \mathrm{~A}$ simpler labeling is possible but the one proposed is consistent with the geometric situation the representations are derived from.
} 


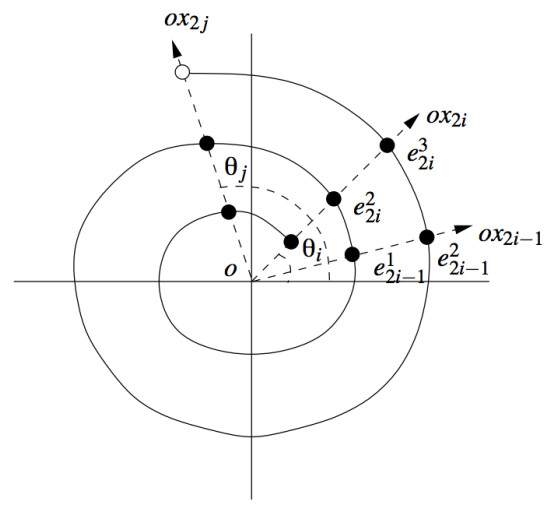

Figure 3 . The spiral for $[i, j+2 m)$.

The vector space $V_{i}$ is generated by the intersection points of the spiral with the radius corresponding to the vertex $x_{i}$ and $\alpha_{i}$ and $\beta_{i}$ are defined on generators as follows: A generator $e$ of $V_{2 i \pm 1}$ is sent to the generator $e^{\prime}$ of $V_{2 i}$ if connected by a piece of spiral or to 0 if not. The spiral in Figure 3 below corresponds to $k=2$, and defines the representation $\rho^{G}([i, j+2 m))$.

Type II (Jordan blocks / cells). They are labeled by Jordan blocks $J=(V, T)$ and denoted by $\rho^{G}(J)$. Recall that a Jordan block is an isomorphism class of indecomposable pairs $(V, T), V$ a vector space $T: V \rightarrow V$ an isomorphism. The representation $\rho^{G}(J)$ has all vector spaces equal to $V, \alpha_{1}=T$ and $\beta_{1}=\alpha_{i}=\beta_{i}=$ Id for $2 \leq i \leq m$. If $J=\left(\kappa^{k}, T(\lambda, k)\right)$ we also write $\rho^{G}(J):=\rho^{G}(\lambda, k)$.

One refers to both the labeling interval $\{r, s\}$ and the representation $\rho^{G}(\{r, s\})$ as bar code and to the indecomposable pair $J$ and the representation $\rho^{G}(J)$ as Jordan block.

By the Krull-Remak-Schmidt theorem and the classification of indecomposables, any $G_{2 m}$-representation $\rho$ can be decomposed as a sum of indecomposables,

$$
\rho \cong \bigoplus_{I \in \mathcal{B}(\rho)} \rho^{G}(I) \oplus \bigoplus_{J \in \mathcal{J}(\rho)} \rho^{G}(J) .
$$

Here $\mathcal{B}(\rho)$ denotes the collection of all bar codes (with proper multiplicity) appearing in the decomposition of $\rho$, and $\mathcal{J}(\rho)$ denotes the collection of all Jordan blocks (with proper multiplicity) appearing in the decomposition of $\rho$.

We further decompose,

$$
\mathcal{B}(\rho)=\mathcal{B}^{c}(\rho) \sqcup \mathcal{B}^{o}(\rho) \sqcup \mathcal{B}^{c o}(\rho) \sqcup \mathcal{B}^{o c}(\rho)
$$

where $\mathcal{B}^{c}(\rho), \mathcal{B}^{o}(\rho), \mathcal{B}^{c o}(\rho)$ and $\mathcal{B}^{o c}(\rho)$ denote the subcollections (with multiplicities) of barcodes with both ends closed, open, closed-open and open-closed, respectively. For $\lambda \in \kappa \backslash 0$ one denotes by $\mathcal{J}_{\lambda}(\rho)$ the collection (with multiplicities) of Jordan blocks with eigenvalue $\lambda !^{9}$

\footnotetext{
${ }^{9}$ If the linear map $T$ in the Jordan block $J=(V, T)$ has an eigenvalue $\lambda \in \kappa$ then this is the only eigenvalue, and $J$ is similar to $\left(\kappa^{k}, T(\lambda, k)\right)$, see 11 .
} 
The indecomposable $\mathcal{Z}$-representations with finite support are all bar codes indexed by four type of intervals $I$ with ends $i$ and $j,[i, j]$ with $i \leq j$, or $[i, j),(i, j]$, $(i, j)$ with $i<j$ and denoted by $\rho^{\mathcal{Z}}(I)$. The only periodic indecomposable representation is denoted by $\rho_{\infty}^{\mathcal{Z}}$. The representation denoted by $\rho^{\mathcal{Z}}(I)$ has all vector spaces equal to either $\kappa$ or 0 , the linear maps $\alpha_{i}$ and $\beta_{j}$ are equal to the identity if both, the source and the target, are non-trivial and zero otherwise. Precisely,

(i) $\rho^{\mathcal{Z}}([i, j]), i \leq j$ has $V_{r}=\kappa$ for $r=2 i, \ldots, 2 j$, and $V_{r}=0$ otherwise,

(ii) $\rho^{\mathcal{Z}}([i, j)), i<j$ has $V_{r}=\kappa$ for $r=2 i, \ldots, 2 j-1$, and $V_{r}=0$ otherwise,

(iii) $\rho^{\mathcal{Z}}((i, j]), i<j$ has $V_{r}=\kappa$ for $r=2 i+1, \ldots, 2 j$, and $V_{r}=0$ otherwise,

(iv) $\rho^{\mathcal{Z}}((i, j)), i<j$ has $V_{r}=\kappa$ for $r=2 i+1, \ldots, 2 j-1$, and $V_{r}=0$ otherwise.

Both, the labeling interval $I$ and the representation $\rho^{\mathcal{Z}}(I)$, will be referred to as bar code.

The indecomposable representation $\rho_{\infty}^{\mathcal{Z}}$, has all vector spaces $V_{r}=\kappa$ and all linear maps $\alpha_{i}=\beta_{i}=\mathrm{Id}$.

The Krull-Remak-Schmidt decomposition for representations with finite support extends to all good $\mathcal{Z}$-representations. For the reader's convenience an argument is presented at the end of the next section since it involves the definition of truncation.

Precisely, any such (good) representation $\rho$ is a sum (in the sense described above) of possibly infinitely many indecomposables with finite support and finitely many copies of $\rho_{\infty}^{\mathcal{Z}}$,

$$
\rho \cong \bigoplus_{I \in \mathcal{B}(\rho)} \rho^{\mathcal{Z}}(I) \oplus \bigoplus_{n} \rho_{\infty}^{\mathcal{Z}},
$$

with indecomposable factors and their multiplicity unique up to isomorphism. Here $\mathcal{B}(\rho)$ the collection of all bar codes (with multiplicity) appearing in the decomposition, and $\bigoplus_{n} \rho_{\infty}^{\mathcal{Z}}$ denotes the sum of $n$ copies of $\rho_{\infty}^{\mathcal{Z}}$. Each indecomposable $\rho^{\mathcal{Z}}(I)$ or $\rho_{\infty}^{\mathcal{Z}}$ appears with finite multiplicity. We let $\mathcal{B}^{c}(\rho), \mathcal{B}^{o}(\rho), \mathcal{B}^{c o}(\rho)$ and $\mathcal{B}^{o c}(\rho)$ denote the subcollections (with multiplicities) of closed, open, closed-open and open-closed bar codes in $\mathcal{B}(\rho)$. Moreover, $\mathcal{J}^{\mathcal{Z}}(\rho)$ denotes the collection of all copies of $\rho_{\infty}^{\mathcal{Z}}$ which appear as independent direct summands in $\rho$. The decomposition $(3)$ is discussed in the next subsection.

In view of the above comments, statements about $G_{2 m}$-representations or about good $\mathcal{Z}$-representations, formulated in this paper, will be verified first for the indecomposable representations described above and if hold true, by the Krull-RemakSchmidt decomposition theorem, concluded for arbitrary representations.

2.2. Two basic constructions. The infinite cyclic covering of a $G_{2 m}$-representation $\rho=\left\{V_{r}, a_{i}, b_{i}, 1 \leq r \leq 2 m, 1 \leq i \leq m\right\}$ is the periodic $\mathcal{Z}$-representation $\tilde{\rho}:=\left\{\tilde{V}_{r}, \tilde{a}_{i}, \tilde{b}_{i}, r, i \in \mathbb{Z}\right\}$ defined by $\tilde{V}_{r+2 m k}=V_{r}, \tilde{a}_{i+k m}=a_{i}$, and $\tilde{b}_{i+k m}=b_{i}$. When applied to indecomposable $\rho^{G}(I)$ or $\rho^{G}(J)$, where $I$ denotes an interval and $J=(V, T)$ is a Jordan block, one obtains:

$$
\begin{aligned}
& \widetilde{\rho^{G}(I)}=\bigoplus_{k \in \mathbb{Z}} \rho^{\mathcal{Z}}(I+m k) \\
& \widetilde{\rho^{G}(J)}=\bigoplus_{n} \rho_{\infty}^{\mathcal{Z}}, \quad n=\operatorname{dim} V, \quad J=(V, T) .
\end{aligned}
$$

Here $I+r, r \in \mathbb{Z}$ denotes the translate of the interval $I$, by $r$ units.

The truncation $T_{k, l}(\rho)$ of a $\mathcal{Z}$-representation $\rho$ is defined for any pair of integers $k, l$ with $k \leq l$. If $\rho$ is a $G_{2 m}$-representation, then the truncation $T_{k, l}(\rho)$ is defined 
for any pair of integers $k, l$ with $1 \leq k \leq l \leq m$. In either case, if $\rho=\left\{V_{r}, \alpha_{i}, \beta_{i}\right\}$ is a representation then the truncation is defined by $T_{k, l}(\rho):=\left\{V_{r}^{\prime}, \alpha_{i}^{\prime}, \beta_{i}^{\prime}\right\}$ where:

$$
\begin{aligned}
V_{r}^{\prime} & = \begin{cases}V_{r} & \text { if } 2 k \leq r \leq 2 l, \text { and } \\
0 & \text { otherwise }\end{cases} \\
\alpha_{r}^{\prime} & = \begin{cases}\alpha_{r} & \text { if } k+1 \leq r \leq l, \text { and } \\
0 & \text { otherwise }\end{cases} \\
\beta_{r}^{\prime} & = \begin{cases}\beta_{r} & \text { if } k \leq r \leq l-1, \text { and } \\
0 & \text { otherwise }\end{cases}
\end{aligned}
$$

When applied to indecomposable $\mathcal{Z}$-representations one obtains

$$
\begin{aligned}
T_{k, l}\left(\rho_{\infty}^{\mathcal{Z}}\right) & =\rho^{\mathcal{Z}}([k, l]), \\
T_{k, l}\left(\rho^{\mathcal{Z}}(I)\right) & =\rho^{\mathcal{Z}}(I \cap[k, l]),
\end{aligned}
$$

and when applied to indecomposable $G_{2 m}$-representations one obtains

$$
\begin{array}{ll}
T_{k, l}\left(\rho^{G}(I)\right)=\bigoplus_{r \in \mathbb{Z}} \rho^{G}\left(I_{r}\right), \quad I_{r}=(I+r m) \cap[k, l], \\
T_{k, l}\left(\rho^{G}(J)\right)=\bigoplus_{n} \rho^{G}([k, l]), \quad n=\operatorname{dim} V, \quad J=(V, T) .
\end{array}
$$

Here $I+r m$ denotes the translate of the interval $I$ to the right by $r m$ units.

Given a $G_{2 m}$-representation $\rho$ one writes: $\tilde{\mathcal{J}}(\rho)$ for the collection which contains with any Jordan block $J=(V, T) \in \mathcal{J}(\rho)$, a number of $n(J)=\operatorname{dim}(V)$ copies of $\rho_{\infty}^{\mathcal{Z}}$ hence a total of $\sum_{J=(V, T) \in \mathcal{J}(\rho)} \operatorname{dim} V$ copies of $\rho_{\infty}^{\mathcal{Z}}$, and $\tilde{\mathcal{B}}^{-}(\rho):=\{I+2 \pi k \mid$ $\left.I \in \mathcal{B}^{-}(\rho), k \in \mathbb{Z}\right\}$ with $\tilde{\mathcal{B}}^{-}$any of $\tilde{\mathcal{B}}, \tilde{\mathcal{B}}^{c}, \tilde{\mathcal{B}}^{o}, \tilde{\mathcal{B}}^{c o}, \tilde{\mathcal{B}}^{o c}$.

In terms of this notation is convenient to keep in mind the following bookkeeping.

Lemma 2.2. (a) If $\rho$ is a $G_{2 m}$-representation then

$$
\begin{gathered}
\mathcal{B}(\tilde{\rho})=\tilde{\mathcal{B}}(\rho), \quad \mathcal{J}(\tilde{\rho})=\tilde{\mathcal{J}}(\rho), \\
\mathcal{B}^{c}(\tilde{\rho})=\tilde{\mathcal{B}}^{c}(\rho), \quad \mathcal{B}^{o}(\tilde{\rho})=\tilde{\mathcal{B}}^{o}(\rho), \quad \mathcal{B}^{c o}(\tilde{\rho})=\tilde{\mathcal{B}}^{c o}(\rho), \quad \mathcal{B}^{o c}(\tilde{\rho})=\tilde{\mathcal{B}}^{o c}(\rho),
\end{gathered}
$$

and:

$\mathcal{B}^{c}\left(T_{k, l}(\rho)\right)=\{I \cap[k, l]: I \in \tilde{\mathcal{B}}(\rho)$ such that $I \cap[k, l]$ is non-empty and closed $\}$

$\sqcup\{[k, l]$ with multiplicity $\sharp \tilde{\mathcal{J}}(\rho)\}$,

$\mathcal{B}^{o}\left(T_{k, l}(\rho)\right)=\left\{I \in \tilde{\mathcal{B}}^{o}(\rho): I \subset[k, l]\right\}$

$\mathcal{J}\left(T_{k, l}(\rho)\right)=\emptyset$.

(b) If $\rho$ is a good $\mathcal{Z}$-representation then:

$\mathcal{B}^{c}\left(T_{k, l}(\rho)\right)=\{I \cap[k, l]: I \in \mathcal{B}(\rho)$ such that $I \cap[k, l]$ is non-empty and closed $\}$

$\sqcup\{[k, l]$ with multiplicity $\sharp \mathcal{J}(\rho)\}$,

$\mathcal{B}^{o}\left(T_{k, l}(\rho)\right)=\left\{I \in \mathcal{B}^{o}(\rho): I \subset[k, l]\right\}$,

$\mathcal{J}\left(T_{k, l}(\rho)\right)=\emptyset$. 
Proof. To see (a) observe that infinite cyclic covering and truncation are both additive constructions, that is to say, $\widetilde{\rho_{1} \oplus \rho_{2}}=\tilde{\rho}_{1} \oplus \tilde{\rho}_{2}$ and $T_{k, l}\left(\rho_{1} \oplus \rho_{2}\right)=$ $T_{k, l}\left(\rho_{1}\right) \oplus T_{k, l}\left(\rho_{2}\right)$ for any two $G_{2 m}$-representations $\rho_{1}$ and $\rho_{2}$. The expressions for $\mathcal{B}(\rho), \mathcal{B}^{c}(\rho), \mathcal{B}^{o}(\rho), \mathcal{B}^{c o}(\rho), \mathcal{B}^{o c}(\rho)$, and $\mathcal{J}(\rho)$ thus follow immediately from (4). Similarly, the expressions for $\mathcal{B}^{c}\left(T_{k, l}(\rho)\right), \mathcal{B}^{o}\left(T_{k, l}(\rho)\right)$, and $\mathcal{J}\left(T_{k, l}(\rho)\right)$ follow from (7). Since the truncation is also additive for good $\mathcal{Z}$-representations, part (b) follows from (6).

Krull-Remak-Schmidt decomposition for good $\mathcal{Z}$ - representations:

If $\rho$ has finite support this is the standard Krull-Remak-Schmidt decomposition theorem in an abelian category.

If $\rho$ is periodic it is isomorphic to some $\tilde{\rho}^{\prime}$ with $\rho^{\prime}$ a $G_{2 m}$-representation. neither $m$ nor $\rho^{\prime}$ is unique. Clearly a decomposition of $\rho^{\prime}$ as sum of the barcoderepresentations $I_{1}^{\prime}$ with multiplicity $r_{1}^{\prime}, I_{2}^{\prime}$ with multiplicity $r_{2}^{\prime} \ldots I_{N^{\prime}}^{\prime}$ with multiplicity $r_{N_{1}^{\prime}}^{\prime}$ and Jordan blocks whose total dimension of the underlying vector space $n_{1}^{\prime}$ provides a decomposition of $\rho$ as an infinite sum of $I_{1}^{\prime}+m k$ with multiplicity $r_{1}^{\prime}$, $I_{2}^{\prime}+m k$ with multiplicity $r_{2}^{\prime} \ldots I_{N^{\prime}}^{\prime}+m k$ with multiplicity $r_{N_{1}^{\prime}}^{\prime}$ for any $k \in \mathbb{Z}$ and

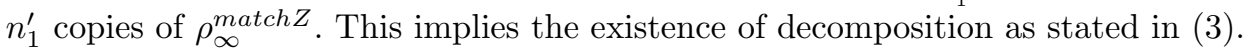

Note that for any decomposition of type (3) the following holds:

- there are only finitely many barcodes up to translation by multiples of $m$ which makes the length of bar codes bounded from above,

- each barcode appears with finite multiplicity and

- there are finitely many components $\rho_{\infty}^{\mathcal{Z}}$.

Since a truncation $T_{i, j}$ converts a barcode into a barcode (possibly empty) and $\rho^{\mathcal{Z}_{\infty}}$ into a closed barcode $[i, j]$, comparing the outcome of enough many truncation $T_{i, j}$ (with $(j-i)$ larger than the length of the barcodes of the two representations) and in view of the validity of the Krull-Remak-Schmidt theorem for finite graphs, on obtains the equality in the number of each type of barcodes and of the number of components $\rho_{\infty}^{\mathcal{Z}}$ in any two decompositions $[3]$.

2.3. The matrix $M(\rho)$ and the representation $\rho_{u}$. For every $G_{2 m}$-representation $\rho=\left\{V_{r}, \alpha_{i}, \beta_{i}\right\}, 1 \leq r \leq 2 m, 1 \leq i \leq m$, we introduce a linear map, $M(\rho): \bigoplus_{1 \leq i \leq m} V_{2 i-1} \rightarrow$ $\bigoplus_{1 \leq i \leq m} V_{2 i}$, defined by the block matrix:

$$
\left(\begin{array}{ccccc}
\alpha_{1} & -\beta_{1} & 0 & \ldots & 0 \\
0 & \alpha_{2} & -\beta_{2} & \ddots & \vdots \\
\vdots & \ddots & \ddots & \ddots & 0 \\
0 & \ldots & 0 & \alpha_{m-1} & -\beta_{m-1} \\
-\beta_{m} & 0 & \ldots & 0 & \alpha_{m}
\end{array}\right) .
$$

Moreover, for $u \in \kappa \backslash 0$ we let $\rho_{u}=\left\{V_{r}^{\prime}, \alpha_{i}^{\prime}, \beta_{i}^{\prime}\right\}$ denote the $G_{2 m}$-representation where $V_{r}^{\prime}=V_{r}, \alpha_{1}^{\prime}=u \alpha_{1}, \alpha_{i}^{\prime}=\alpha_{i}$ for $i \neq 1$ and $\beta_{i}^{\prime}=\beta_{i}$.

For a $\mathcal{Z}$-representation $\rho=\left\{V_{r}, \alpha_{i}, \beta_{i}\right\}$ the linear map $M(\rho): \bigoplus_{i \in \mathbb{Z}} V_{2 i-1} \rightarrow$ $\bigoplus_{i \in \mathbb{Z}} V_{2 i}$, is defined by the infinite block matrix with entries:

$$
M(\rho)_{2 r-1,2 s}= \begin{cases}\alpha_{r} & \text { if } s=r, \\ \beta_{r-1} & \text { if } s=r-1, \text { and } \\ 0 & \text { otherwise. }\end{cases}
$$


If the $\mathcal{Z}$-representation $\tilde{\rho}$ is the infinite cyclic covering of a $G_{2 m}$-representation $\rho$, then the shift $i \mapsto i+m$ defines isomorphisms $t_{\text {odd }}: \bigoplus_{i \in \mathbb{Z}} V_{2 i-1} \rightarrow \bigoplus_{i \in \mathbb{Z}} V_{2(i+m)-1}$ and $t_{\text {even }}: \bigoplus_{i \in \mathbb{Z}} V_{2 i} \rightarrow \bigoplus_{i \in \mathbb{Z}} V_{2(i+m)}$ such that $t_{\text {even }} \circ M(\tilde{\rho})=M(\tilde{\rho}) \circ t_{\text {odd }}$. The induced automorphisms on $\operatorname{ker} M(\tilde{\rho})$ and coker $M(\tilde{\rho})$ will be denoted by:

$$
t: \operatorname{ker} M(\tilde{\rho}) \rightarrow \operatorname{ker} M(\tilde{\rho}) \quad \text { and } \quad t: \operatorname{coker} M(\tilde{\rho}) \rightarrow \operatorname{coker} M(\tilde{\rho}) .
$$

For every $\Gamma$-representation $\rho$ introduce an $\mathbb{N}_{0}$-valued function $\operatorname{dim} \rho$ on the set of vertices of $\Gamma$, defined by $\operatorname{dim} \rho(x):=\operatorname{dim} V_{x}$, where $V_{x}$ is the vector space assigned to the vertex $x$ by $\rho$. Moreover, for every representation $\rho$ of $G_{2 m}$ or $\mathcal{Z}$ we put $\operatorname{dim} \operatorname{ker}(\rho):=\operatorname{dim} \operatorname{ker} M(\rho)$ and $\operatorname{dim} \operatorname{coker}(\rho):=\operatorname{dim} \operatorname{coker} M(\rho)$.

As noticed in [3] one has:

Lemma $2.3\left([3)\right.$. Suppose $\rho, \rho_{1}$ and $\rho_{2}$ are representations of $G_{2 m}$ or $\mathcal{Z}$. Then the following hold true for $\lambda, u \in \kappa \backslash 0, k \in \mathbb{N}$, and all intervals $I$ with integral endpoints:

(a) $\operatorname{dim}\left(\rho_{u}\right)=\operatorname{dim}(\rho)$.

(b) $\left(\rho_{1} \oplus \rho_{2}\right)_{u}=\left(\rho_{1}\right)_{u} \oplus\left(\rho_{2}\right)_{u}$.

(c) $\rho^{G}(\lambda, k)_{u}=\rho^{G}(u \lambda, k)$.

(d) $\rho^{G}(I)_{u}=\rho^{G}(I)$.

(e) $\operatorname{dim}\left(\rho_{1} \oplus \rho_{2}\right)=\operatorname{dim}\left(\rho_{1}\right)+\operatorname{dim}\left(\rho_{2}\right)$.

(f) $\operatorname{dim} \operatorname{ker}\left(\rho_{1} \oplus \rho_{2}\right)=\operatorname{dim} \operatorname{ker}\left(\rho_{1}\right)+\operatorname{dim} \operatorname{ker}\left(\rho_{2}\right)$.

(g) $\operatorname{dim} \operatorname{coker}\left(\rho_{1} \oplus \rho_{2}\right)=\operatorname{dim} \operatorname{coker}\left(\rho_{1}\right)+\operatorname{dim} \operatorname{coker}\left(\rho_{2}\right)$.

Proof. The statements in parts (a) and (b) are trivial. Part (c) follows from the fact that the Jordan block $T(u \lambda, k)$, see (1), is conjugate to $u T(\lambda, k)$ via the diagonal matrix $\operatorname{diag}\left(1, u, u^{2}, \ldots, u^{k-1}\right)$. Part (d) readily follows from the classification of indecomposable $G_{2 m}$-representations. The statement in (e) is obvious. To see (f) and $(\mathrm{g})$ note that $M\left(\rho_{1} \oplus \rho_{2}\right)=M\left(\rho_{1}\right) \oplus M\left(\rho_{2}\right)$, hence $\operatorname{ker}\left(\rho_{1} \oplus \rho_{2}\right)=\operatorname{ker}\left(\rho_{1}\right) \oplus$ $\operatorname{ker}\left(\rho_{2}\right)$ and $\operatorname{coker}\left(\rho_{1} \oplus \rho_{2}\right)=\operatorname{coker}\left(\rho_{1}\right) \oplus \operatorname{coker}\left(\rho_{2}\right)$. For $G_{2 m}$-representations the latter can be found in [3. Proposition 4.1].

Moreover:

Proposition $2.4([3])$.

(a) For indecomposable $G_{2 m}$-representations of type I we have

(a1) $\operatorname{dim} \operatorname{ker} \rho^{G}([i, j])=0$, dim coker $\rho^{G}([i, j])=1$,

(a2) $\operatorname{dim} \operatorname{ker} \rho^{G}([i, j))=0$, dim coker $\rho^{I}([i, j))=0$,

(a3) $\operatorname{dim} \operatorname{ker} \rho^{G}((i, j])=0, \operatorname{dim} \operatorname{coker} \rho^{G}((i, j])=0$,

(a4) $\operatorname{dim} \operatorname{ker} \rho^{G}((i, j))=1, \operatorname{dim} \operatorname{coker} \rho^{G}((i, j))=0$,

and for indecomposable $\mathcal{Z}$-representations with finite support:

(a5) $\operatorname{dim} \operatorname{ker} \rho^{\mathcal{Z}}([i, j])=0$, dim coker $\rho^{\mathcal{Z}}([i, j])=1$,

(a6) $\operatorname{dim} \operatorname{ker} \rho^{\mathcal{Z}}([i, j))=0$, dim coker $\rho^{\mathcal{Z}}([i, j))=0$,

(ał) $\operatorname{dim} \operatorname{ker} \rho^{\mathcal{Z}}((i, j])=0$, dim coker $\rho^{\mathcal{Z}}((i, j])=0$,

(a8) $\operatorname{dim} \operatorname{ker} \rho^{\mathcal{Z}}((i, j))=1, \operatorname{dim} \operatorname{coker} \rho^{\mathcal{Z}}((i, j))=0$.

(b) For indecomposable $G_{2 m}$-representations of type II we have

(b1) $\operatorname{dim} \operatorname{ker} \rho^{G}(J)=0$ if $J \neq\left(\kappa^{k}, T(1, k)\right)$; dim $\operatorname{ker} \rho^{G}\left(\kappa^{k}, T(1, k)\right)=1$

(b2) $\operatorname{dim} \operatorname{coker} \rho^{G}(J)=0$ if $J \neq\left(\kappa^{k}, T(1, k)\right)$; dim coker $\rho^{G}\left(\kappa^{k}, T(1, k)\right)=1$

and for the $\mathcal{Z}$-representation $\rho_{\infty}^{\mathcal{Z}}$ :

(b3) $\operatorname{dim} \operatorname{ker}\left(\rho_{\infty}^{\mathcal{Z}}\right)=0$,

(b4) $\operatorname{dim} \operatorname{coker}\left(\rho_{\infty}^{\mathcal{Z}}\right)=1$. 
Proof. The statements in (a1), (a2), (a3), (a4), (b1), and (b2) can be found in 3. Proposition 4.3]. Parts (a5), (a6), (a7), (a8), (b3), and (b4) can be proved analogously. Indeed, the calculation of the kernel of $M(\rho)$ reduces to the description of the space of solutions of the linear system:

$$
\begin{aligned}
\alpha_{1}\left(v_{1}\right) & =\beta_{1}\left(v_{3}\right) \\
\alpha_{2}\left(v_{3}\right) & =\beta_{2}\left(v_{5}\right) \\
& \vdots \\
\alpha_{m}\left(v_{2 m-1}\right) & =\beta_{m}\left(v_{1}\right)
\end{aligned}
$$

wit $v_{2 i-1} \in V_{2 i-1}$. This is straight forward for indecomposable representations.

Lemma 2.5. If $\rho=\left\{V_{i}, \alpha_{i}, \beta_{i}\right\}$ is a regular $\mathcal{Z}$-representation, i.e. all $\alpha_{i}$ and $\beta_{i}$ are isomorphisms, then ker $M(\rho)=0$, and for every $i$ the canonical inclusion $V_{2 i} \rightarrow$ $\bigoplus_{r \in \mathbb{Z}} V_{2 r}$ followed by the projection onto coker $M(\rho)$ provides an isomorphism $V_{2 i} \cong$ coker $M(\rho)$.

Proof. By regularity, the system of equations $\alpha_{r}\left(v_{2 r-1}\right)=\beta_{r}\left(v_{2 r+1}\right)$ does not have a non-trivial solution for which only finitely many of the $v_{2 r-1} \in V_{2 r-1}$ are nontrivial, whence ker $M(\rho)=0$. To see that $V_{2 i}$ intersects the image of $M(\rho)$ trivially, suppose $w \in V_{2 i} \cap \operatorname{img} M(\rho)$. Then there exist $v_{2 r-1} \in V_{2 r-1}$, almost all zero, such that $w=\alpha_{i}\left(v_{2 i-1}\right)-\beta_{i}\left(v_{2 i+1}\right)$ and $\alpha_{r}\left(v_{2 r-1}\right)=\beta_{r}\left(v_{2 r+1}\right)$ for all $r \neq i$. As $v_{2 r+1}=0$ for sufficiently large $r$, the latter equations imply $v_{2 i+1}=0$. Similarly, since $v_{2 r-1}=0$ for sufficiently small $r$, we obtain $v_{2 i-1}=0$, whence $w=0$. Finally, if $u \in V_{2 r}$, then the corresponding element in coker $M(\rho)$ can also be represented by by an element in $V_{2 r+2}$, namely $\alpha_{r+1} \beta_{r}^{-1}(u)$. Consequently, each element in coker $M(\rho)$ can be represented by an element in $V_{2 i}$.

To formulate a refinement of Proposition 2.4 we introduce additional notation:

Definition 2.6. For a set $S$ denote by $\kappa[S]$ the vector space generated by $S$, i.e. the vector space of $\kappa$-valued maps on $S$ with finite support, and by $\kappa[[S]]$ the vector space of all $\kappa$-valued maps on $S$. If $S$ is finite, then $\kappa[S]=\kappa[[S]]$.

For two subsets $S_{1}$ and $S_{2}$ of $S$ the canonical linear maps $\kappa\left[S_{1}\right] \rightarrow \kappa\left[S_{2}\right], \kappa\left[S_{1}\right] \rightarrow$ $\kappa\left[\left[S_{2}\right]\right]$, or $\kappa\left[\left[S_{1}\right]\right] \rightarrow \kappa\left[\left[S_{2}\right]\right]$ are the unique linear maps which restrict to the identity on $S_{1} \cap S_{2}$ and to zero on $S_{1} \backslash S_{2}$.

We warn the reader of the "unfortunate notational similarity" between $\kappa[S]$ and $\kappa\left[T^{-1}, T\right]$ with the last one denoting the ring of Laurent polynomials of variable $T$. Fortunately they appear below in contexts which exclude confusion.

If $\rho=\left\{V_{r}, \alpha_{i}, \beta_{i}\right\}$ is a $G_{2 m}$-representation, then the diagram

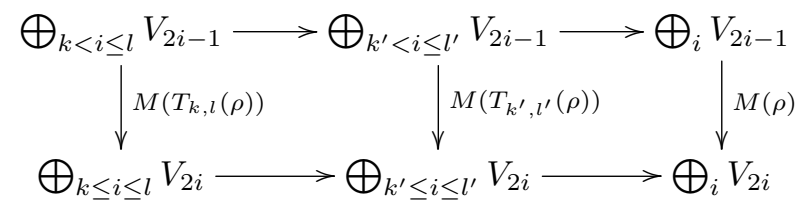

commutes, for all integers $1 \leq k^{\prime} \leq k \leq l \leq l^{\prime} \leq m$, and we obtain induced linear maps

$$
\operatorname{ker} M\left(T_{k, l}(\rho)\right) \stackrel{i}{\longrightarrow} \operatorname{ker} M\left(T_{k^{\prime}, l^{\prime}}(\rho)\right) \stackrel{i^{\prime}}{\longrightarrow} \operatorname{ker} M(\rho)
$$


as well as

$$
\operatorname{coker} M\left(T_{k, l}(\rho)\right) \stackrel{j}{\longrightarrow} \operatorname{coker} M\left(T_{k^{\prime}, l^{\prime}}(\rho)\right) \stackrel{j^{\prime}}{\longrightarrow} \operatorname{coker} M(\rho) .
$$

The same holds true if $\rho$ is a good $\mathcal{Z}$-representation and $k^{\prime} \leq k \leq l \leq l^{\prime}$. For either representation, the linear maps $i$ and $i^{\prime}$ in 10 are injective since the horizontal inclusions in diagram (9) are injective. The maps $j$ and $j^{\prime}$ in (11) need not be injective in general. Correspondingly, see Definition 2.6. we consider the linear maps

$$
\kappa\left[\mathcal{B}^{o}\left(T_{k, l}(\rho)\right)\right] \longrightarrow \kappa\left[\mathcal{B}^{o}\left(T_{k^{\prime}, l^{\prime}}(\rho)\right)\right] \longrightarrow \kappa\left[\mathcal{B}^{\circ}(\rho) \sqcup \mathcal{J}\right]
$$

and

$$
\kappa\left[\mathcal{B}^{c}\left(T_{k, l}(\rho)\right)\right] \longrightarrow \kappa\left[\mathcal{B}^{c}\left(T_{k^{\prime}, l^{\prime}}(\rho)\right)\right] \longrightarrow \kappa\left[\mathcal{B}^{c}(\rho) \sqcup \mathcal{J}\right]
$$

where $\mathcal{J}$ is defined as follows. In $(12) \mathcal{J}=\emptyset$ if $\rho$ is a good $\mathcal{Z}$-representation and $\mathcal{J}=\mathcal{J}_{1}(\rho)$ if $\rho$ is a $G_{2 m}$-representation. In 13$) \mathcal{J}=\mathcal{J}(\rho)$ if $\rho$ is a $\operatorname{good} \mathcal{Z}$ representation and $\mathcal{J}=\mathcal{J}_{1}(\rho)$ if $\rho$ is a $G_{2 m}$-representation. The linear maps in (12) are injective since, according to Lemma 2.2, we have inclusions $\mathcal{B}^{\circ}\left(T_{k, l}(\rho)\right) \subseteq$ $\mathcal{B}^{\circ}\left(T_{k^{\prime}, l^{\prime}}(\rho)\right) \subseteq \mathcal{B}^{o}(\rho) \subseteq \mathcal{B}^{\circ}(\rho) \sqcup \mathcal{J}$. Lemma 2.2 also shows that the linear maps in (13) will not be injective in general. Using decompositions as in (2) and (3), additivity of the constructions, and Proposition 2.4. we see that there are linear isomorphisms

$$
\begin{array}{ll}
\kappa\left[\mathcal{B}^{o}\left(T_{k, l}(\rho)\right)\right] \cong \operatorname{ker} M\left(T_{k, l}(\rho)\right), \quad \kappa\left[\mathcal{B}^{o}(\rho) \sqcup \mathcal{J}\right] \cong \operatorname{ker} M(\rho), \\
\kappa\left[\mathcal{B}^{c}\left(T_{k, l}(\rho)\right)\right] \cong \operatorname{coker} M\left(T_{k, l}(\rho)\right), \quad \kappa\left[\mathcal{B}^{c}(\rho) \sqcup \mathcal{J}\right] \cong \operatorname{coker} M(\rho),
\end{array}
$$

and that the ranks of the linear maps in 10 and 11 coincide with the ranks of the corresponding linear maps in (12) and (13), respectively. The following refinement of Proposition 2.4 asserts that these isomorphisms may even be chosen to be compatible with truncation.

Proposition 2.7. (a) Let $\rho$ be a $G_{2 m}$-representation. Then every decomposition $\rho=\bigoplus_{I \in \mathcal{B}(\rho)} \rho^{G}(I) \oplus \bigoplus_{J \in \mathcal{J}(\rho)} \rho^{G}(J)$ induces isomorphisms $\Psi^{o}, \Psi_{k, l}^{o}, \Psi^{c}$, and $\Psi_{k, l}^{c}$ such that the diagrams

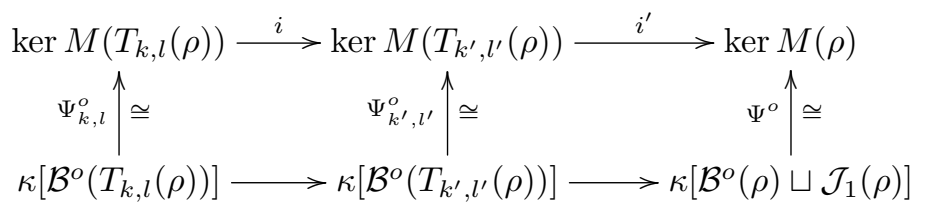

and

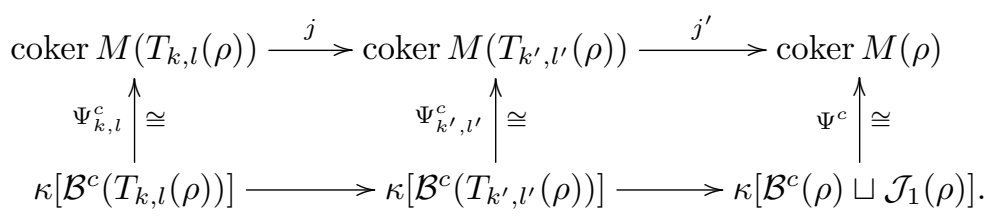

commute for all integers $1 \leq k^{\prime} \leq k \leq l \leq l^{\prime} \leq m$.

(b) Let $\rho$ be a good $\mathcal{Z}$-representation. Then every decomposition $\rho=\bigoplus_{I \in \mathcal{B}(\rho)} \rho(I) \oplus$ $\bigoplus_{n} \rho_{\infty}^{\mathcal{Z}}$, where $n=\sharp J(\rho)$, induces isomorphisms $\Psi^{o}, \Psi_{k, l}^{o}, \Psi^{c}$, and $\Psi_{k, l}^{c}$ such that 
the diagrams

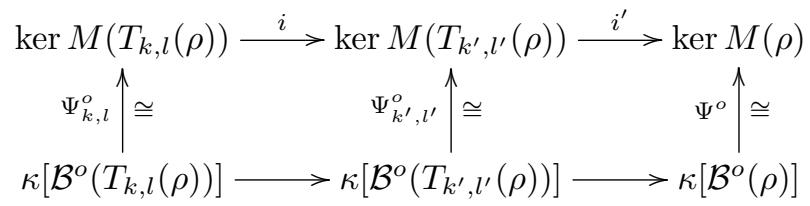

and

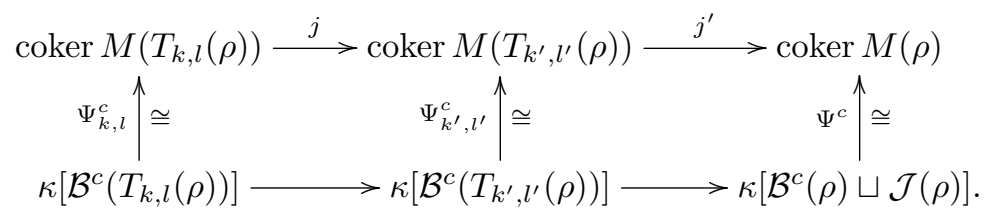

commute for all integers $k^{\prime} \leq k \leq l \leq l^{\prime}$.

Proof. Since the involved constructions are all additive, that is to say, compatible with direct sums of representations, it suffices to construct the isomorphisms $\Psi^{o}, \Psi_{k, l}^{o}, \Psi^{c}$, and $\Psi_{k, l}^{c}$ for indecomposable representations. For indecomposable representations, however, the constructions are tautological in view of Proposition 2.4 .

We close this section with an observation about the infinite cyclic covering associated with a $G_{2 m}$-representation. Let $\rho=\left\{V_{r}, \alpha_{i}, \beta_{i}\right\}$ be a $G_{2 m}$-representation and let $\tilde{\rho}=\left\{\tilde{V}_{r}, \tilde{\alpha}_{i}, \tilde{\beta}_{i}\right\}$ denote the associated infinite cyclic covering $\mathcal{Z}$-representation, cf. the beginning of Section 2.2. Recall that the shift by $m$ induces automorphisms denoted by $t$ on $\operatorname{ker} M(\tilde{\rho})$ and coker $M(\tilde{\rho})$, see (8). These automorphisms turn ker $M(\tilde{\rho})$ and coker $M(\tilde{\rho})$ into $\kappa\left[T^{-1}, T\right]$-modules such that $T$ acts by $t$ and $T^{-1}$ acts by $t^{-1}$. Appendix $\mathrm{C}$ contains some basic facts on $\kappa\left[T, T^{-1}\right]$-modules.

Correspondingly, the translation of intervals, $I \mapsto I+m$, induces bijections on $\mathcal{B}^{o}(\tilde{\rho})$ and $\mathcal{B}^{c}(\tilde{\rho})$, see $(4)$. The induced automorphisms on $\kappa\left[\mathcal{B}^{o}(\tilde{\rho})\right]$ and $\kappa\left[\mathcal{B}^{c}(\tilde{\rho})\right]$ turn these two vector spaces into $\kappa\left[T^{-1}, T\right]$-modules. Moreover, identifying $\kappa[\mathcal{J}(\tilde{\rho})]=$ $\bigoplus_{(V, T) \in \mathcal{J}(\rho)} V$, we obtain an automorphism $\bigoplus_{(V, T) \in \mathcal{J}(\rho)} T$ on $\kappa[\mathcal{J}(\tilde{\rho})]$ which we use to turn this vector space into a $\kappa\left[T^{-1}, T\right]$-module. Via $\kappa\left[\mathcal{B}^{c}(\tilde{\rho}) \sqcup \mathcal{J}(\tilde{\rho})\right]=$ $\kappa\left[\mathcal{B}^{c}(\tilde{\rho})\right] \oplus \kappa[\mathcal{J}(\tilde{\rho})]$, we obtain a $\kappa\left[T^{-1}, T\right]$-module structure on $\kappa\left[\mathcal{B}^{c}(\tilde{\rho}) \sqcup \mathcal{J}(\tilde{\rho})\right]$.

Lemma 2.8. Let $\rho$ be a $G_{2 m}$-representation and let $\tilde{\rho}$ denote the associated infinite cyclic covering $\mathcal{Z}$-representation. Then the following hold true:

a) The linear isomorphisms

$$
\Psi^{o}: \kappa\left[\mathcal{B}^{o}(\tilde{\rho})\right] \rightarrow \operatorname{ker} M(\tilde{\rho}) \quad \text { and } \quad \Psi^{c}: \kappa\left[\mathcal{B}^{c}(\tilde{\rho}) \sqcup \mathcal{J}(\tilde{\rho})\right] \rightarrow \operatorname{coker} M(\tilde{\rho})
$$

in Proposition 2.7(b) are isomorphisms of $\kappa\left[T^{-1}, T\right]$-modules (since by construction compatible with the $m$-periodicity).

b) The modules $\kappa\left[\mathcal{B}^{o}(\tilde{\rho})\right]$ and $\kappa\left[\mathcal{B}^{c}(\tilde{\rho})\right]$ are free. More precisely, we have isomorphisms of $\kappa\left[T^{-1}, T\right]$-modules

$$
\kappa\left[\mathcal{B}^{o}(\tilde{\rho})\right] \cong \kappa\left[T^{-1}, T\right]\left[\mathcal{B}^{o}(\rho)\right] \quad \text { and } \quad \kappa\left[\mathcal{B}^{c}(\tilde{\rho})\right] \cong \kappa\left[T^{-1}, T\right]\left[\mathcal{B}^{c}(\rho)\right] .
$$

c) The torsion part of the $\kappa\left[T^{-1}, T\right]$-module coker $M(\tilde{\rho})$ equipped with the automorphism induced by $T$ is isomorphic to the monodromy of the representation $\rho$, that is, $\kappa[\mathcal{J}(\tilde{\rho})] \cong \bigoplus_{J \in \mathcal{J}(\rho)} J$. 
Proof. Since the statement is additive in the $G_{2 m}$-representation $\rho$, it suffices to consider indecomposable $G_{2 m}$-representations $\rho$. Part c) follows from Lemma 2.5 Indeed if $\rho$ is a barcode representation the result follows from Proposition 2.4 In this case both $\operatorname{ker}(M(\tilde{\rho}))$ and $\operatorname{coker}(M(\tilde{\rho}))$ are free of rank 1 or 0 . and there is no regular part of $\rho$. If $\rho$ is a Jordan block, hence $\rho$ is regular, the result follows from Lemma 2.5

\section{BAR CODES AND JORDAN BLOCKS VIA GRAPH REPRESENTATIONS}

In this section we will describe graph representations associated with a tame circle valued map. Furthermore, we will establish fundamental exact sequences that permit to compute the (twisted) homology of the underlying space in terms of the corresponding barcodes and Jordan blocks.

Let $f: X \rightarrow S^{1}$ be a tame map and $0<\theta_{1}<\theta_{2}<\cdots<\theta_{m} \leq 2 \pi$ be the critical angles (the angles of the set $\Sigma$ in the definition of tameness). Choose the regular values $t_{1}<t_{2}<\cdots<t_{m}$ with $\theta_{i-1}<t_{i}<\theta_{i}$ and $0<t_{1}<\theta_{1}$. In order to differentiate between regular and singular fibers we write $R_{i}:=f^{-1}\left(t_{i}\right)$ and $X_{i}:=f^{-1}\left(\theta_{i}\right)$.

The tameness of $f$ induces the maps $a_{i}: R_{i} \rightarrow X_{i}$ for $1 \leq i \leq m, b_{i}: R_{i+1} \rightarrow X_{i}$ for $i \leq m-1$ and $b_{m}: R_{1} \rightarrow X_{m}$ which are unique up to homotopy; this means that different choices of the regular values, say $t_{i}^{\prime}$ instead of $t_{i}$, lead to homotopy equivalences $\omega_{i}: R_{i} \rightarrow R_{i}^{\prime}$ s.t. $a_{i}^{\prime} \cdot \omega_{i}$ is homotopic to $a_{i}$ and $b_{i}^{\prime} \cdot \omega_{i}$ is homotopic to $b_{i}$.

Indeed the fiber $R_{i}$ identifies up to homotopy to regular fibers $f^{-1}(t)$ and $f^{-1}\left(t^{\prime}\right)$, $\theta_{i-1}<t<t^{\prime}<\theta_{i}$ since $f^{-1}\left(\theta_{i-1}, \theta_{i}\right) \rightarrow\left(\theta_{i-1}, \theta_{i}\right)$ is a fibration. One chooses $t$ and $t^{\prime}$ to make sure that $f^{-1}(t)$ and $f^{-1}\left(t^{\prime}\right)$ are contained in open sets which retract to $X_{i}$ resp. $X_{i-1}$. The maps $b_{i-1}$ and $a_{i}$ are the composition of such identifications with the retractions to $X_{i-1}$ resp. $X_{i}$. We leave the reader to do the tedious verification that the homotopy classes of $a_{i}$ and $b_{i-1}$ are independent of the choices made.

Passing to $r$-homology one obtains the $G_{2 m}$-representation $\rho_{r}=\rho_{r}(f)$ whose vector spaces are $V_{2 s}=H_{r}\left(X_{s}\right)$ and $V_{2 s-1}=H_{r}\left(R_{s}\right)$ and the linear maps $\alpha_{i}$ and $\beta_{i}$ are induced by the continuous maps $a_{i}$ and $b_{i}$.

The representation $\rho_{r}(f)$ has bar codes whose ends are $i, j+k m, 1 \leq i, j \leq m$. Denote by $\mathcal{B}_{r}(f)$, the collections of intervals defined by the bar codes of $\rho_{r}(f)$ but with the ends $i$ and $j+k m$ replaced by $\theta_{i}$ and $\theta_{j}+2 \pi k$. Denote by $\mathcal{J}_{r}(f)$ the collection of Jordan blocks of the representation $\rho_{r}(f)$.

If $\tilde{f}: \tilde{X} \rightarrow \mathbb{R}$ is the infinite cyclic covering of $f$ then the real numbers $\theta_{i}+2 \pi k$ are the critical values and $t_{i}+2 \pi k$ are regular values (between consecutive critical values) and the tameness of $\tilde{f}$ gives the maps $a_{i+k m}: \tilde{X}_{t_{i+1}+2 \pi k} \rightarrow \tilde{X}_{\theta_{i+2 \pi k}}$ and $b_{i+k m}: \tilde{X}_{t_{i}+2 \pi k} \rightarrow \tilde{X}_{\theta_{i}+2 \pi k}$. By passing to homology in dimension $r$ one obtains a good $\mathcal{Z}$-representation $\rho_{r}(\tilde{f})$ which is exactly the infinite cyclic covering $\widetilde{\rho_{r}(f)}$.

The collections $\mathcal{B}_{r}(\tilde{f}), \mathcal{B}_{r}^{c}(\tilde{f}), \mathcal{B}_{r}^{o}(\tilde{f}), \mathcal{B}_{r}^{c o}(\tilde{f}), \mathcal{B}_{r}^{o c}(\tilde{f})$ also denoted by $\tilde{\mathcal{B}}_{r}(f), \tilde{\mathcal{B}}_{r}^{c}(f)$, $\tilde{\mathcal{B}}_{r}^{o}(f), \tilde{\mathcal{B}}_{r}^{c o}(f), \tilde{\mathcal{B}}_{r}^{o c}(f)$ are the bar codes of the representation $\widetilde{\rho_{r}(f)}$. They are invariant w.r. to the $2 \pi$ translation and the collections $\mathcal{B}_{r}(f), \mathcal{B}_{r}^{c}(f), \mathcal{B}_{r}^{o}(f), \mathcal{B}_{r}^{c o}(f)$, $\mathcal{B}_{r}^{o c}(f)$ can be viewed as equivalence classes (modulo the $2 \pi$ translation) of elements of $\mathcal{B}_{r}^{c}(\tilde{f}), \mathcal{B}_{r}^{o}(\tilde{f}), \mathcal{B}_{r}^{c o}(\tilde{f}), \mathcal{B}_{r}^{o c}(\tilde{f})$. For $X$ compact and $f$ tame the sets $\mathcal{B}_{r}(f)$ are finite while $\tilde{\mathcal{B}}_{r}(f)$, if nonempty, are infinite. 
Given $\xi \in H^{1}(X ; \mathbb{Z})$ and $u \in \kappa \backslash 0$, the pair $(\xi, u)$ denotes the rank one representation $H_{1}(X ; \mathbb{Z}) \rightarrow \mathbb{Z} \rightarrow \kappa \backslash 0$, where the first arrow is given by $\xi$ and the second by the homomorphism $\langle u\rangle: \mathbb{Z} \rightarrow \kappa \backslash 0$ defined by $\langle u\rangle(n)=u^{n}$. One denotes by $H_{r}(X ;(\xi, u))$ the homology of $X$ with coefficients in the local system defined by the representation $(\xi, u)$, see [21, Section 3.H]. To describe the latter homology group, recall that the singular chain complex of the infinite cyclic covering, $C_{*}(\tilde{X})$, can be regarded as a chain complex of $\kappa\left[T^{-1}, T\right]$-modules where the action of $T$ is induced by the fundamental deck transformation. The homology $H_{r}(X ;(\xi, u))$ is canonically isomorphic to the $r$-th homology of the $\kappa$-cochain complex $C_{*}(\tilde{X}) \otimes_{u} \kappa$ obtained by tensorizing with the representation $\kappa\left[T^{-1}, T\right] \rightarrow \kappa$ determined by $T \mapsto u$. If $u=1$, then we have a canonical isomorphism $C_{*}(\tilde{X}) \otimes_{u} \kappa=C_{*}(X)$ and thus $H_{r}(X ;(\xi, 1))=H_{r}(X)$.

Replacing homology by homology with coefficients in the local system $(\xi, u)$ leads also to the replacement of the representations $\rho_{f}(f)$ with the representations $\rho_{r}(f)_{u}$. as explained below. Since the local system becomes trivial over $R_{i}$ and $X_{i}$, we have isomorphisms $H_{r}\left(R_{i} ;(\xi, u)\right) \cong H_{r}\left(R_{i}\right)$ and $H_{r}\left(X_{i} ;(\xi, u)\right) \cong H_{r}\left(X_{i}\right)$. The maps induced by $a_{i}$ and $b_{i}$, however, will not all coincide with the maps in the representation $\rho_{r}(f)$ but with the ones for $\rho_{r}(f)_{u}$. More precisely, every trivialization of the infinite cyclic covering over $\left[\theta_{1}, t_{1}+2 \pi\right]$, induces isomorphisms $\phi_{i}$ and $\phi_{i}^{\prime}, 1 \leq i \leq m$, such that the diagram

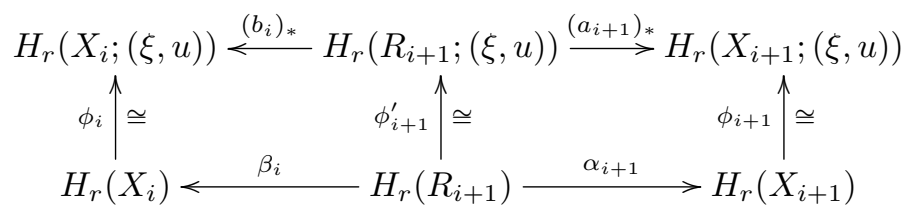

commutes for all $1 \leq i<m$, and the diagram

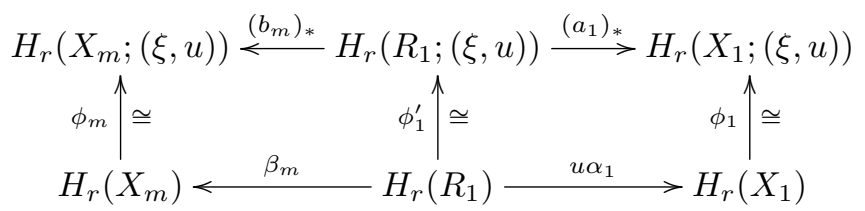

commutes. The $G_{2 m}$-representation obtained by using homology with coefficients in $(\xi, u)$ will thus be isomorphic to $\left(\rho_{r}(f)\right)_{u}$, see Section 2.3 .

3.1. The relevant exact sequences, cf. 3. The tool which permits the calculation of the homology of $X, \tilde{X}$ and various pieces of these spaces is provided by Proposition 3.1 below. The sequence in (19) has been established in [3, Section 5].

Proposition 3.1. Let $f: X \rightarrow \mathbb{S}^{1}$ be a tame map and $\tilde{f}: \tilde{X} \rightarrow \mathbb{R}$ its infinite cyclic covering. Let $\rho_{r}=\rho_{r}(f)$ and $\tilde{\rho}_{r}=\rho_{r}(\tilde{f})=\widetilde{\rho}_{r}(f)$ be the representations associated with $f$ and $\tilde{f}$. One has the following short exact sequences

$$
0 \rightarrow \operatorname{coker} M\left(\left(\rho_{r}\right)_{u}\right) \rightarrow H_{r}\left(X ;\left(\xi_{f}, u\right)\right) \rightarrow \operatorname{ker} M\left(\left(\rho_{r-1}\right)_{u}\right) \rightarrow 0,
$$

which for $u=1$ becomes

$$
0 \rightarrow \operatorname{coker} M\left(\rho_{r}\right) \rightarrow H_{r}(X) \rightarrow \operatorname{ker} M\left(\rho_{r-1}\right) \rightarrow 0 .
$$

Moreover, one has a short exact sequence of $\kappa\left[T^{-1}, T\right]$-modules

$$
0 \rightarrow \operatorname{coker} M\left(\tilde{\rho}_{r}\right) \rightarrow H_{r}(\tilde{X}) \rightarrow \operatorname{ker} M\left(\tilde{\rho}_{r-1}\right) \rightarrow 0 \text {. }
$$


These sequences are all compatible with truncations as explained below, see Diagrams 21) and 22.

Recall that the $\kappa\left[T^{-1}, T\right]$-module structure on $H_{r}(\tilde{X})$ is induced by the fundamental deck transformation. The $\kappa\left[T^{-1}, T\right]$-module structures on $\operatorname{ker}\left(\tilde{\rho}_{r}\right)$ and $\operatorname{coker}\left(\tilde{\rho}_{r}\right)$ have been described at the end of Section 2 .

Observe that for $\theta_{i} \leq \theta_{j}$ critical angles of $f$, if $f_{\left[\theta_{i}, \theta_{j}\right]}$ denotes the restriction of $f$ to $X_{\left[\theta_{i}, \theta_{j}\right]}=f^{-1}\left[\theta_{i}, \theta_{j}\right]$, then

$$
\rho_{r}\left(f_{\left[\theta_{i}, \theta_{j}\right]}\right)=T_{i, j}\left(\rho_{r}(f)\right) .
$$

Similarly, for $c_{i} \leq c_{j}$ critical values of $\tilde{f}$, if $\tilde{f}_{\left[c_{i}, c_{j}\right]}$ denotes the restriction of $\tilde{f}$ to $\tilde{X}_{\left[c_{i}, c_{j}\right]}=\tilde{f}^{-1}\left[c_{i}, c_{j}\right]$, then

$$
\rho_{r}\left(\tilde{f}_{\left[c_{i}, c_{j}\right]}\right)=T_{i, j}\left(\tilde{\rho}_{r}(f)\right) .
$$

Since $f$ and therefore $\tilde{f}$ is tame one also has:

for any $\theta^{\prime}$ with $\theta_{i-1}<\theta^{\prime} \leq \theta_{i}$ and $\theta^{\prime \prime}$ with $\theta_{j} \leq \theta^{\prime \prime}<\theta_{j+1}$

$$
\rho_{r}\left(f_{\left[\theta^{\prime}, \theta^{\prime \prime}\right]}\right)=\rho_{r}\left(f_{\left[\theta_{i}, \theta_{j}\right]}\right)
$$

and for any $c^{\prime}$ with $c_{i-1}<c^{\prime} \leq c_{i}$ and $c^{\prime \prime}$ with $c_{j} \leq c^{\prime \prime}<c_{j+1}$

$$
\rho_{r}\left(f_{\left[c^{\prime}, c^{\prime \prime}\right]}\right)=\rho_{r}\left(f_{\left[c_{i}, c_{j}\right]}\right) .
$$

In the case of the $G_{2 m}$-representation $\rho_{r}(f)$ "compatibility with truncation" means that for any pairs of critical angles $\left(\theta_{i}, \theta_{j}\right)$ and $\left(\theta_{i^{\prime}}, \theta_{j^{\prime}}\right), 0<\theta_{i} \leq \theta_{i^{\prime}} \leq \theta_{j^{\prime}} \leq$ $\theta_{j} \leq 2 \pi$ the following diagram is commutative:

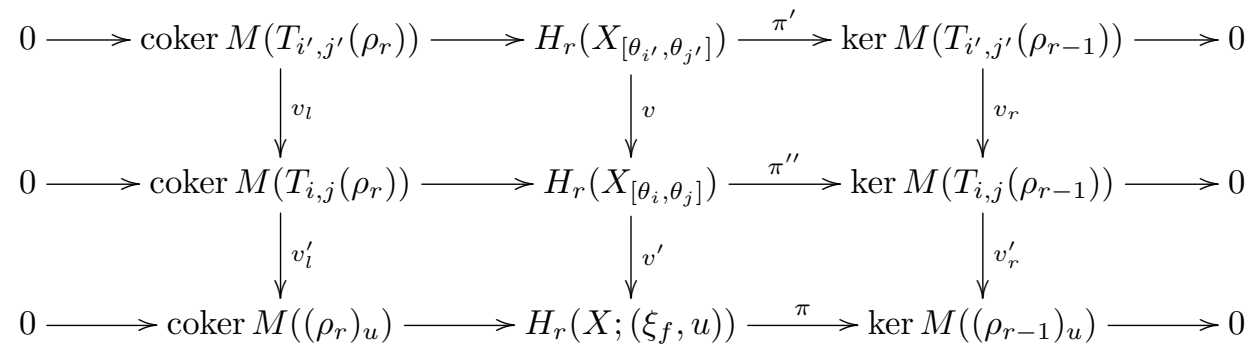

In the case of the $\mathcal{Z}$-representation $\tilde{\rho}_{r}$, this means that for any pairs of critical values $\left(c_{i}, c_{j}\right)$ and $\left(c_{i^{\prime}}, c_{j^{\prime}}\right)$ with $c_{i} \leq c_{i^{\prime}} \leq c_{j^{\prime}} \leq c_{j}$ the following diagram is commutative:

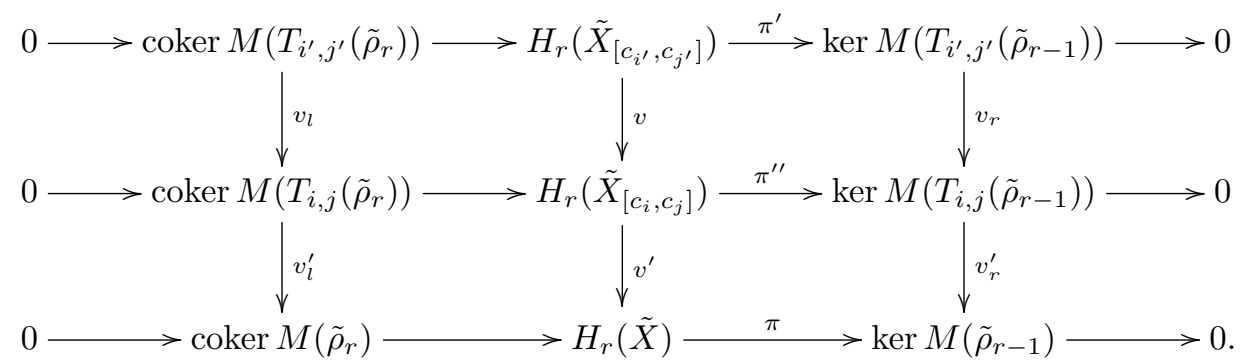

Note that diagrams 21 and 22 implies that any splitting (= right inverse) of $\pi^{\prime}$ or $\pi^{\prime \prime}$ extend to a splitting of $\pi^{\prime \prime}$ or $\pi$ respectively. 
Proof. The reader should recognize in the matrices $M\left(T_{i, j}\left(\tilde{\rho}_{r}\right)\right), M\left(\left(\rho_{r}\right)_{u}\right)$ and $M\left(\tilde{\rho}_{r}\right)$ the linear maps induced from $\bigoplus_{i \leq k \leq j} H_{r}\left(R_{k}\right)$ to $H_{r}\left(X_{\left[\theta_{i}, \theta_{j}\right]}\right)$, from $\bigoplus_{1 \leq i \leq m} H_{r}\left(R_{i}\right)$ to $H_{r}\left(X ;\left(\xi_{f}, u\right)\right)$ and from $\bigoplus_{1 \leq i \leq m} H_{r}\left(R_{i}\right)$ to $H_{r}(\tilde{X})$ respectively.

Denote by $\mathcal{R}:=\bigsqcup_{1 \leq i \leq m} R_{i}, \tilde{\mathcal{R}}:=\bigsqcup_{i \in \mathbb{Z}} R_{i}, \mathcal{X}:=\bigsqcup_{1 \leq i \leq m} X_{i}$ and $\tilde{\mathcal{X}}:=\bigsqcup_{i \in \mathbb{Z}} X_{i}$. The short exact sequence (18) follows from the long exact sequence

$$
\rightarrow H_{r}(\mathcal{R}) \stackrel{M\left(\left(\rho_{r}\right)_{u}\right)}{\longrightarrow} H_{r}(\mathcal{X}) \rightarrow H_{r}(X ;(\xi, u)) \rightarrow H_{r-1}(\mathcal{R}) \stackrel{M\left(\left(\rho_{r-1}\right)_{u}\right)}{\longrightarrow} H_{r-1}(\mathcal{X}) \rightarrow
$$

with $H_{r}(\mathcal{R})=\bigoplus_{1 \leq i \leq m} H_{r}\left(R_{i}\right)$ and $H_{r}(\mathcal{X})=\bigoplus_{1 \leq i \leq m} H_{r}\left(X_{i}\right)$, and the short exact sequence 201 follows from the long exact sequence

$$
\cdots \rightarrow H_{r}(\tilde{\mathcal{R}}) \stackrel{M\left(\rho_{r}\right)}{\longrightarrow} H_{r}(\tilde{\mathcal{X}}) \rightarrow H_{r}(\tilde{X}) \rightarrow H_{r-1}(\tilde{\mathcal{R}}) \stackrel{M\left(\rho_{r-1}\right)}{\longrightarrow} H_{r-1}(\tilde{\mathcal{X}}) \rightarrow \cdots
$$

which remain to be established. The sequence in 19 appears as a special case of the sequence in $(18)$ for $u=1$.

Since both long exact sequences (23) and 24) are derived in the same way we will treat only (23) and for simplicity only the case $u=1$.

First choose an $\epsilon>0$ small enough so that $2 \epsilon<t_{1}$ and $\theta_{i-1}+2 \epsilon<t_{i}<\theta_{i}-2 \epsilon$. To simplify the writing, since $i \leq m$, introduce $\theta_{m+1}=\theta_{1}+2 \pi$, let

$$
f^{-1}\left(\left[\theta_{m} \pm \epsilon, \theta_{m+1} \pm \epsilon\right)\right):=\tilde{f}^{-1}\left(\left[\theta_{m} \pm \epsilon, \theta_{1}+2 \pi \pm \epsilon\right]\right),
$$

and define

$$
\mathcal{P}^{\prime}:=\bigsqcup_{1 \leq i \leq m} f^{-1}\left(\left[\theta_{i}, \theta_{i+1}-\epsilon\right)\right), \quad \mathcal{P}^{\prime \prime}:=\bigsqcup_{1 \leq i \leq m} f^{-1}\left(\left(\theta_{i}+\epsilon, \theta_{i+1}\right]\right) .
$$

Observe that in view of the choice of $\epsilon$ and of the tameness of $f$ the inclusions $\mathcal{X} \subset \mathcal{P}^{\prime}, \mathcal{X} \subset \mathcal{P}^{\prime \prime}$, and $\mathcal{X} \sqcup \mathcal{R} \subset \mathcal{P}^{\prime} \cap \mathcal{P}^{\prime \prime}$ are homotopy equivalences. The MayerVietoris long exact sequence for $X=\mathcal{P}^{\prime} \cup \mathcal{P}^{\prime \prime}$ gives the commutative diagram

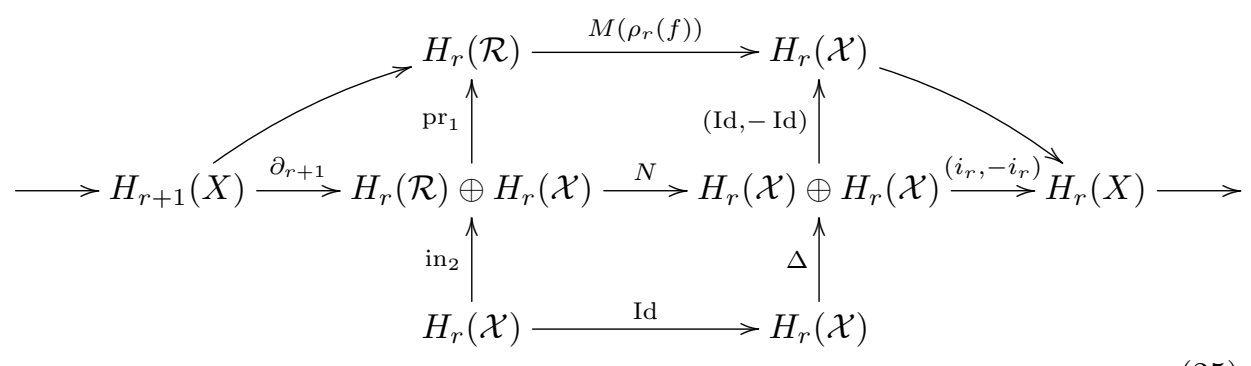

where $\Delta$ denotes the diagonal, $\mathrm{in}_{2}$ the inclusion on the second component, $\mathrm{pr}_{1}$ the projection on the first component, $i_{r}$ the linear map induced in homology by the inclusion $\mathcal{X} \subset X$. Recall that the matrix $M\left(\rho_{r}(f)\right)$ is defined by

$$
\left(\begin{array}{ccccc}
\alpha_{1}^{r} & -\beta_{1}^{r} & 0 & \cdots & 0 \\
0 & \alpha_{2}^{r} & -\beta_{2}^{r} & \ddots & \vdots \\
\vdots & \ddots & \ddots & \ddots & 0 \\
0 & \cdots & 0 & \alpha_{m-1}^{r} & -\beta_{m-1}^{r} \\
-\beta_{m}^{r} & 0 & \cdots & 0 & \alpha_{m}^{r}
\end{array}\right)
$$


with $\alpha_{i}^{r}: H_{r}\left(R_{i}\right) \rightarrow H_{r}\left(X_{i}\right)$ and $\beta_{i}^{r}: H_{r}\left(R_{i+1}\right) \rightarrow H_{r}\left(X_{i}\right)$ induced by the maps $a_{i}$ and $b_{i}$. The block matrix $N$ is defined by

$$
N:=\left(\begin{array}{cc}
\alpha^{r} & \mathrm{Id} \\
-\beta^{r} & \mathrm{Id}
\end{array}\right)
$$

where $\alpha^{r}$ and $\beta^{r}$ are the matrices

$$
\left(\begin{array}{cccc}
\alpha_{1}^{r} & 0 & \cdots & 0 \\
0 & \alpha_{2}^{r} & \ddots & \vdots \\
\vdots & \ddots & \ddots & 0 \\
0 & \cdots & 0 & \alpha_{m-1}^{r}
\end{array}\right) \text { and }\left(\begin{array}{ccccc}
0 & \beta_{1}^{r} & 0 & \ldots & 0 \\
0 & 0 & \beta_{2}^{r} & \ddots & \vdots \\
\vdots & \vdots & \ddots & \ddots & 0 \\
0 & 0 & \ldots & 0 & \beta_{m-1}^{r} \\
\beta_{m}^{r} & 0 & \ldots & 0 & 0
\end{array}\right)
$$

The long exact sequence 23 is the top sequence in the diagram (25).

The long exact sequence (24) can be established analogously. The naturality of the Mayer-Vietoris sequence w.r. to maps which preserve the decomposition of a space in two pieces implies that the homomorphisms in the sequence (24) intertwine the automorphisms induced by the fundamental deck transformation on $H_{r}(\tilde{\mathcal{R}}), H_{r}(\tilde{\mathcal{X}})$, and $H_{r}(\tilde{X})$, respectively. Hence, 20 is a short exact sequence of $\kappa\left[T^{-1}, T\right]$-modules. Compatibility with truncations follows from the naturality of the Mayer-Vietoris sequence too.

\section{Proof of Theorem 1.1 And some Refinements.}

Let $f: X \rightarrow \mathbb{S}^{1}$ be a tame map on a compact ANR, and let $\xi=\xi_{f} \in H^{1}(X ; \mathbb{Z})$ denote the corresponding integral cohomology class. Moreover, let $\pi: \tilde{X} \rightarrow X$ denote the associated infinite cyclic covering, that is, the pull back by $f$ of the universal covering $p: \mathbb{R} \rightarrow \mathbb{S}^{1}$. There exists a tame map $\tilde{f}: \tilde{X} \rightarrow \mathbb{R}$ which is equivariant with respect to the (principal) $\mathbb{Z}$-actions on $\tilde{X}$ and $\mathbb{R}$ such that the following diagram commutes:

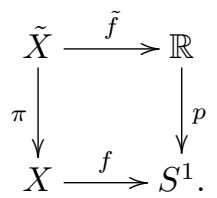

Recall that the vector space $H_{r}(\tilde{X})$ is a $\kappa\left[T^{-1}, T\right]$-modul ${ }^{10}$ where the multiplication by $T$ is the linear isomorphism induced by the fundamental deck transformation $\tau: \tilde{X} \rightarrow \tilde{X}$ corresponding to the action of $1 \in \mathbb{Z}$.

Let $\left.\kappa\left[T^{-1}, T\right]\right]$ be the field of Laurent power series and define

$$
\left.H_{r}^{N}(X ; \xi):=H_{r}(\tilde{X}) \otimes_{\kappa\left[T^{-1}, T\right]} \kappa\left[T^{-1}, T\right]\right] .
$$

The $\left.\kappa\left[T^{-1}, T\right]\right]$-vector spaces $H_{r}^{N}(X ; \xi)$ is called the $r$-th Novikov homology ${ }^{11}$ and its dimension over the field $\left.\kappa\left[T^{-1}, T\right]\right]$, the Novikov-Betti number $\beta_{r}^{N}(X ; \xi)$.

\footnotetext{
${ }^{10} \kappa\left[T^{-1}, T\right]$ denotes the ring of Laurent polynomials with coefficients in $\kappa$, i.e., the group algebra $\kappa[\mathbb{Z}]$.

${ }^{11}$ Instead of $\left.\kappa\left[T^{-1}, T\right]\right]$ one can consider the field $\kappa\left[\left[T^{-1}, T\right]\right.$ of Laurent power series in $T^{-1}$, which is isomorphic to $\left.\kappa\left[T^{-1}, T\right]\right]$ by an isomorphism induced by $T \rightarrow T^{-1}$. The (Novikov) homology defined using this field has the same Novikov-Betti numbers as the one defined using $\left.\kappa\left[T^{-1}, T\right]\right]$.
} 
Consider $H_{r}(\tilde{X}) \rightarrow H_{r}^{N}(X ; \xi)$ the $\kappa\left[T^{-1}, T\right]$-linear map induced by taking the tensor product with $\left.\kappa\left[T^{-1}, T\right]\right]$ over $\kappa\left[T^{-1}, T\right]$. The $\kappa\left[T^{-1}, T\right]$-module $V_{r}(\xi)$,

$$
V_{r}(\xi):=\operatorname{ker}\left(H_{r}(\tilde{X}) \rightarrow H_{r}^{N}(X ; \xi)\right),
$$

when regarded as a $\kappa$-vector space equipped with the linear isomorphism $T_{r}(\xi)$ provided by the multiplication by $T$, is referred to as the $r$-monodromy of $(X, \xi)$. As a $\kappa\left[T^{-1}, T\right]$-module $V_{r}(\xi)$ is exactly the torsion of the $\kappa\left[T^{-1}, T\right]$-module $H_{r}(\tilde{X})$.

A base for $V_{r}(\xi)$ provides a parametrization of the abstract set $\tilde{\mathcal{J}}_{r}(f)$ and therefore an identification of $V_{r}(\xi)$ to $\kappa\left[\tilde{\mathcal{J}}_{r}(f)\right]$. We continue to call "monodromy" and denote by $T_{r}(\xi)$ the isomorphism $T_{r}(\xi): \kappa\left[\tilde{\mathcal{J}}_{r}(f)\right] \rightarrow \kappa\left[\tilde{\mathcal{J}}_{r}(f)\right]$ obtained by using the above identification.

Proof of Theorem 1.1. Part (c) follows from Propositions 3.1 and 2.7 which relate the Betti numbers to the bar codes and Jordan cells via the short exact sequence 19). Similarly one can compute $\operatorname{dim} H_{r}\left(X,\left(\xi_{f}, u\right)\right)$ in terms of barcodes and Jordan cells using the short exact sequence (18).

To check parts (a) and (b) recall that according to Lemma 2.8 we have isomorphisms of $\kappa\left[T^{-1}, T\right]$-modules:

$$
\begin{aligned}
\operatorname{coker} M\left(\tilde{\rho}_{r}(f)\right) & \cong \kappa\left[T^{-1}, T\right]\left[\mathcal{B}_{r}^{c}(f)\right] \oplus \kappa\left[\tilde{\mathcal{J}}_{r}(f)\right], \\
\operatorname{ker} M\left(\tilde{\rho}_{r-1}(f)\right) & \cong \kappa\left[T^{-1}, T\right]\left[\mathcal{B}_{r}^{o}(f)\right] .
\end{aligned}
$$

In particular, the $\kappa\left[T^{-1}, T\right]$-module ker $M\left(\tilde{\rho}_{r-1}(f)\right)$ is free. Hence, the short exact sequence 20 splits and we obtain an isomorphism of $\kappa\left[T^{-1}, T\right]$-modules,

$$
H_{r}(\tilde{X}) \cong \kappa\left[T^{-1}, T\right]\left[\mathcal{B}_{r}^{c}(f) \sqcup \mathcal{B}_{r-1}^{o}(f)\right] \oplus \kappa\left[\tilde{\mathcal{J}}_{r}(f)\right] .
$$

Tensorizing with $\left.\kappa\left[T^{-1}, T\right]\right]$, we obtain an isomorphisms of $\left.\kappa\left[T^{-1}, T\right]\right]$-vector spaces,

$$
\left.H_{r}^{N}\left(X ; \xi_{f}\right) \cong \kappa\left[T^{-1}, T\right]\right]\left[\mathcal{B}_{r}^{c}(f) \sqcup \mathcal{B}_{r-1}^{o}(f)\right] .
$$

We conclude $\beta_{r}^{N}\left(X ; \xi_{f}\right)=\sharp \mathcal{B}_{r}^{c}(f)+\sharp \mathcal{B}_{r-1}^{o}(f)$, whence part (a). Moreover,

$$
V_{r}\left(\xi_{f}\right) \cong \kappa\left[\tilde{\mathcal{J}}_{r}(f)\right] \cong \bigoplus_{J \in \mathcal{J}_{r}(f)} J
$$

whence part (b).

\section{More calculations}

A nonempty subset $K$ of $\mathbb{S}^{1}$ or $\mathbb{R}$, will be called a closed multi-interval if it is a finite union of disjoint closed intervals $\left[\theta_{1}, \theta_{2}\right]$ with $0 \leq \theta_{1} \leq \theta_{2}<2 \pi$ in the case of $\mathbb{S}^{1}$, and $[a, b]$ with $a \leq b$ or $(-\infty, a]$ or $[b, \infty)$ in the case of $\mathbb{R}$. One denotes by $X_{K}:=f^{-1}(K)$ if $K \subset \mathbb{S}^{1}$ and by $\tilde{X}_{K}=f^{-1}(K)$ if $K \subset \mathbb{R}$.

In case $K \subset \mathbb{S}^{1}$ one considers

$$
\begin{aligned}
& \mathcal{B}_{r, K}^{c}(f)=\left\{I \in \mathcal{B}_{r}^{c}(f) \mid I \cap K \neq \emptyset\right\}, \\
& \mathcal{B}_{r, K}^{o}(f)=\left\{I \in \mathcal{B}_{r}^{o}(f) \mid I \subset K\right\},
\end{aligned}
$$

and for $u \in \kappa \backslash 0$ the sets

$$
\begin{aligned}
S_{r, u}(f) & =\mathcal{B}_{r}^{c}(f) \sqcup \mathcal{B}_{r-1}^{o}(f) \sqcup \mathcal{J}_{r, u}(f) \sqcup \mathcal{J}_{r-1, u}(f), \\
S_{r, K, u}(f) & =\mathcal{B}_{r, K}^{c}(f) \sqcup \mathcal{B}_{r-1, K}^{o}(f) \sqcup \mathcal{J}_{r, u}(f) .
\end{aligned}
$$


Recall that $\mathcal{J}_{r, u}(f)$ denotes the set of Jordan blocks $J=(V, T) \in \mathcal{J}_{r}(f)$ whose linear isomorphism $T$ has $u$ as eigenvalue. Since $u \in \kappa \backslash 0, J$ is actually a Jordan cell.

In case $K \subset \mathbb{R}$ an one considers the sets

$$
\begin{aligned}
\widetilde{\mathcal{B}}_{r}(f) & =\left\{I \in \mathcal{B}_{r}(\tilde{f})\right\}, \\
\widetilde{\mathcal{B}}_{r, K}^{c}(f) & =\left\{I \in \mathcal{B}_{r}^{c}(\tilde{f}) \mid I \cap K \neq \emptyset\right\}, \\
\widetilde{\mathcal{B}}_{r, K}^{o}(f) & =\left\{I \in \mathcal{B}_{r}^{o}(\tilde{f}) \mid I \subset K\right\},
\end{aligned}
$$

and

$$
\begin{aligned}
\widetilde{S}_{r, K}(f) & =\widetilde{\mathcal{B}}_{r, K}^{c}(f) \sqcup \widetilde{\mathcal{B}}_{r-1, K}^{o}(f) \sqcup \widetilde{\mathcal{J}}_{r}(f), \\
\widetilde{S}_{r}(f) & =\widetilde{\mathcal{B}}_{r}^{c}(f) \sqcup \widetilde{\mathcal{B}}_{r-1}^{o}(f) \sqcup \widetilde{\mathcal{J}}_{r}(f) .
\end{aligned}
$$

These sets have the following properties:

(i) If $K_{1}, K_{2}, K$ are closed multi-intervals in $\mathbb{S}^{1}$ or $\mathbb{R}$ with $K_{1} \cap K_{2}=\emptyset$ and $K=K_{1} \cup K_{2}$ then $S_{r, K, u}=S_{r, K_{1}, u} \cup S_{r, K_{2}, u}$ and $\tilde{S}_{r, K}=\tilde{S}_{r, K_{1}} \cup \tilde{S}_{r, K_{2}}$.

(ii) If $K_{1}, K_{2}, K$ are closed multi-intervals in $\mathbb{S}^{1}$ or $\mathbb{R}$ with $K_{1} \cap K_{2}=K$ then $S_{r, K, u}=S_{r, K_{1}, u} \cap S_{r, K_{2}, u}$ and $\tilde{S}_{r, K}=\tilde{S}_{r, K_{1}} \cap \tilde{S}_{r, K_{2}}$.

(iii) If $K_{1}, K_{2}$ closed multi-intervals with $K_{1} \subset K_{2}$ then $S_{r, K_{1}, u} \subseteq S_{r, K_{2}, u}$ and $\tilde{S}_{r, K_{1}} \subseteq \tilde{S}_{r, K_{2}}$.

For $K$ a multi-interval in $\mathbb{S}^{1}$ or $\mathbb{R}$ denote by:

$$
\begin{aligned}
\mathbb{I}_{r}(f ; K, u) & :=\operatorname{img}\left(H_{r}\left(X_{K}\right) \rightarrow H_{r}(X ;(\xi, u)),\right. \\
\mathbb{I}_{r}(\tilde{f} ; K) & :=\operatorname{img}\left(H_{r}\left(\tilde{X}_{K}\right) \rightarrow H_{r}(\tilde{X})\right) .
\end{aligned}
$$

Let $f: X \rightarrow \mathbb{S}^{1}$ be a tame map with $m$ critical values $0<\theta_{1}<\theta_{2} \cdots<\theta_{m} \leq 2 \pi$ and $\tilde{f}: \tilde{X} \rightarrow \mathbb{R}$ an infinite cyclic cover of $f$, with critical values $c_{i}, i \in \mathbb{Z}$ with $p\left(c_{i+m k}\right)=\theta_{i}$.

Recall that for a surjective linear map $\pi: A \rightarrow B$ a linear map $s: B \rightarrow A$ such that $\pi \cdot s=i d$ is called a splitting.

For the projections

$$
\begin{aligned}
H_{r}(\tilde{X}) & \rightarrow \operatorname{ker} M\left(\tilde{\rho}_{r-1}(f)\right) \\
H_{r}\left(\tilde{X}_{\left[c_{i}, c_{j}\right]}\right) & \rightarrow \operatorname{ker} M\left(T_{i, j}\left(\tilde{\rho}_{r-1}(f)\right)\right)
\end{aligned}
$$

one considers collections of splittings

$$
\begin{aligned}
& \tilde{s}_{r}: \operatorname{ker} M\left(\tilde{\rho}_{r-1}(f)\right) \rightarrow H_{r}(\tilde{X}) \\
& \tilde{s}_{r ; i, j}: \operatorname{ker} M\left(T_{i, j}\left(\tilde{\rho}_{r-1}(f)\right)\right) \rightarrow H_{r}\left(\tilde{X}_{\left[c_{i}, c_{j}\right]}\right)
\end{aligned}
$$

such that the diagram

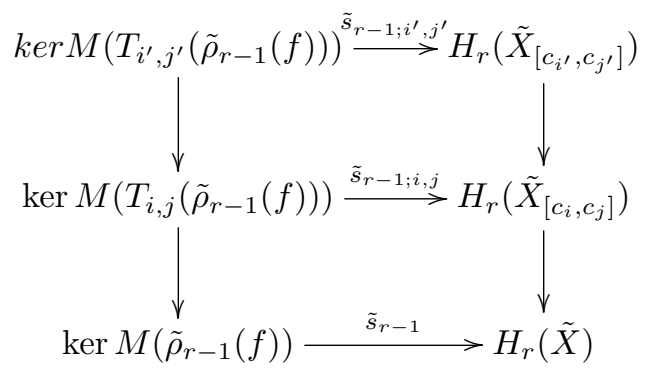


commutes, for all $-\infty<i^{\prime} \leq i \leq j \leq j^{\prime}<\infty$.

A collection of splittings as above is called a collection of compatible splittings. In view of the fact that the splitting $\tilde{s}_{r-1 ; i, j}$ and $\tilde{s}_{r-1 ; j, k}$ can be extended to a splitting $\tilde{s}_{r-1 ; i, k}$, the existence of collections of compatible splittings is straightforward. The construction being realized inductively from $(i, j)$ to $(i, j+1)$ and from $(i, j)$ to $(i-1, j)$. Moreover one can produce collections of compatible splittings which are $m$-periodic, which means that $\tilde{s}_{r}$ intertwines the isomorphism $t_{r}$ with $\tau_{r}$. In other words, $\tilde{s}_{r}$ may be assumed to be a homomorphism of $\kappa\left[T^{-1}, T\right]$-modules

Precisely, we consider the surjective maps $\pi_{r, i, j}: H_{r}\left(\tilde{X}_{\left[c_{i}, c_{j}\right]}\right) \rightarrow \kappa\left[\mathcal{B}^{o}\left(T_{i, j}\left(\tilde{\rho}_{r-1}\right)\right]\right.$, the composition of $H_{r}\left(X_{\left[c_{i}, c_{j}\right]}\right) \rightarrow \operatorname{ker}\left(M\left(T_{i, j}\left(\tilde{\rho}_{r-1}\right)\right)\right.$ with the isomorphism $\left(\Psi_{i, j}^{o}\right)^{-1}$ $\kappa\left[\mathcal{B}^{o}\left(T_{i, j}\left(\tilde{\rho}_{r-1}\right)\right] \rightarrow \operatorname{ker}\left(M\left(T_{i, j}\left(\tilde{\rho}_{r-1}\right)\right)\right.\right.$. Here $\tilde{\rho}_{r-1}$ abbreviates $\tilde{\rho}_{r-1}(f)$. For any bar code $I \in B^{o}\left(\tilde{\rho}_{r-1}\right)$ with ends $c_{i}$ and $c_{j}$ call lift of $I$ an element $v_{I} \in H_{r}\left(\tilde{X}_{\left[c_{i}, c_{j}\right]}\right)$ s.t. $\pi_{r, i, j}\left(v_{I}\right)=I$.

It is straightforward to provide a family of lifts $v_{I}$ for any $I \in \mathcal{B}^{o}\left(\rho_{r-1}\right)$ s.t. in view of surjectivity of $\pi_{r, i, j}$ all $v_{I}$ with $I$ of the same ends linearly independent and in view of the isomorphism $t^{*}: H_{r}\left(\tilde{X}_{\left[c_{i}, c_{j}\right]}\right) \rightarrow H_{r}\left(\tilde{X}_{\left[c_{i+m}, c_{j+m}\right]}\right), c_{i+m}=c_{i}+2 \pi$, with $v_{I}$ satisfying $v_{I+2 \pi}=t^{*}\left(v_{I}\right)$. Define $\bar{s}_{r-1, i, j}: \kappa\left[\mathcal{B}^{o}\left(T_{i, j}\left(\tilde{\rho}_{r-1}\right) \rightarrow H_{r}\left(\tilde{X}_{\left[c_{i}, c_{j}\right]}\right.\right.\right.$ and $\bar{s}_{r-1}: \kappa\left[\mathcal{B}^{o}\left(\tilde{\rho}_{r-1}\right) \rightarrow H_{r}(\tilde{X})\right.$ by taking $\bar{s}_{r-1, i, j}(I)$ to be the image of $v_{I}$ in $H_{r}\left(\tilde{X}_{\left[c_{i}, c_{j}\right]}\right.$ and $\tilde{s}_{r-1}\left(\Psi^{o}(I)\right.$ to be the image of $v_{I}$ in $H_{r}(\tilde{X})$. Define $\tilde{s}_{r-1, i, j}=\bar{s}_{r-1, i, j} \cdot \Psi_{i, j}^{o}$ and $\tilde{s}_{r-1}=\bar{s}_{r-1} \cdot \Psi^{o}$.

A similar definition can be obtained by replacing $\tilde{\rho}_{r-1}(f)$ and $H_{r}(\tilde{X})$ by $\rho_{r-1}(f)_{u}$ and $H_{r}\left(X ;\left(\xi_{f}, u\right)\right)$.

With the notations and the definitions above we have the following technical results which calculate $\mathbb{I}_{r}(f ; K, u)$ and $\mathbb{I}_{r}(\tilde{f} ; K)$ as well as homologies of $H_{r}\left(X,\left(\xi_{f}, u\right)\right)$, $H_{r}(\tilde{X})$ already described.

Proposition 4.1. Let $f: X \rightarrow \mathbb{S}^{1}$ be a tame map and suppose that for each $r$ a decomposition of the representation $\rho_{r}(f)$ as a sum of bar code representations and Jordan block and a collection of compatible splittings is provided. Then:

a) For $u \in \kappa \backslash 0$ the decompositions and the collections of compatible splittings provide the isomorphisms

$$
\omega_{r, u}: \kappa\left[S_{r, u}(f)\right] \rightarrow H_{r}\left(X ;\left(\xi_{f}, u\right)\right)
$$

and for any closed multi interval $K \subset \mathbb{S}^{1}$ the isomorphisms

$$
\omega_{r, K, u}: \kappa\left[S_{r, K, u}(f)\right] \rightarrow \mathbb{I}_{r}(f ; K, u)
$$

such that for any pair $K^{\prime}, K$ of closed multi-intervals in $\mathbb{S}^{1}$ with $K^{\prime} \subset K$, the diagram (30) is commutative.

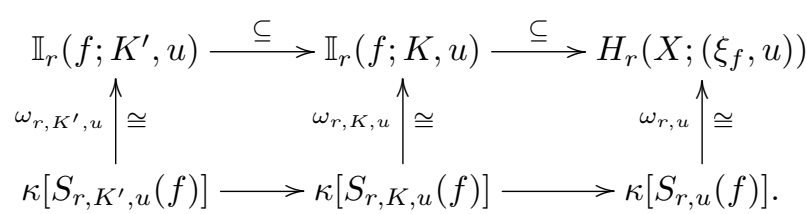

The horizontal arrows in the bottom line are induced by the inclusions of the sets in brackets.

b) The decompositions and the collection of compatible splittings provide the isomorphisms

$$
\widetilde{\omega}_{r}: \kappa\left[\widetilde{S}_{r}(f)\right] \rightarrow H_{r}(\tilde{X})
$$


and for any closed multi interval $K \subset \mathbb{R}$ the isomorphisms

$$
\widetilde{\omega}_{r, K}: \kappa\left[\widetilde{S}_{r, K}(f)\right] \rightarrow \mathbb{I}_{r}(\tilde{f} ; K)
$$

such that for any pair $K^{\prime}, K$ of closed multi-intervals in $\mathbb{R}$ with $K^{\prime} \subset K$, the diagram (31) is commutative.

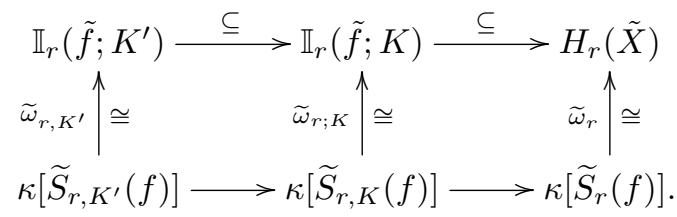

The horizontal arrows in the bottom line are induced by the inclusions of the sets in brackets. The isomorphism $\tilde{\omega}_{r}$ is an isomorphism of $\kappa\left[T^{-1}, T\right]$-modules.

c) The decompositions and the splittings provide the isomorphisms

$$
\left.\omega_{r}^{N}: \kappa\left[T^{-1}, T\right]\right]\left[\mathcal{B}_{r}^{c}(f) \sqcup \mathcal{B}_{r-1}^{o}(f)\right] \rightarrow H_{r}^{N}\left(X ; \xi_{f}\right) .
$$

\section{Even more calculations}

It is also possible to calculate $H_{r}\left(X_{K}\right)$ for $K \subset \mathbb{S}^{1}$ and $H_{r}\left(\tilde{X}_{K}\right)$ for $K \subset \mathbb{R}$. In this case, in addition to closed and open bar codes and to Jordan blocks, the mixed bar codes will appear.

For the purpose of definition below we treat a closed interval $K^{\prime}=\left[\theta^{\prime}, \theta^{\prime \prime}\right] \subset \mathbb{S}^{1}$, $0<\theta^{\prime} \leq \theta^{\prime \prime}<2 \pi$ as the closed interval $K=\left[\theta^{\prime}, \theta^{\prime \prime}\right] \subset \mathbb{R}$

To formulate the result for $K$ a closed interval of $\mathbb{R}$ we add to the previous definitions, see formulae (26), the sets:

$$
\begin{gathered}
\widetilde{\mathcal{B}}_{r, K}^{c o}(f)=\left\{I \in \widetilde{\mathcal{B}}_{r}^{c o}(f) \mid I \cap K \neq \emptyset \text { and closed }\right\}, \\
\widetilde{\mathcal{B}}_{r, K}^{o c}(f)=\left\{I \in \widetilde{\mathcal{B}}_{r}^{o c}(f) \mid I \cap K \neq \emptyset \text { and closed }\right\} \\
\widetilde{\mathcal{B}}_{r, K}^{o o}(f)=\left\{I \in \widetilde{\mathcal{B}}_{r}^{o}(f) \mid I \supset K\right\}
\end{gathered}
$$

and denote by $\widetilde{S}_{r, K}^{\prime}(f)$ the set

$$
\widetilde{S}_{r, K}^{\prime}(f)=\widetilde{\mathcal{B}}_{r, K}^{c o}(f) \sqcup \widetilde{\mathcal{B}}_{r, K}^{o c}(f) \sqcup \widetilde{\mathcal{B}}_{r, K}^{o o}(f) \sqcup \widetilde{S}_{r, K} .
$$

We have $\widetilde{S}_{r, K}(f) \subseteq \widetilde{S}_{r, K}^{\prime}(f)$.

Proposition 4.2. a) The decompositions and the collections of compatible splittings provide for any pair of angles $\theta^{\prime}, \theta^{\prime \prime}, 0<\theta^{\prime} \leq \theta^{\prime \prime}<2 \pi$, the isomorphisms

$$
\omega_{r,\left[\theta^{\prime}, \theta^{\prime \prime}\right]}^{\prime}: \kappa\left[S_{r,\left[\theta^{\prime}, \theta^{\prime \prime}\right]}^{\prime}(f)\right] \rightarrow H_{r}\left(X_{\left[\theta^{\prime}, \theta^{\prime \prime}\right]}\right)
$$

such that for $0<\theta_{1} \leq \theta_{2} \leq \theta_{3} \leq \theta_{4}<2 \pi$ the diagram (33) below is commutative:

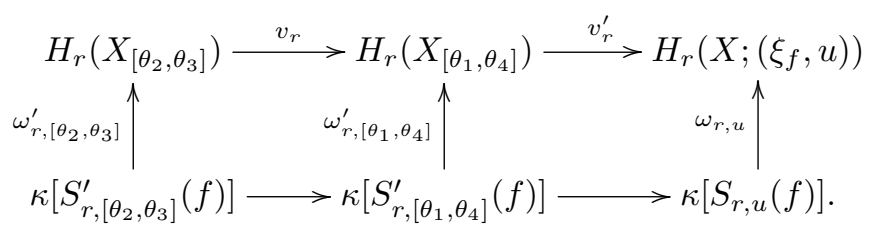

b) The decompositions and the compatible splittings provide for any $a \leq b, a, b$ real numbers or $\pm \infty$ the isomorphisms

$$
\tilde{\omega}_{r,[a, b]}^{\prime}: \kappa\left[\tilde{S}_{r,[a, b]}^{\prime}(f)\right] \rightarrow H_{r}\left(\tilde{X}_{[a, b]}\right)
$$


such that for $a \leq b \leq c \leq d$ the diagram (34) below is commutative.

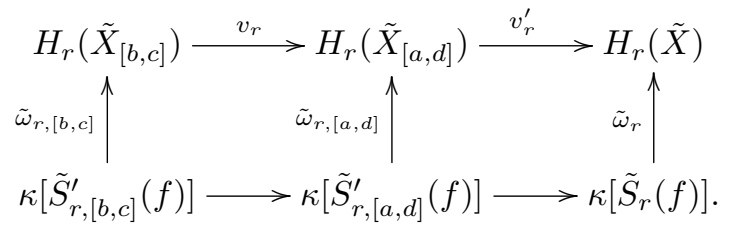

In both diagrams the horizontal arrows in the top line are linear maps induced by the obvious inclusions, while in the bottom line are the canonical linear maps provided by the sets in brackets subsets of a larger set of all bar codes and all $\tilde{\mathcal{J}}(f)^{\prime} s, c f$. Definition 2.6. A bar code in the set $S_{r, \ldots}^{\prime}$ or in $\tilde{S}_{r, \ldots}^{\prime}$ is sent to itself if belongs to the next set and if not, to zero in the next vector space.

Proposition 4.2 permits to express the vector spaces $H_{r}\left(\tilde{X}_{[a, b]}\right), H_{r}\left(\tilde{X}_{[c, d]} \backslash \tilde{X}_{(a, b)}\right)$ and the linear maps $H_{r}\left(\tilde{X}_{[a, b]}\right) \rightarrow H_{r}\left(\tilde{X}_{[c, d]}\right)$ and $H_{r}\left(\tilde{X}_{[c, d]} \backslash \tilde{X}_{(a, b)}\right) \rightarrow H_{r}\left(\tilde{X}_{[c, d]}\right)$ in terms of the bar codes $\tilde{\mathcal{B}}_{-}^{-}(f)$ and $\tilde{\mathcal{J}}_{-}(f)$. This will be used in section 66 .

Proof of Propositions 4.1 and 4.2. In view of the properties of the sets $S_{K,-}$ and $\tilde{S}_{K,-}$, it suffices to prove the statements for $K$ consisting of one single interval and in view the tameness of $f$ one can suppose that $\theta_{1}, \theta_{2}$ are critical angles and $a, b$ critical values. We treat first the part (a) in both Propositions (4.1) and (4.2).

The compatible splittings lead to the commutative diagram (35) with horizontal arrows isomorphisms.

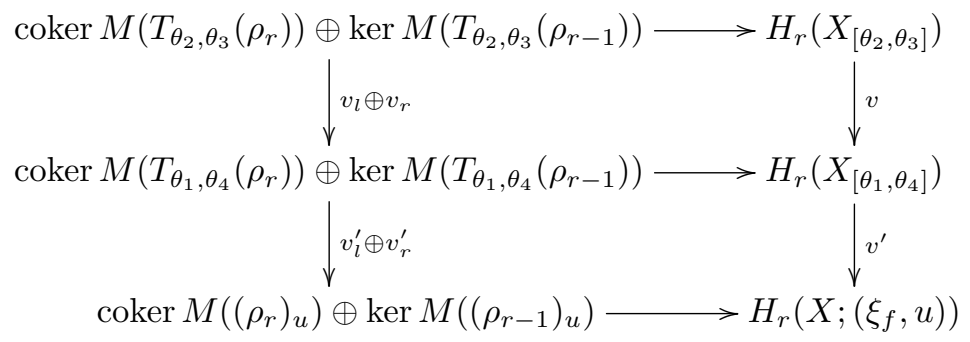

Proposition 2.7 combined with Lemma 2.2 gives the commutative diagram (36) with horizontal lines isomorphisms.

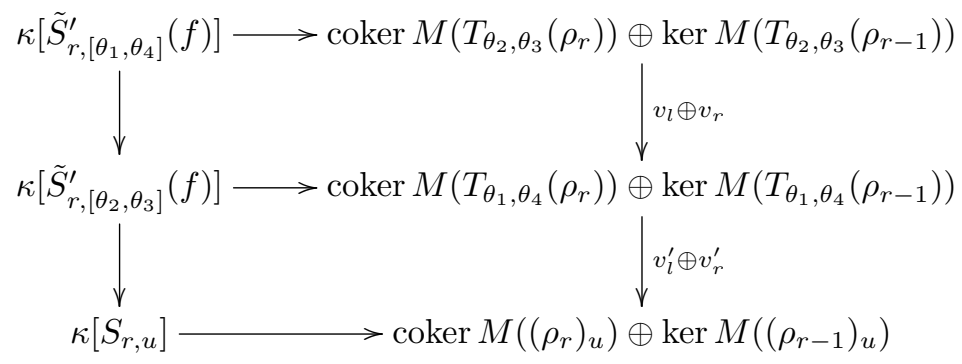

The isomorphism $\omega_{r, u}$ (in Proposition 4.1) is the composition of horizontal arrows in the last line of diagrams (35) and (36) and the isomorphisms $\omega_{r,\left[\theta_{2}, \theta_{3}\right], u}$ and $\omega_{r,\left[\theta_{1}, \theta_{4}\right], u}$ are restrictions of $\omega_{r, u}$. Similarly he isomorphism $\omega_{r,\left[\theta_{2}, \theta_{3}\right]}^{\prime}$ and $\omega_{r,\left[\theta_{1}, \theta_{4}\right]}^{\prime}$ (in Proposition 4.2) are the compositions of the horizontal arrows in the first and second lines of the same diagrams. The commutativity of the diagrams 30 and 
(33) is the consequence of the commutativity of the diagrams (35) and (36). This establishes part (a) in both Propositions 4.1 and 4.2

Parts (b) are verified essentially in the same way. More precisely, the decompositions of the representations $\rho_{r}$ imply decompositions of $\tilde{\rho}_{r}$ and $T_{k, l}\left(\tilde{\rho}_{r}\right)$. Observe that the commutative diagrams (35) and (36) remain valid when one replaces $X$ by $\tilde{X}$, the representation $\rho_{r}$ by $\tilde{\rho}_{r}$, and $\theta_{1}, \theta_{2}, \theta_{3}, \theta_{4}$ by $a, b, c, d$. In this case $\tilde{\omega}$ is defined in the same way as $\omega_{u}$, namely as the composition of the horizontal arrows in the last lines of the diagrams which replace diagrams (35) and (36) derived considering $\tilde{\omega}$ instead of $\omega_{u}$.

To check part (c) in Proposition 4.1, observe first that $\kappa\left[\tilde{S}_{r}(f)\right]=\kappa\left[\tilde{\mathcal{B}}_{r-1}^{o}(f)\right] \oplus$ $\kappa\left[\tilde{\mathcal{B}}_{r}^{c}(f) \sqcup \tilde{\mathcal{J}}_{r}(f)\right]$ and as pointed out by Lemma 2.8 at the end of Section 2 , both linear maps $\Psi^{o}$ and $\Psi^{c}$ are actually isomorphisms of $\kappa\left[T^{-1}, T\right]$ modules; therefore so is $\tilde{\omega}_{r}$. Then one takes $\left.\omega_{r}^{N}=\tilde{\omega}_{r} \otimes_{\kappa\left[T^{-1}, T\right]} \kappa\left[T^{-1}, T\right]\right]$. Clearly $\kappa\left[\tilde{S}_{r}(f)\right] \otimes_{\kappa\left[T^{-1}, T\right]}$ $\left.\left.\kappa\left[T^{-1}, T\right]\right]=\kappa\left[T^{-1}, T\right]\right]\left[\mathcal{B}_{r}^{c}(f) \sqcup \mathcal{B}_{r-1}^{o}(f)\right]$ since $\kappa[\tilde{J}(f)]$ as a $\kappa\left[T^{-1}, T\right]$-module is a torsion module, cf. Lemma 2.8 .

\section{Stability for configurations $C_{r}(f)$. Proof of Theorem 1.2}

The proof of Theorems 1.2 and 1.3 will require an alternative definition of the configurations $C_{r}(f)$. This will be provided by the integer valued functions $\delta_{r}^{f}$ which will be defined for a proper real-valued tame map and then, via the infinite cyclic covering for a tame angle-valued map. Ultimately they are defined for any continuous map.

5.1. Real valued maps. For $f: X \rightarrow \mathbb{R}$ a map and $a, b \in \mathbb{R}$, introduce the notation $X^{f}(a)=f^{-1}(a), X_{a}^{f}=f^{-1}((-\infty, a]), X_{f}^{b}=f^{-1}([b, \infty)),{ }^{f} X_{a}^{b}=X_{f}^{a} \cap X_{b}^{f}$. Let $i_{a}^{f}: X_{a}^{f} \rightarrow X$ and $i_{f}^{b}: X_{f}^{b} \rightarrow X$ be the obvious inclusions. Denote by

$$
\begin{aligned}
& \mathbb{I}_{a}^{f}(r):=\operatorname{img}\left(i_{a}^{f}(r): H_{r}\left(X_{a}^{f}\right) \rightarrow H_{r}(X)\right), \\
& \mathbb{I}_{f}^{b}(r):=\operatorname{img}\left(i_{f}^{b}(r): H_{r}\left(X_{f}^{b}\right) \rightarrow H_{r}(X)\right),
\end{aligned}
$$

and let $F_{r}^{f}(a, b):=\operatorname{dim}\left(\mathbb{I}_{a}^{f}(r) \cap \mathbb{I}_{f}^{b}(r)\right)$ and $G_{r}^{f}(a, b):=\operatorname{dim} H_{r}(X) /\left(\mathbb{I}_{a}^{f}(r)+\mathbb{I}_{f}^{b}(r)\right)$.

Observe that:

\section{Lemma 5.1.}

(a) For $a \leq a^{\prime}$ and $b^{\prime} \leq b$, we have $F_{r}^{f}(a, b) \leq F_{r}^{f}\left(a^{\prime}, b^{\prime}\right)$ and $G_{r}^{f}(a, b) \geq G_{r}^{f}\left(a^{\prime}, b^{\prime}\right)$.

(b) If $|f-g|<\epsilon$ and $a \leq b$ then $F_{r}^{f}(a-\epsilon, b+\epsilon) \leq F_{r}^{g}(a, b)$ and $G_{r}^{f}(a, b) \leq$ $G_{r}^{g}(a-\epsilon, b+\epsilon)$.

(c) $F_{r}^{f}(a, b)=F_{r}^{-f}(-b,-a)$ and $G_{r}^{f}(a, b)=G_{r}^{-f}(-b,-a)$.

Proof. To check (a), notice that $X_{a}^{f} \subseteq X_{a^{\prime}}^{f}$ and $X_{f}^{b^{\prime}} \supseteq X_{f}^{b}$ imply $\mathbb{I}_{a}^{f} \subseteq \mathbb{I}_{a^{\prime}}^{f}$ and $\mathbb{I}_{f}^{b^{\prime}} \subseteq \mathbb{I}_{f}^{b}$ and $\mathbb{I}_{f}^{b^{\prime}} \subseteq \mathbb{I}_{f}^{b}$, hence $\mathbb{I}_{a}^{f} \cap \mathbb{I}_{f}^{b} \subseteq \mathbb{I}_{a^{\prime}}^{f} \cap \mathbb{I}_{f}^{b^{\prime}}$ and $\mathbb{I}_{a}^{f}+\mathbb{I}_{f}^{b} \subseteq \mathbb{I}_{a^{\prime}}^{f}+\mathbb{I}_{f}^{b^{\prime}}$, then the statement. To check (b), notice that $|f-g|<\epsilon$ implies $f-\epsilon<g<f+\epsilon$ which implies $X_{a-\epsilon}^{f} \subseteq X_{a}^{g}$ and $X_{b+\epsilon}^{f} \subseteq X_{b}^{g}$. These inclusions imply $\mathbb{I}_{a-\epsilon}^{f} \subseteq \mathbb{I}_{a}^{g}$ and $\mathbb{I}_{f}^{b+\epsilon} \subseteq \mathbb{I}_{g}^{b}$, hence $F^{f}(a-\epsilon, b+\epsilon) \leq F^{g}(a, b)$. The arguments for $G$ are similar. To check (c), one uses the fact that $f^{-1}((-\infty, a])=(-f)^{-1}([-a, \infty))$.

If $X$ is a compact ANR it is immediate that both $F_{r}^{f}(a, b)$ and $G_{r}^{f}(a, b)$ are finite since $\operatorname{dim} H_{r}(X)$ is finite. The same remains true for $f: X \rightarrow \mathbb{R}$ a tame 
map (hence proper) with $X$ not compact despite the fact that $\operatorname{dim} H_{r}(X)$ is not necessarily finite.

Proposition 5.2. If $f: X \rightarrow \mathbb{R}$ is a tame map, then:

(a) $F_{r}^{f}(a, b)<\infty$.

(b) $G_{r}^{f}(a, b)<\infty$.

Proof. To ease the writing, we (sometimes) drop $f$ from notation. We start with (a). In view of Observation 5.1 it suffices to check the statements for $a>b$.

Consider

$$
i_{a}(r)-i^{b}(r): H_{r}\left(X_{a}\right) \oplus H_{r}\left(X^{b}\right) \rightarrow H_{r}(X),
$$

let $p_{1}: H_{r}\left(X_{a}\right) \oplus H_{r}\left(X^{b}\right) \rightarrow H_{r}\left(X_{a}\right)$ be the first factor projection and observe that $\mathbb{I}_{a}^{f}(r) \cap \mathbb{I}_{f}^{b}(r)=\left(i_{a}(r) \cdot p_{1}\right)\left(\operatorname{ker}\left(i_{a}(r)-i^{b}(r)\right)\right.$.

Then

$$
\operatorname{dim}\left(\mathbb{I}_{a}^{f}(r) \cap \mathbb{I}_{f}^{b}(r)\right) \leq \operatorname{dim} \operatorname{ker}\left(i_{a}(r)-i^{b}(r)\right) .
$$

Since $a \geq b$ we have $X=X_{a} \cup X^{b}$. In view of the Mayer-Vietoris long exact sequence associated with $X=X_{a} \cup X^{b}$

$$
\operatorname{ker}\left(i_{a}(r)-i^{b}(r)\right)=\operatorname{img}\left(H_{r}\left(X_{a}^{b}\right) \rightarrow H_{r}\left(X_{a}\right) \oplus H_{r}\left(X^{b}\right)\right)
$$

has finite dimension since $\operatorname{dim} H_{r}\left(X_{a}^{b}\right)$ is finite.

Next we prove (b). If $a<b$ one uses the long exact sequence of the pair $\left(X, X_{a} \sqcup X^{b}\right)$ to conclude that $H_{r}(X) /\left(\mathbb{I}_{a}^{f}(r)+\mathbb{I}_{f}^{b}(r)\right)$ is isomorphic to a subspace of $H_{r}\left(X, X_{a} \sqcup X^{b}\right)=H_{r}\left(X_{a}^{b}, X(a) \sqcup X(b)\right)$ which is of finite dimension. Indeed, $f$ tame implies that $X(a), X(b)$, and $X_{a}^{b}$ are compact ANRs, hence with finite dimensional homology.

If $a \geq b$ one uses the Mayer-Vietoris exact sequence associated with $X_{a}, X^{b}$ to conclude that $H_{r}(X) /\left(\mathbb{I}_{a}^{f}(r)+\mathbb{I}_{f}^{b}(r)\right)$ is isomorphic to a subspace of $H_{r}\left(X_{a}^{b}\right)$ which is of finite dimension.

Let $a<b$ and $c<d$. We refer to the set

$$
B(a, b: c, d)=(a, b] \times[c, d) \subset \mathbb{R}^{2}, \quad a<b, c<d,
$$

as a "box", and define:

$$
\begin{aligned}
& \mu_{r}^{F, f}(B)=F_{r}^{f}(a, d)+F_{r}^{f}(b, c)-F_{r}^{f}(a, c)-F_{r}^{f}(b, d), \\
& \mu_{r}^{G, f}(B)=-G_{r}^{f}(a, d)-G_{r}^{f}(b, c)+G_{r}^{f}(a, c)+G_{r}^{f}(b, d) .
\end{aligned}
$$

One has:

Proposition 5.3. If $X$ is compact or $f$ is a tame map, then:

(a) $\mu_{r}^{F, f}(B)=\mu_{r}^{G, f}(B)$.

(b) Putting $\mu_{r}^{f}(B):=\mu_{r}^{F, f}(B)=\mu_{r}^{G, f}(B)$, we have $\mu_{r}^{f}(B) \geq 0$.

(c) If $B=B_{1} \cup B_{2}, B_{1} \cap B_{2}=\emptyset$ with $B_{1}, B_{2}$ boxes, then $\mu^{f}(B)=\mu^{f}\left(B_{1}\right)+\mu^{f}\left(B_{2}\right)$.

(d) If if $B^{\prime}$ and $B^{\prime \prime}$ are boxes with $B^{\prime} \subseteq B^{\prime \prime}$ one has $\mu^{f}\left(B^{\prime}\right) \leq \mu^{f}\left(B^{\prime \prime}\right)$. 


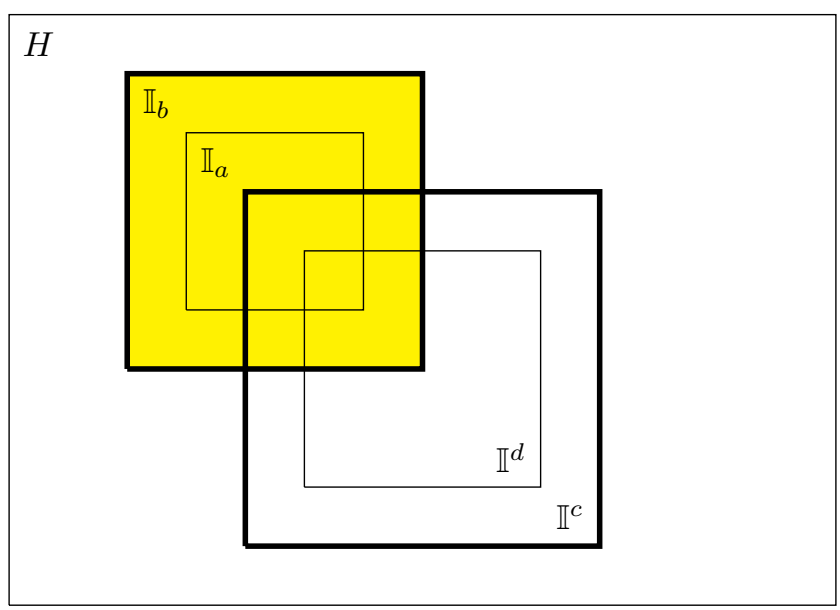

FiguRE 4. An illustration for the proof of Proposition 5.3 .

Proof. To ease the writing, we drop $f$ and $r$ from notation and introduce:

$$
\begin{aligned}
& I_{1}:=\operatorname{dim}\left(\mathbb{I}_{a} \cap \mathbb{I}^{d}\right), \\
& I_{2}:=\operatorname{dim}\left(\left(\mathbb{I}_{a} \cap \mathbb{I}^{c}\right) /\left(\mathbb{I}_{a} \cap \mathbb{I}^{d}\right)\right), \\
& I_{3}:=\operatorname{dim}\left(\left(\mathbb{I}_{b} \cap \mathbb{I}^{d}\right) /\left(\mathbb{I}_{a} \cap \mathbb{I}^{d}\right)\right), \\
& I_{4}:=\operatorname{dim}\left(\left(\mathbb{I}_{b} \cap \mathbb{I}^{c}\right) /\left(\mathbb{I}_{a} \cap \mathbb{I}^{c}+\mathbb{I}_{b} \cap \mathbb{I}^{d}\right)\right), \\
& I_{5}:=\operatorname{dim}\left(\mathbb{I}_{b} /\left(\mathbb{I}_{a}+\mathbb{I}_{b} \cap \mathbb{I}^{c}\right)\right), \\
& I_{6}:=\operatorname{dim}\left(\mathbb{I}^{c} /\left(\mathbb{I}_{a} \cap \mathbb{I}^{c}+\mathbb{I}^{d}\right)\right), \\
& I_{7}:=\operatorname{dim}\left(H /\left(\mathbb{I}_{b}+\mathbb{I}^{c}\right)\right), \quad \text { with } H=H_{r}(X) .
\end{aligned}
$$

Using Figure 4, it is not hard to notice that:

$$
\begin{aligned}
F(a, d) & =I_{1}, & G(a, d) & =I_{7}+I_{6}+I_{5}+I_{4}, \\
F(b, c) & =I_{1}+I_{2}+I_{3}+I_{4}, & G(b, c) & =I_{7}, \\
F(a, c) & =I_{1}+I_{2}, & G(a, c) & =I_{7}+I_{5}, \\
F(b, d) & =I_{1}+I_{3}, & G(b, d) & =I_{7}+I_{6} .
\end{aligned}
$$

Then we have:

$$
\begin{aligned}
F(a, d)+F(b, c)-F(a, c)-F(b, d) & \\
& =I_{1}+\left(I_{1}+I_{2}+I_{3}+I_{4}\right)-\left(I_{1}+I_{2}\right)-\left(I_{1}+I_{3}\right)=I_{4}
\end{aligned}
$$

and

$$
\begin{aligned}
G(a, d)+G(b, c)-G(a, c)-G(b, d) & \\
& =\left(I_{7}+I_{6}+I_{5}+I_{4}\right)+I_{7}-\left(I_{7}+I_{5}\right)-\left(I_{7}+I_{6}\right)=I_{4} .
\end{aligned}
$$

These equalities establish (a) and (b). Part (c) follows from (37) by inspecting the all relative positions of $B_{1}$ and $B_{2}$ as disjoint subsets of $B$. To check part (d) one tiles $B^{\prime \prime}$ as a disjoint union of boxes $B^{\prime \prime}=B_{1}^{\prime \prime} \sqcup B_{2}^{\prime \prime} \cdots \sqcup B_{r-1}^{\prime \prime} \sqcup B_{r}^{\prime \prime}$ s.t. $B^{\prime}$ is one 
of these boxes and use item (c) to inductively derive that $\mu\left(B^{\prime \prime}\right)=\sum_{1 \leq i \leq r} \mu\left(B_{u}^{\prime \prime}\right)$. This implies the result

Note that both Propositions (5.2) and (5.3) remain valid for $f$ a proper continuous map but with more elaborated arguments and this is not not needed in this paper.

Define the jump function, $\delta_{r}^{f}: \mathbb{R}^{2} \rightarrow Z_{\geq 0}$, by

$$
\delta_{r}^{f}(a, b):=\lim _{\epsilon \rightarrow 0} \mu^{f}((a-\epsilon, a+\epsilon] \times[b-\epsilon, b+\epsilon)) .
$$

The limit exists since, by Proposition 5.3 (c), the right side decreases when $\epsilon$ decreases. This function has values in $\mathbb{Z}_{\geq 0}$. Since the critical values of a tame map are discrete, $\delta_{r}^{f}$ has discrete support and satisfies the following proposition.

Proposition 5.4. If $X$ is compact or $f$ is a tame map then:

(a) For $a<b$ and $c<d$ one has $\mu_{r}^{f}((a, b] \times[c, d))=\sum_{a<x \leq b, c \leq y<d} \delta_{r}^{f}(x, y)$.

(b) $F_{r}^{f}(b, c)=\sum_{x \leq b, c \leq y} \delta_{r}^{f}(x, y)$.

(c) $G_{r}^{f}(a, d)=\sum_{a \leq x, y \leq d} \delta_{r}^{f}(x, y)$.

Proof. Item (a) follows from Proposition 5.3 c) as shown below.

First observe that in in view of $(37)$ if both $(b-a)$ and $(d-c)$ are small enough then $\mu_{r}^{f}((a, b] \times[c, d))=\delta_{r}^{f}(c, d)$. Then choose subdivisions $a_{0}=a<a_{1}<\cdots<$ $a_{r}=b$ and $c=c_{0}<c_{1}<\cdots<c_{k}=d$ such that all critical values between $a$ and $b$ rep. between $c$ and $d$ are among $a_{i}$ 's and $c_{j}$ 's respectively and both $a_{i}-a_{i-1}$ and $c_{j}-c_{j-1}$ are small enough s.t. $\mu_{r}^{f}\left(\left(a_{i-1}, a_{i}\right] \times\left[c_{i}, c_{i+1}\right)\right)=\delta_{r}^{f}\left(a_{i}, c_{i}\right)$. Applying Proposition 5.3 c one obtains $\mu_{r}^{f}((a, b] \times[c, d))=\sum_{i \geq 1, j \geq 1} \mu_{r}^{f}\left(\left(a_{i-1}, a_{i}\right] \times\left[c_{j-1}, c_{j}, d\right)\right)$ which implies the result as stated.

Item (b) follows from (a) by letting $a \rightarrow-\infty$ and $d \rightarrow \infty$. Similarly, item (c) follows from item (a) by letting $b \rightarrow \infty$ and $c \rightarrow-\infty$.

Since for a tame map $f$ the set of critical values is discrete we write them as a sequence $\cdots<c_{i-1}<c_{i}<c_{i+1}<\cdots$ and define

$$
\epsilon(f)=\inf _{i \in \mathbb{Z}}\left(c_{i+1}-c_{i}\right) .
$$

Clearly, if $f: X \rightarrow \mathbb{R}$ is tame with $X$ compact, then $\epsilon(f)>0$ and if $f: X \rightarrow \mathbb{S}^{1}$ is tame then the infinite cyclic covering $\tilde{f}: \tilde{X} \rightarrow \mathbb{R}$ is tame and $\epsilon(\tilde{f})>0$.

Proposition 5.5. Let $f: X \rightarrow \mathbb{R}$ be a tame map with $\epsilon(f)>0$. For any $\epsilon, \epsilon^{\prime}<\epsilon(f)$ one has:

$$
F_{r}^{f}\left(c_{i}, c_{j}\right)=F_{r}^{f}\left(c_{i}+\epsilon, c_{j}-\epsilon^{\prime}\right)=F_{r}^{f}\left(c_{i+1}-\epsilon, c_{j-1}+\epsilon^{\prime}\right),
$$

and

$$
\delta_{r}^{f}\left(c_{i}, c_{j}\right)=F_{r}^{f}\left(c_{i-1}, c_{j+1}\right)+F_{r}^{f}\left(c_{i}, c_{j}\right)-F_{r}^{f}\left(c_{i-}, c_{j}\right)-F_{r}^{f}\left(c_{i}, c_{j+1}\right) .
$$

Proof. The tameness of $f$ and the hypothesis $\epsilon, \epsilon^{\prime}<\epsilon(f)$ imply that the inclusions $X_{c_{i}}^{f} \subseteq X_{c_{i}+\epsilon}^{f}, X_{c_{i}}^{f} \subseteq X_{c_{i+1}-\epsilon^{\prime}}^{f}$ and $X_{f}^{c_{j}-\epsilon} \supseteq X_{f}^{c_{j}}, X_{f}^{c_{j-1}+\epsilon^{\prime}} \supseteq X_{f}^{c_{j}}$ induce isomorphisms in homology. These facts imply that $\mathbb{I}_{c_{i}}^{f}=\mathbb{I}_{c_{i}+\epsilon}^{f}=\mathbb{I}_{c_{i+1}-\epsilon^{\prime}}^{f}$ and $\mathbb{I}_{f}^{c_{j-1}+\epsilon}=\mathbb{I}_{f}^{c_{j}-\epsilon^{\prime}}=\mathbb{I}_{f}^{c_{j}}$ which imply (39). To check (40), recall that in view of 37), (38) and 39, for $\epsilon$ very small, one has $\delta_{r}^{f}\left(c_{i}, c_{j}\right)=F_{r}\left(c_{i}-\epsilon, c_{j}+\epsilon\right)+F_{r}\left(c_{i}+\epsilon, c_{j}-\right.$ 
$\epsilon)-F_{r}\left(c_{i}-\epsilon, c_{j}-\epsilon\right)-F_{r}\left(c_{i}+\epsilon, c_{j}+\epsilon\right)$. Thus 40 follows from 39 by taking $\epsilon<\epsilon(f)$.

For a pair $(a, b) \in \mathbb{R}^{2}$ and $\epsilon>0$ consider the box $B(a, b ; 2 \epsilon)=(a-2 \epsilon, a+2 \epsilon] \times$ $[b-2 \epsilon, b+2 \epsilon)$.

Proposition 5.6. Let $f: X \rightarrow \mathbb{R}$ be a tame map with $\epsilon(f)>0$ and $\epsilon<\epsilon(f) / 4$. For any tame map $g$ with $|f-g|<\epsilon$ and any $(a, b) \in \operatorname{supp} \delta_{r}^{f}$ the following holds:

(a) $\operatorname{supp}\left(\delta_{r}^{f}\right) \cap B(a, b ; 2 \epsilon) \equiv(a, b)$

(b) $\sharp\left(\operatorname{supp}\left(\delta_{r}^{g}\right) \cap\left(\bigsqcup_{(a, b) \in \operatorname{supp} \delta_{r}^{f}} B(a, b ; 2 \epsilon)\right)\right)=\sharp \operatorname{supp}\left(\delta_{r}^{f}\right)$.

In particular, if the cardinality of the support $t^{12}$ of $\delta_{r}^{f}$ and $\delta_{r}^{g}$ are equal and $|g-f|<$ $\epsilon$, then the support of $\delta_{r}^{g}$ lies in an $\epsilon$-neighborhood ${ }^{13}$ of the support of $\delta_{r}^{f}$.

This Proposition is closed to Box Lemma in [11] page 112.

Proof. Item (a) follows from the definition of $\delta_{r}^{f}$. To prove item (b) observe that if $(a, b) \in \operatorname{supp} \delta$ both numbers have to be critical values, hence the $a=c_{i}, b=c_{j}$. In view of Proposition 5.5. for any $\epsilon^{\prime}, \epsilon^{\prime \prime}<\epsilon(f)$ one has:

$$
\begin{aligned}
F_{r}^{f}\left(c_{i-1}, c_{j+1}\right) & =F_{r}^{f}\left(a-\epsilon^{\prime}, b+\epsilon^{\prime \prime}\right) \\
F_{r}^{f}\left(c_{i}, c_{j}\right) & =F_{r}^{f}\left(a+\epsilon^{\prime}, b-\epsilon^{\prime \prime}\right) \\
F_{r}^{f}\left(c_{i}, c_{j+1}\right) & =F_{r}^{f}\left(a+\epsilon^{\prime}, b+\epsilon^{\prime \prime}\right) \\
F_{r}^{f}\left(c_{i-1}, c_{j}\right) & =F_{r}^{f}\left(a-\epsilon^{\prime}, b-\epsilon^{\prime \prime}\right)
\end{aligned}
$$

Since $|f-g|<\epsilon$, in view of Observation 5.1 one has:

$$
\begin{aligned}
& F_{r}^{f}(a-3 \epsilon, b+3 \epsilon) \leq F_{r}^{g}(a-2 \epsilon, b+2 \epsilon) \leq F_{r}^{f}(a-\epsilon, b+\epsilon) \\
& F_{r}^{f}(a+\epsilon, b-\epsilon) \leq F_{r}^{g}(a+2 \epsilon, b-2 \epsilon) \leq F_{r}^{f}(a+3 \epsilon, b-3 \epsilon) \\
& F_{r}^{f}(a+\epsilon, b+3 \epsilon) \leq F_{r}^{g}(a+2 \epsilon, b+2 \epsilon) \leq F_{r}^{f}(a+3 \epsilon, b+\epsilon) \\
& F_{r}^{f}(a-3 \epsilon, b-\epsilon) \leq F_{r}^{g}(a-2 \epsilon, b-2 \epsilon) \leq F_{r}^{f}(a-\epsilon, b-3 \epsilon)
\end{aligned}
$$

Since $\epsilon<\epsilon(f) / 4$, equations (41) and (42) imply:

$$
\begin{aligned}
& F_{r}^{g}(a-2 \epsilon, b+2 \epsilon)=F_{r}^{f}\left(c_{i-1}, c_{j+1}\right) \\
& F_{r}^{g}(a+2 \epsilon, b-2 \epsilon)=F_{r}^{f}\left(c_{i}, c_{j}\right) \\
& F_{r}^{g}(a+2 \epsilon, b+2 \epsilon)=F_{r}^{f}\left(c_{i}, c_{j+1}\right) \\
& F_{r}^{g}(a-2 \epsilon, b-2 \epsilon)=F_{r}^{f}\left(c_{i-1}, c_{j}\right)
\end{aligned}
$$

In view of Proposition 5.4 we have

$$
\begin{aligned}
\sharp\left(\operatorname{supp}\left(\delta_{r}^{g}\right) \cap B(a, b ; 2 \epsilon)\right)= & \mu_{r}^{g}(B(a, b ; 2 \epsilon)) \\
= & F_{r}^{g}(a-2 \epsilon, b+2 \epsilon)+F_{r}^{g}(a+2 \epsilon, b-2 \epsilon) \\
& -F_{r}^{g}(a-2 \epsilon, b-2 \epsilon)-F_{r}^{g}(a+2 \epsilon, b+2 \epsilon) .
\end{aligned}
$$

Using the equations 43 and the equation 40 in Proposition 5.5 one derives

$$
\sharp\left(\operatorname{supp}\left(\delta_{r}^{g}\right) \cap B(a, b ; 2 \epsilon)\right)=\sharp\left(\operatorname{supp}\left(\delta_{r}^{f}\right) \cap B(a, b ; 2 \epsilon)\right)=\delta_{r}^{f}(a, b),
$$

\footnotetext{
${ }^{12}$ Recall that the cardinality of the support is the sum of multiplicity of the elements in the support.

${ }^{13}$ Here $\epsilon$-neighborhood of $(a, b)$ means the domain $(a-\epsilon, a+\epsilon) \times(b-\epsilon, b+\epsilon)$.
} 
5.2. Angle valued maps. Let $f: X \rightarrow \mathbb{S}^{1}$ be a tame map and $\tilde{f}: \tilde{X} \rightarrow \mathbb{R}$ its infinite cyclic covering. Note that $\epsilon(\tilde{f})>0$. Observe that

$$
\delta_{r}^{\tilde{f}}(a, b)=\delta_{r}^{\tilde{f}}(a+2 \pi, b+2 \pi) .
$$

Consider the projection $p: \mathbb{R}^{2} \rightarrow \mathbb{T}=\mathbb{R}^{2} / \mathbb{Z}$, with $\mathbb{T}$ the quotient space of $\mathbb{R}^{2}$ by the action $\mathbb{Z} \times \mathbb{R}^{2} \rightarrow \mathbb{R}^{2}$ given by $(n,(a, b)) \rightarrow(a+2 \pi n, b+2 \pi n)$. Write $p(a, b)=\langle a, b\rangle$. Define

$$
\epsilon(f):=\epsilon(\tilde{f})
$$

and

$$
\delta_{r}^{f}(\langle a, b\rangle):=\delta_{r}^{\tilde{f}}(a, b) .
$$

In view of (44), $\delta_{r}^{f}: \mathbb{T} \rightarrow \mathbb{Z}_{\geq 0}$ is a well defined function with finite support and Proposition 5.6 holds true for $f: X \rightarrow \mathbb{S}^{1}$.

For the proof of Theorem 1.2 we also need to show that $\delta_{r}^{f}$ and $C_{r}(f)$, when viewed as functions on $\mathbb{T}$, are equal.

Proposition 5.7. If $f$ is a tame real-or angle-valued map defined on $X$, a compact $A N R$, then $\delta_{r}^{f}$ and $C_{r}(f)$ are equal $\mathbb{Z}_{\geq 0}$-valued functions defined on $\mathbb{R}^{2}$ or $\mathbb{T}$.

Proof. We check the case of an angle valued map $f: X \rightarrow \mathbb{S}^{1}$ only. The real valued case can be regarded as a particular case of the angle valued map. First note that $\epsilon(f)>0$. In view of the definition of $\delta_{r}^{\tilde{f}}$ it suffices to check that:

(i) If at least one, $a$ or $b$, is not a critical value then we have $\delta_{r}^{\tilde{f}}(a, b)=0$.

(ii) If $a=c_{i}$ and $b=c_{j}$ are critical values with $c_{i} \geq c_{j}$, then

$$
\delta_{r}^{\tilde{f}}\left(c_{i}, c_{j}\right)=\sharp\left\{I \in \tilde{\mathcal{B}}_{r}^{c}(f) \mid I=\left[c_{j}, c_{i}\right]\right\} .
$$

(iii) If $a=c_{i}$ and $b=c_{j}$ are critical values with $c_{i}<c_{j}$, then

$$
\delta_{r}^{\tilde{f}}\left(c_{i}, c_{j}\right)=\sharp\left\{I \in \tilde{\mathcal{B}}_{r-1}^{o}(f) \mid I=\left(c_{j}, c_{j}\right)\right\} .
$$

Recall that $\delta_{r}^{\tilde{f}}(a, b)=\lim _{\epsilon \rightarrow 0}\left(-F_{r}^{\tilde{f}}(a-\epsilon, b-\epsilon)-F_{r}^{\tilde{f}}(a+\epsilon, b+\epsilon)+F_{r}^{\tilde{f}}(a-\epsilon, b+\right.$ $\left.\epsilon)+F_{r}^{\tilde{f}}(a+\epsilon, b-\epsilon)\right)$. In view of Proposition 5.5. if $a$ is not a critical value, $y \in \mathbb{R}$ and $\epsilon>0$ is sufficiently small, then $F_{r}^{\tilde{f}}(a-\epsilon, y)=F_{r}^{\tilde{f}}(a+\epsilon, y)$ and thus $\delta_{r}^{\tilde{f}}(a, y)=0$. Similarly, if $b$ is not a critical value, $x \in \mathbb{R}$ and $\epsilon>0$ is sufficiently small, then $F_{r}^{\tilde{f}}(x, b-\epsilon)=F_{r}^{\tilde{f}}(x, b+\epsilon)$ and thus $\delta_{r}^{\tilde{f}}(x, b)=0$. This establishes statement (i).

Suppose that $a=c_{i}$ and $b=c_{j}$ critical values. In view of Proposition 5.5 and of the definition of $\delta^{\tilde{f}}$ one has

$$
\delta_{r}^{\tilde{f}}\left(c_{i}, c_{j}\right)=-F_{r}^{\tilde{f}}\left(c_{i-1}, c_{j}\right)-F_{r}^{\tilde{f}}\left(c_{i}, c_{j+1}\right)+F_{r}^{\tilde{f}}\left(c_{i-1}, c_{j+1}\right)+F_{r}^{\tilde{f}}\left(c_{i}, c_{j}\right) .
$$

By Propositions 4.2 (b) when $c_{i} \geq c_{j}$, one has

$$
\begin{aligned}
F_{r}^{\tilde{f}}\left(c_{i}, c_{j}\right)=\sharp\left\{I \in \tilde{\mathcal{B}}_{r}^{c}(f) \mid I \cap\left[c_{j}, c_{i}\right]\right. & \neq \emptyset\} \\
+ & \sharp\left\{I \in \tilde{\mathcal{B}}_{r-1}^{o}(f) \mid I \subset\left(c_{j}, c_{i}\right)\right\}+\sharp \tilde{\mathcal{J}}_{r}(f),
\end{aligned}
$$

and when $c_{i}>c_{j}$ one has

$$
F_{r}^{\tilde{f}}\left(c_{i}, c_{j}\right)=\sharp\left\{I \in \tilde{\mathcal{B}}_{r}^{c}(f) \mid I \supset\left[c_{i}, c_{j}\right]\right\}+\sharp \tilde{\mathcal{J}}_{r}(f) .
$$


Indeed Proposition 4.2 (b) calculates $\mathbb{I}_{a}^{\tilde{f}}(r)$ and $\mathbb{I}_{\tilde{f}}^{b}(r)$ as the $\kappa$-vector space generated by

$$
\left\{I \in \widetilde{\mathcal{B}}_{r}^{c} \mid I \cap(-\infty, a] \neq \emptyset\right\} \cup\left\{I \in \widetilde{\mathcal{B}}_{r-1}^{o} \mid I \subset(-\infty, a)\right\}
$$

and

$$
\left\{I \in \widetilde{\mathcal{B}}_{r}^{c} \mid I \cap([b, \infty) \neq \emptyset\} \cup\left\{I \in \widetilde{\mathcal{B}}_{r-1}^{o} \mid I \subset(b, \infty)\right\}\right.
$$

of which the above descriptions follow.

Comparing the collections of bar codes whose cardinality are given by $F_{r}^{\tilde{f}}\left(c_{i-1}, c_{j}\right)$, $F_{r}^{\tilde{f}}\left(c_{i}, c_{j+1}\right), F_{r}^{\tilde{f}}\left(c_{i-1}, c_{j+1}\right)$ and $F_{r}^{\tilde{f}}\left(c_{i}, c_{j}\right)$ and using (46), (47) and 48) one derives the statement (ii), and (iii).

To prove Theorem 1.2 one begins with a few observations.

(i) Consider the space of continuous maps $C\left(X, \mathbb{S}^{1}\right), X$ a compact ANR, with the compact open topology. This topology is induced from the metric $D(f, g):=$ $\sup _{x \in X} d(f(x), g(x))$ with $d\left(\theta_{1}, \theta_{2}\right)$ given by $d\left(\theta_{1}, \theta_{2}\right)=\inf \left(\left|\theta_{1}-\theta_{2}\right|, 2 \pi-\mid \theta_{1}-\right.$ $\left.\theta_{2} \mid\right), 0 \leq \theta_{1}, \theta_{2}<2 \pi$. Equipped with this metric $\left(C\left(X, \mathbb{S}^{1}\right), D\right)$ is a complete metric space.

Recall that the set of connected components of the space $C\left(X, \mathbb{S}^{1}\right)$ identifies to $H^{1}(X ; \mathbb{Z})$. Denote by $C_{\xi}\left(X, \mathbb{S}^{1}\right)$ the connected component corresponding to the class $\xi \in H^{1}(X ; \mathbb{Z})$ and by $C_{\xi, t}\left(X, \mathbb{S}^{1}\right)$ the subset of tame maps in this connected component equipped with the induced topology.

(ii) Observe that if $f, g$ are in a connected component $C_{\xi}\left(X, \mathbb{S}^{1}\right)$ of $C\left(X, \mathbb{S}^{1}\right)$ and $D(f, g)<\pi$ then for any $t \in[0,1]$ the map $h_{t}:=h_{t}(f, g) \in C\left(X ; \mathbb{S}^{1}\right)$ defined below lies in the connected component of $C_{\xi}\left(X, \mathbb{S}^{1}\right)$. Moreover for any $0=$ $t_{0}<t_{1}<\cdots<t_{N-1}<t_{N}=1$ one has

$$
D(f, g)=\sum_{0 \leq i<N} D\left(h_{t_{i+1}}, h_{t_{i}}\right) .
$$

Considering the inclusion of $\mathbb{S}^{1} \subset \mathbb{R}^{2}$ as the unit circle centered at origin, if one regards $f$ and $g$ as $\mathbb{R}^{2}$-valued maps, the map $h_{t}$ is defined by

$$
h_{t}(x)=\frac{t f(x)+(1-t) g(x)}{\|t f(x)+(1-t) g(x)\|} .
$$

(iii) Recall that $f$ is a p.l. map on $X$ if with respect to some subdivision is simplicial (i.e. the liftings to $\mathbb{R}$ of the restriction of $f$ to simplexes of the subdivision are linear) and for any two p.l. maps $f, g$ there exists a common subdivision of $X$ which makes $f$ and $g$ simultaneously simplicial, hence any $h_{t}$ is a simplicial map.

If $X$ is a simplicial complex and $\mathcal{U} \subset C_{\xi}\left(X, \mathbb{S}^{1}\right)$ denotes the subset of p.l. maps then:

(1) $\mathcal{U}$ is a dense subset in $C_{\xi}\left(X, \mathbb{S}^{1}\right)$.

(2) $f, g \in \mathcal{U}$ implies $h_{t} \in \mathcal{U}$ hence $\epsilon\left(h_{t}\right)>0$ hence for any $t \in[0,1]$ there exists $o(t)>0$ so that $\left|t^{\prime}-t\right|<o(t)$ implies $D\left(h_{t^{\prime}}, h_{t}\right)<\epsilon\left(h_{t}\right) / 6$.

Indeed (1) follows from approximability of continuous maps by p.l. maps and (2) from the continuity in $t$ of the family $h_{t}$ and the compacity of $X$.

(iv) For $k$ a positive integer consider $S^{k} \mathbb{T}=(\mathbb{T} \times \cdots \times \mathbb{T}) / \Sigma_{k}$, with $\Sigma_{k}$ the $k$ symmetric group acting on the $k$-fold cartesian product of $\mathbb{T}$ by permutations equipped with the metric $\underline{D}$ induced from the complete metric on $\mathbb{T}=\mathbb{R}^{2} / \mathbb{Z}$. With this metric $\left(S^{N}(\mathbb{T}), \underline{D}\right)$ is a complete metric space. 
(v) Proposition 5.6 states that $f, g \in C\left(X, \mathbb{S}^{1}\right)_{t, \xi}$ and $D(f, g)<\epsilon(f) / 6$ implies

$$
\underline{D}\left(\delta_{r}^{f}, \delta_{r}^{g}\right)<2 D(f, g) \text {. }
$$

\section{Proof of Theorem 1.2:}

Observation $\mathrm{v}$ makes the assignment $C\left(X, \mathbb{S}^{1}\right)_{t, \xi} \ni f \mapsto \delta_{r}^{f} \in S_{r}^{\beta_{r}^{N}(X, \xi)}(\mathbb{T})$ a continuous map.

In order to conclude the existence of a continuous extension of $\delta_{r}^{f}$ to the entire $C_{\xi}\left(X, \mathbb{S}^{1}\right)$, in view of the completeness of the metrics $D$, and $\underline{D}$, stated in observations (ii) and (iv) above, it suffices to show that for a Cauchy sequence $\left\{f_{\alpha}\right\}, f_{\alpha} \in \mathcal{U}$, the sequence $\delta_{r}^{f_{\alpha}}$ is a Cauchy sequence in $S^{\beta_{r}^{N}(X, \xi)}(\mathbb{T})$. This will follow once we can show that (50) holds for any two $f, g \in \mathcal{U}$ with $d(f, g)<\pi$. To establish this we proceed as in [11, Section 3.3].

Start with $f, g \in \mathcal{U}$ with $D(f, g)<\pi$ and consider $h_{t}, t \in[0,1]$ defined in (ii) above. Choose a finite sequence $0=t_{0}<t_{2}<t_{4}<\cdots<t_{2 L-2}<t_{2 L}=1, L$ positive integer, so that the open intervals $I_{2 i}=\left(t_{2 i}-o\left(t_{2 i}\right), t_{2 i}+o\left(t_{2 i}\right)\right)$ cover $[0,1]$ with $o(t)$ from item (iii) (2). The compacity of $[0,1]$ makes such choice possible.

By possibly removing some of the points $t_{2 i}$ and decreasing $o\left(t_{2 i}\right)$ if necessary one can make $I_{2 i} \cap I_{2 i+2} \neq \emptyset$ and $t_{2 t_{-} 2}, t_{2 i+2} \notin I_{2 i}$. Choose $t_{1}<t_{3}<\cdots<t_{2 L-1}$ with $t_{2 i}<t_{2 i+1}<t_{2 i}$ and $t_{2 i+1} \in I_{2 i} \cap I_{2 i+2}$. We have then $\left|t_{2 i+1}-t_{2 i}\right|<o\left(t_{2 i}\right)$ and $\left|t_{2 i+2}-t_{2 i+1}\right|<o\left(t_{2 i+2}\right)$.

In view of item (iii), $\left|t_{2 i+1}-t_{2 i}\right|<o\left(t_{2 i}\right)$ implies

$$
D\left(h_{t_{2 i}}, h_{t_{2 i+1}}\right)<\epsilon\left(h_{t_{2 i}}\right) / 3
$$

and $\left|t_{2 i+2}-t_{2 i+1}\right|<o\left(t_{2 i+2}\right)$ implies

$$
D\left(h_{t_{2 i+2}}, h_{t_{2 i+1}}\right)<\epsilon\left(h_{t_{2 i+2}}\right) / 6 \text {. }
$$

In view of item $\sqrt{\mathrm{V}}$ the last inequalities imply

$$
\underline{D}\left(\delta_{r}^{h_{t_{2 i+1}}}, \delta_{r}^{h_{t_{2 i}}}\right)<2 D\left(h_{t_{2 i}}, h_{t_{2 i+1}}\right)
$$

as well as

$$
\underline{D}\left(\delta_{r}^{h_{t_{2 i+2}}}, \delta_{r}^{h_{t_{2 i+1}}}\right)<2 D\left(h_{t_{2 i+2}}, h_{t_{2 i+1}}\right) .
$$

Therefore, for any $0 \leq k \leq 2 L-1$ one has $\underline{D}\left(\delta_{r}^{h_{t_{k+1}}}, \delta_{r}^{h_{t_{k}}}\right)<2 D\left(h_{t_{k+1}}, h_{t_{k}}\right)$. Then by (50) and 49) cf item (ii), one obtains

$$
\underline{D}\left(\delta^{f}, \delta^{g}\right) \leq \sum_{0 \leq i<2 L-1} \underline{D}\left(\delta^{h_{t_{i+1}}}, \delta^{h_{t_{i}}}\right) \leq 2 \sum_{0 \leq i<2 L-1} D\left(h_{t_{i+1}}, h_{t_{i}}\right)=D(f, g) .
$$

This finishes the proof of Theorem 1.2 .

\section{Poincaré duality for configurations $C_{r}(f)$. Proof of Theorem 1.3}

For an $n$-dimensional manifold, not necessarily compact, Poincaré duality can be better formulated using Borel-Moore homology, cf. [9], especially tailored for locally compact spaces $Y$ and pairs $(Y, K), K$ closed subset of $Y$. Borel-Moore homology coincides with the standard homology when $Y$ is compact. In general, for a locally compact space $Y$, it can be described as the inverse limit of the homology vector spaces $H_{r}(Y, Y \backslash U)$ for all $U$ open sets with compact closure.

One denotes by $H_{r}^{\mathrm{BM}}(\cdots)$ the Borel-Moore homology in dimension $r$. For $Y$ an $n$-dimensional topological $\kappa$-orientable manifold, $g: Y \rightarrow \mathbb{R}$ a tame map, hence a 
proper continuous map, and $a$ a regular value of $g{ }^{14}$ Poincaré duality provides the commutative diagrams

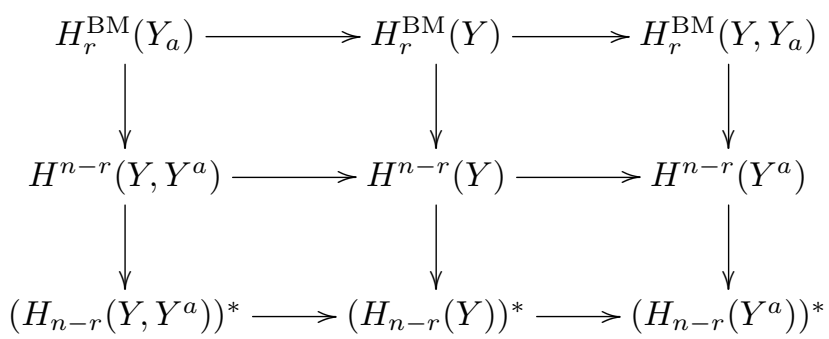

and

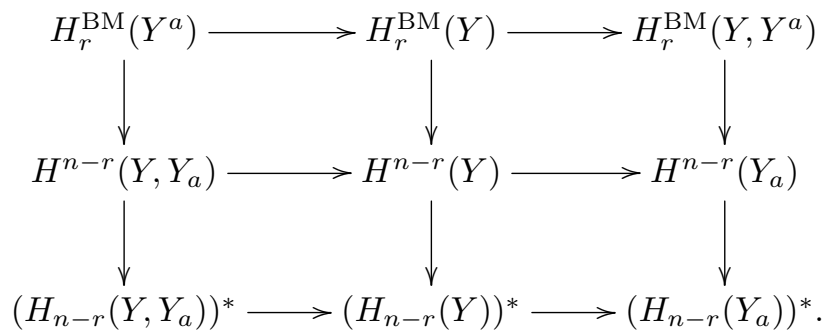

Recall that $Y_{a}=f^{-1}((-\infty, a])$ and $Y^{a}=f^{-1}([a, \infty))$. The first vertical arrow in each column of the diagrams (51) and (52) is the Poincaré duality isomorphism, the second is the isomorphism between cohomology and the dual of homology with coefficients in a field. The horizontal arrows are induced by the inclusions of $Y_{a}$ or $Y^{a}$ in $Y$ and the inclusion of the pairs $(Y, \emptyset)$ in $\left(Y, Y_{a}\right)$ or $\left(Y, Y^{a}\right)$.

We apply diagrams (51) and (52) to $Y=\tilde{M}$ and $g=\tilde{f}$, where $\tilde{M}$ is an infinite cyclic cover of $M$ defined by $f: M \rightarrow \mathbb{S}^{1}$, a tame map, $M$ a closed $\kappa$-orientable topological manifold, and $\tilde{f}: \tilde{M} \rightarrow \mathbb{R}$ the lift of $f$ to $\tilde{M}$. One obtains the commutative diagrams

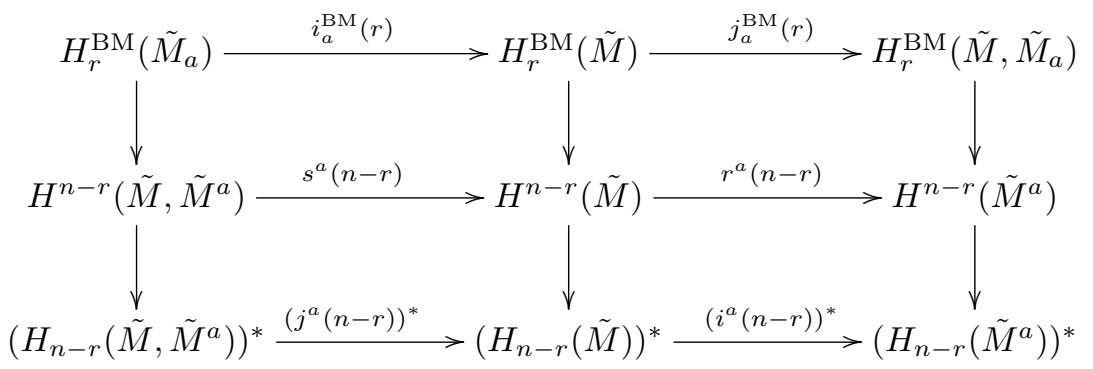

\footnotetext{
14. i.e. $f: f^{-1}(a-\epsilon, a+\epsilon) \rightarrow(a-\epsilon, a+\epsilon)$ is a fibration
} 
and

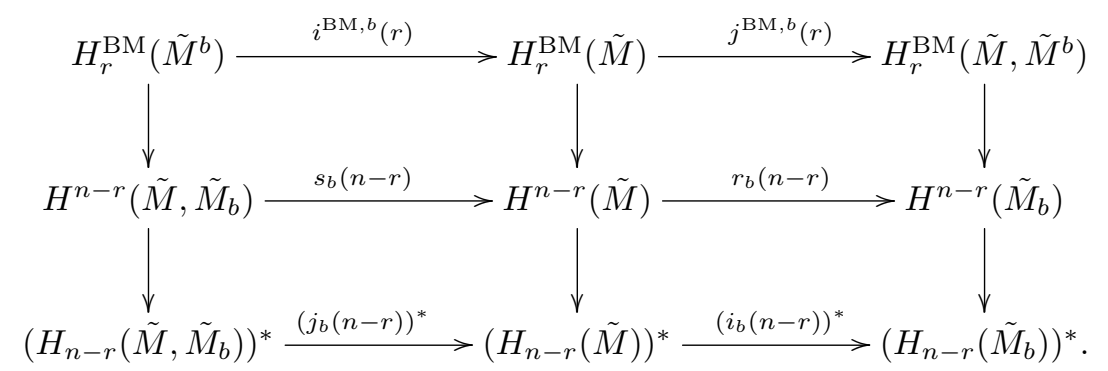

For $\tilde{M}, \tilde{M}_{a}$, and $\tilde{M}^{a}$ the Borel-Moore homology can be described as the following inverse limits:

$$
\begin{aligned}
H_{r}^{\mathrm{BM}}(\tilde{M}) & =\lim _{0<l \rightarrow \infty} H_{r}\left(\tilde{M}, \tilde{M}_{-l} \sqcup \tilde{M}^{l}\right), \\
H_{r}^{\mathrm{BM}}\left(\tilde{M}_{a}\right) & =\lim _{0<t \rightarrow \infty} H_{r}\left(\tilde{M}_{a}, \tilde{M}_{a-l}\right), \\
H_{r}^{\mathrm{BM}}\left(\tilde{M}^{a}\right) & =\lim _{0<l \rightarrow \infty} H_{r}\left(\tilde{M}^{a}, \tilde{M}^{a+l}\right),
\end{aligned}
$$

The inclusions of pairs $\left(\tilde{M}, \tilde{M}_{-l^{\prime}} \sqcup \tilde{M}^{l^{\prime}}\right) \subseteq\left(\tilde{M}, \tilde{M}_{-l} \sqcup \tilde{M}^{l}\right)$ for $l^{\prime}>l$ induce in homology an inverse system whose limit is $H_{r}^{\mathrm{BM}}(\tilde{M})$. Similar inclusions of pairs associated with $l^{\prime}>l$ induce inverse systems whose limits are the remaining BorelMoore homology vector spaces considered above.

The horizontal arrows in both diagrams are inclusion induced linear maps in Borel-Moore homology, cohomology and homology.

In view of the use of Borel-Moore homology, in addition to $\mathbb{I}_{a}^{\tilde{f}}(r)$ and $\mathbb{I}_{\tilde{f}}^{a}(r)$, one considers

$$
\begin{aligned}
& \mathbb{I}_{a}^{\mathrm{BM}, \tilde{f}}(r)=\operatorname{img}\left(H_{r}^{\mathrm{BM}}\left(\tilde{M}_{a}\right) \rightarrow H_{r}^{\mathrm{BM}}(\tilde{M})\right), \\
& \mathbb{I}_{\tilde{f}}^{\mathrm{BM}, a}(r)=\operatorname{img}\left(H_{r}^{\mathrm{BM}}\left(\tilde{M}^{a}\right) \rightarrow H_{r}^{\mathrm{BM}}(\tilde{M})\right),
\end{aligned}
$$

and $F_{r}^{\mathrm{BM}, f}(a, b)=\operatorname{dim}\left(\mathbb{I}_{a}^{\mathrm{BM}, \tilde{f}}(r) \cap \mathbb{I}_{\tilde{f}}^{\mathrm{BM}, b}(r)\right)$.

The first step in the proof of Theorem 1.3 is the verification of the equality

$$
F_{r}^{B M, \tilde{f}}(a, b)=G_{n-r}^{f}(b, a),
$$

the second the verification of the equality

$$
F_{r}^{B M, \tilde{f}}(a, b)+\sharp \tilde{\mathcal{J}}_{r}(f)=F_{r}^{\tilde{f}}(a, b)
$$

and the third step the verification of the equality

$$
\delta_{n-r}^{\tilde{f}}(b, a)=\delta_{r}^{\tilde{f}}(a, b) .
$$

In view of the definition of $\delta_{r}^{f}(\langle a, b\rangle)$, cf. 45, , the last equality implies $\delta_{r}^{f}(\langle a, b\rangle)=$ $\delta_{n-r}^{f}(\langle b, a\rangle)$ hence Theorem 1.3 .

STEP 1:

Recall that if $\alpha^{\prime}: A^{\prime} \rightarrow B$ and $\alpha^{\prime \prime}: A^{\prime \prime} \rightarrow B$ are linear maps, one writes $\alpha^{\prime}+\alpha^{\prime \prime}$ for the linear map $\alpha^{\prime}+\alpha^{\prime \prime}: A^{\prime} \oplus A^{\prime \prime} \rightarrow B$ defined by

$$
\left(\alpha^{\prime}+\alpha^{\prime \prime}\right)\left(a^{\prime}, a^{\prime \prime}\right):=\alpha\left(a^{\prime}\right)+\alpha^{\prime \prime}\left(a^{\prime \prime}\right)
$$


and if $\beta^{\prime}: A \rightarrow B^{\prime}$ and $\beta^{\prime \prime}: A \rightarrow B^{\prime \prime}$ are linear maps, one writes $\left(\beta^{\prime}, \beta^{\prime \prime}\right)$ for the linear map $\left(\beta^{\prime}, \beta^{\prime \prime}\right): A \rightarrow B^{\prime} \oplus B^{\prime \prime}$ defined by

$$
\left(\beta^{\prime}, \beta^{\prime \prime}\right)(a):=\left(\beta^{\prime}(a), \beta^{\prime \prime}(a)\right) .
$$

One has the canonical isomorphisms

$$
\begin{gathered}
\operatorname{ker}\left(\beta^{\prime}, \beta^{\prime \prime}\right)^{*} \simeq \operatorname{coker}\left(\left(\beta^{\prime}\right)^{*}+\left(\beta^{\prime \prime}\right)^{*}\right) \\
\operatorname{ker}\left(\alpha^{\prime}+\alpha^{\prime \prime}\right)^{*} \simeq \operatorname{coker}\left(\left(\alpha^{\prime}\right)^{*},\left(\alpha^{\prime \prime}\right)^{*}\right) .
\end{gathered}
$$

The exact sequences in Borel-Moore homology of the pairs $\left(\tilde{M}, \tilde{M}_{a}\right)$ and $\left(\tilde{M}, \tilde{M}^{b}\right)$, which are the top horizontal rows of the two diagrams (53) and (54), imply

$$
F_{r}^{\mathrm{BM}, \tilde{f}}(a, b)=\operatorname{dim}\left(\mathbb{I}_{a}^{\mathrm{BM}, \tilde{f}}(r) \cap \mathbb{I}_{\tilde{f}}^{\mathrm{BM}, b}(r)\right)=\operatorname{dim} \operatorname{ker}\left(j_{a}^{\mathrm{BM}}(r), j^{\mathrm{BM}, b}(r)\right) .
$$

Looking to the right side corners of the diagrams (53) and (54) one concludes

$$
\operatorname{ker}\left(j_{a}^{\mathrm{BM}}(r), j^{\mathrm{BM}, b}(r)\right) \equiv \operatorname{ker}\left(r^{a}(n-r), r_{b}(n-r)\right) .
$$

In view of the canonical isomorphism between cohomology vector space and the dual of homology vector space one obtains:

$$
\operatorname{ker}\left(r^{a}(n-r), r_{b}(n-r)\right) \equiv\left(\operatorname{coker}\left(i^{a}(n-r)+i_{b}(n-r)\right)\right)^{*} .
$$

Observe that:

(1) In view of the finite dimensionality of $G^{\tilde{f}}(a, b)$ one has

$$
\operatorname{dim} G_{n-r}^{\tilde{f}}(b, a)=\operatorname{dim} G_{n-r}^{\tilde{f}}(b, a)^{*} .
$$

(2) In view of the definition of $G_{n-r}^{f}$ one has

$$
\operatorname{dim} G_{n-r}^{\tilde{f}}(b, a)^{*}=\operatorname{dim} \operatorname{coker}\left(i_{b}(n-r)+i^{a}(n-r)\right)^{*} .
$$

(3) In view of (56) one has

$$
\operatorname{coker}\left(i_{b}(n-r)+i^{a}(n-r)\right)^{*}=\operatorname{dim}\left(\operatorname{ker}\left(i_{b}(n-r)^{*}+i^{a}(n-r) *\right)\right)^{*}
$$

(4) In view of (58) and (59) one has

$$
\operatorname{dim} \operatorname{coker}\left(i_{b}(n-r)+i^{a}(n-r)\right)^{*}=\operatorname{dim}\left(\operatorname{ker}\left(j^{B M, b}(r), j_{a}^{B M}(r)\right) .\right.
$$

(5) In view of (57) one has

$$
\operatorname{dim}\left(\operatorname{ker}\left(j^{B M, b}(r), j_{a}^{B M}(r)\right)=F_{r}^{B M, f}(a, b) .\right.
$$

Consequently, $F_{r}^{\mathrm{BM}, \tilde{f}}(a, b)=G_{n-r}^{\tilde{f}}(b, a)$.

\section{STEP 2:}

We first provide below the description of the Borel-Moore homologies considered above in terms of subsets of $\tilde{\mathcal{B}}_{r}(f) \sqcup \tilde{\mathcal{B}}_{r-1}(f) \sqcup \tilde{\mathcal{J}}_{r}(f) \sqcup \tilde{\mathcal{J}}_{r-1}(f)$. This is a little more than we need but is useful for future references.

For $\alpha<\beta$, we use the notations

$$
\begin{aligned}
& i_{\alpha, \beta}(r): H_{r}\left(\tilde{M}_{\alpha}\right) \rightarrow H_{r}\left(\tilde{M}_{\beta}\right) \\
& i^{\alpha, \beta}(r): H_{r}\left(\tilde{M}^{\beta}\right) \rightarrow H_{r}\left(\tilde{M}^{\alpha}\right) \\
& i_{\alpha}^{\beta}(r): H_{r}\left(\tilde{M}_{\alpha} \sqcup \tilde{M}^{\beta}\right) \rightarrow H_{r}(\tilde{M})
\end{aligned}
$$

for the linear maps induced by the inclusions $\tilde{M}_{\alpha} \subseteq \tilde{M}_{\beta}, \tilde{M}^{\beta} \subseteq \tilde{M}^{\alpha}$ and $\tilde{M}_{\alpha} \cup \tilde{M}^{\beta} \subseteq$ $\tilde{M}$.

We begin by considering the commutative diagram (61) below whose rows are the long exact sequences of the pairs $\left(\tilde{M}_{a}, \tilde{M}_{-l}\right),\left(\tilde{M}, \tilde{M}_{-l} \sqcup \tilde{M}^{l}\right),\left(\tilde{M}^{b}, \tilde{M}^{l}\right)$ for $-l<a$ and $b<l$ and vertical arrows induced by the inclusions of pairs

$$
\left(\tilde{M}_{a}, \tilde{M}_{-l}\right) \subset\left(\tilde{M}, \tilde{M}_{-l} \sqcup \tilde{M}^{l}\right) \supset\left(\tilde{M}^{b}, \tilde{M}^{l}\right) .
$$




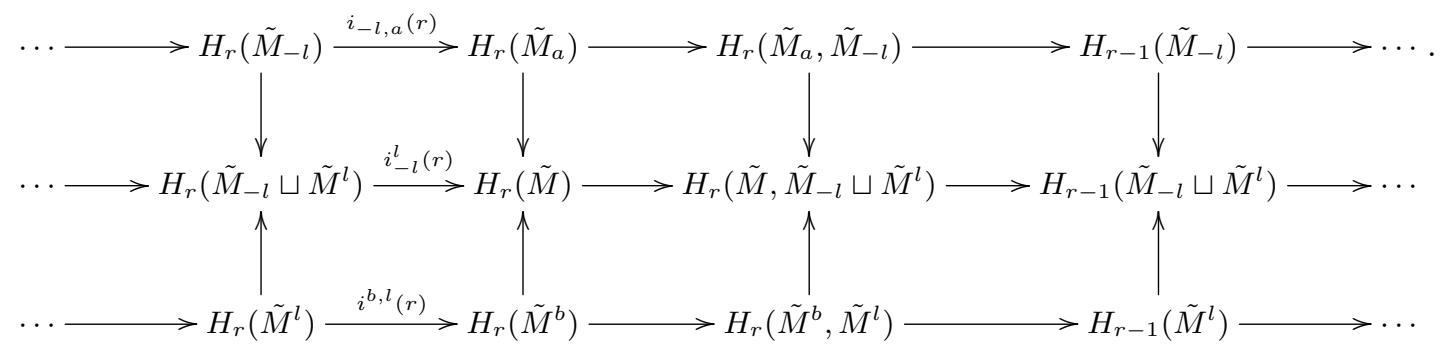

The diagram (61) leads to the following commutative diagram whose rows are short exact sequences.

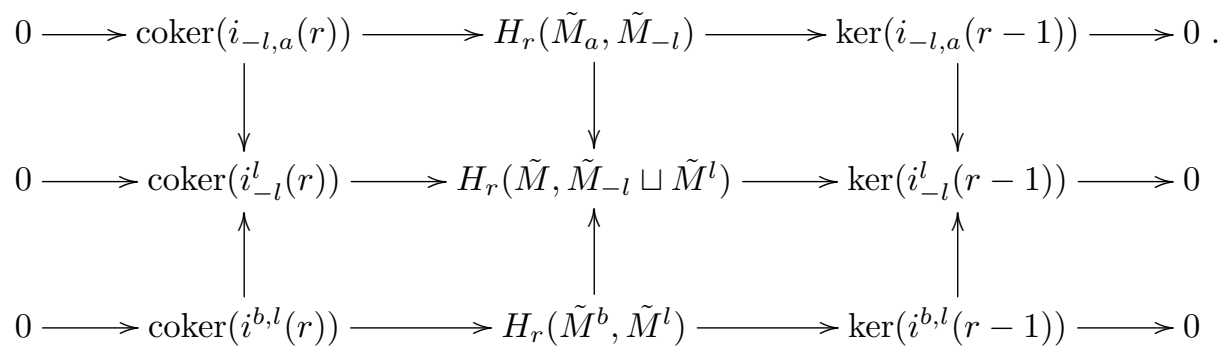

Note that there exists compatible linear maps induced by inclusions when passing from the diagram corresponding to $\left(l^{\prime}, a^{\prime}, b^{\prime}\right)$ to the diagram corresponding to $(l, a, b)$ when $l^{\prime} \geq l, a^{\prime} \geq a, b^{\prime} \leq b$. Note also that for $M$ compact and $f$ tame the set of bar codes $\mathcal{B}_{r}(f)$ is finite and therefore there is a maximal length of all bar codes say $L(f)$.

Proposition 4.2 item (b) implies on the nose that following calculations.

Proposition 6.1. Let $a, b$ fixed and suppose $l$ satisfies $a>-l, b<l$. Then

(a) $\operatorname{coker}\left(i_{-l, a}(r)\right)=\kappa\left[\mathcal{M}_{-l, a}(r)\right]$ with

$$
\begin{aligned}
\mathcal{M}_{-l, a}(r):=\left\{[\alpha, \beta] \in \mathcal{B}_{r}^{c} \mid-l<\alpha \leq a\right\} \\
\\
\cup\left\{(\alpha, \beta) \in \mathcal{B}_{r-1}^{o} \mid-l<\beta \leq a\right\} \\
\cup\left\{[\alpha, \beta) \in \mathcal{B}_{r}^{c o} \mid-l<\alpha \leq a<\beta\right\} .
\end{aligned}
$$

(b) $\operatorname{ker}\left(i_{-l, a}(r)\right)=\kappa\left[\mathcal{N}_{-l, a}(r)\right]$ with

$$
\mathcal{N}_{-l, a}(r):=\left\{[\alpha, \beta) \in \mathcal{B}_{r}^{c o} \mid \alpha \leq-l<\beta \leq a\right\} .
$$

(c) $\operatorname{coker}\left(i^{b, l}(r)\right)=\kappa\left[\mathcal{M}^{b, l}(r)\right]$ with

$$
\begin{aligned}
\mathcal{M}^{b, l}(r):=\left\{[\alpha, \beta] \in \mathcal{B}_{r}^{c} \mid b \leq \beta<l\right\} \\
\cup\left\{(\alpha, \beta) \in \mathcal{B}_{r-1}^{o} \mid b \leq \alpha<l\right\} \\
\cup\left\{(\alpha, \beta] \in \mathcal{B}_{r}^{o c} \mid \alpha<b \leq \beta<l\right\} .
\end{aligned}
$$

(d) $\operatorname{ker}\left(i^{b, l}(r)\right)=\kappa\left[\mathcal{N}^{b, l}(r)\right]$ with

$$
\mathcal{N}^{b, l}(r):=\left\{(\alpha, \beta] \in \mathcal{B}_{r}^{o c} \mid b \leq \alpha<l \leq \beta\right\} .
$$

If $2 l>L(f)$ then: 
(e) $\operatorname{coker}\left(i_{-l}^{l}(r)\right)=\kappa\left[\mathcal{M}_{-l}^{l}(r)\right]$ with

$$
\begin{aligned}
\mathcal{M}_{-l}^{l}(r):=\left\{[\alpha, \beta] \in \mathcal{B}_{r}^{c} \mid[\alpha, \beta] \subset(-l, l)\right\} \\
\\
\cup\left\{(\alpha, \beta) \in \mathcal{B}_{r-1}^{o} \mid \alpha<l, \beta>-l\right\} .
\end{aligned}
$$

(f) $\operatorname{ker}\left(i_{-l}^{l}(r)\right)=\kappa\left[\mathcal{N}_{-l}^{l}(r) \sqcup \tilde{J}_{r}(f)\right]$ with ${ }^{15}$

$$
\begin{aligned}
\mathcal{N}_{-l}^{l}(r):= & \left\{[\alpha, \beta) \in \mathcal{B}_{r}^{c o} \mid(\alpha, \beta) \ni-l\right\} \\
& \cup\left\{(\alpha, \beta] \in \mathcal{B}_{r}^{o c} \mid(\alpha, \beta) \ni l\right\} .
\end{aligned}
$$

Clearly for $l^{\prime}>l$ and $l^{\prime}-l>L(f)$ one has:

$$
\begin{aligned}
\mathcal{M}_{-l^{\prime}, a}(r) & \supseteq \mathcal{M}_{-l, a}(r), & \mathcal{N}_{-l^{\prime}, a}(r) \cap \mathcal{N}_{-l, a}(r)=\emptyset, \\
\mathcal{M}^{b, l^{\prime}}(r) & \supseteq \mathcal{M}^{b, l}(r), & \mathcal{N}^{b, l^{\prime}}(r) \cap \mathcal{N}^{b, l}(r)=\emptyset, \\
\mathcal{M}_{-l^{\prime}}^{l^{\prime}}(r) & \supseteq \mathcal{M}_{-l}^{l}(r), & \mathcal{N}_{-l^{\prime}}^{l^{\prime}}(r) \cap \mathcal{N}_{-l}^{l}(r)=\emptyset .
\end{aligned}
$$

Note that the sets $\mathcal{M}_{-}^{-}(r), \mathcal{N}_{-}^{-}(r), \tilde{\mathcal{J}}_{r}$, and $\tilde{\mathcal{J}}_{r-1}$ are all subsets of $S=\tilde{\mathcal{B}}_{r} \sqcup$ $\tilde{\mathcal{B}}_{r-1} \sqcup \tilde{\mathcal{J}}_{r} \sqcup \tilde{\mathcal{J}}_{r-1}$. Note also that all inclusions induced linear maps between the homologies involved in the diagrams (61) and (62), via the identifications of these homologies to vector spaces generated by subsets of $S$, correspond to canonical linear maps.

Recall that if $S_{1}, S_{2} \subseteq S$ then the canonical linear map $\kappa\left[S_{1}\right] \rightarrow \kappa\left[S_{2}\right]$ is the unique linear extension of the map which the identity on $S_{1} \cap S_{2}$ and zero on $S_{1} \backslash S_{2}$, cf. Definition 2.6

To finalize the verification of Step 2 for $\alpha, \beta \in \mathbb{R}$ one denotes

$$
\begin{aligned}
& \mathcal{B}_{r, \alpha}^{c}:=\tilde{\mathcal{B}}_{r,(-\infty, \alpha]}^{c}(f)=\left\{I \in \mathcal{B}_{r}^{c}(\tilde{f}) \mid I \cap(-\infty, \alpha] \neq \emptyset\right\}, \\
& \mathcal{B}_{r}^{c, \beta}:=\tilde{\mathcal{B}}_{r,[\beta, \infty)}^{c}(f)=\left\{I \in \mathcal{B}_{r}^{c}(\tilde{f}) \mid I \cap[\beta, \infty) \neq \emptyset\right\}, \\
& \mathcal{B}_{r, \alpha}^{o}:=\tilde{\mathcal{B}}_{r,(-\infty, \alpha]}^{o}(f)=\left\{I \in \mathcal{B}_{r}^{o}(\tilde{f}) \mid I \subset(-\infty, \alpha]\right\}, \\
& \mathcal{B}_{r}^{o, \beta}:=\tilde{\mathcal{B}}_{r,[\beta, \infty)}^{o}(f)=\left\{I \in \mathcal{B}_{r}^{o}(\tilde{f}) \mid I \subset[\beta, \infty)\right\} .
\end{aligned}
$$

and one considers the commutative diagram 63 below.

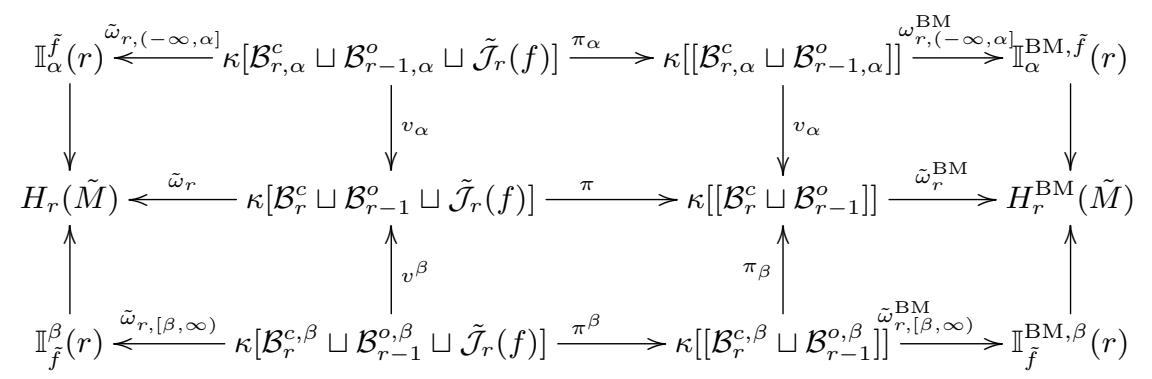

Propositions 4.2 (b) provides the left side of the diagram with $\tilde{\omega}_{r,(-\infty, \alpha]}, \tilde{\omega}_{r}$, $\tilde{\omega}_{r,[\beta, \infty)}$ isomorphisms.

By passing to the inverse limit when $l \rightarrow \infty$, Proposition 6.1 provides the right side of the diagram with $\tilde{\omega}_{r,(-\infty, \alpha]}^{\mathrm{BM}}, \tilde{\omega}_{r}^{\mathrm{BM}}$, and $\tilde{\omega}_{r,[\beta, \infty)}^{\mathrm{BM}}$ isomorphisms.

\footnotetext{
${ }^{15}$ In view of the hypothesis $(a, b)$ can not contain both $-l$ and $l$.
} 
The canonical linear maps $\pi_{\alpha}, \pi, \pi^{\beta}, v_{\alpha}$ and $v^{\beta}$ defined by the sets in brackets when regarded as subsets in $\tilde{\mathcal{B}}_{r}(f) \sqcup \tilde{\mathcal{B}}_{r-1}(f) \sqcup \tilde{\mathcal{J}}_{r}(f) \sqcup \tilde{\mathcal{J}}_{r-1}(f)$ (cf. Definition 2.6 provide the middle of the diagram.

Diagram 63 implies that $\mathbb{I}_{\alpha}^{\tilde{f}}(r) \cap \mathbb{I}_{\tilde{f}}^{\beta}(r)$ identifies to

$$
\kappa\left[\left(\tilde{\mathcal{B}}_{r, \alpha}^{c} \cap \tilde{\mathcal{B}}_{r}^{c, \beta}\right) \sqcup\left(\tilde{\mathcal{B}}_{r-1, \alpha}^{o} \cap \tilde{\mathcal{B}}_{r-1}^{o, \beta}\right) \sqcup \tilde{\mathcal{J}}_{r}(f)\right]
$$

and $\mathbb{I}_{\alpha}^{\mathrm{BM}, \tilde{f}}(r) \cap \mathbb{I}_{\tilde{f}}^{\mathrm{BM}, \beta}(r)$ identifies to

$$
\kappa\left[\left[\left(\tilde{\mathcal{B}}_{r, \alpha}^{c} \cap \tilde{\mathcal{B}}_{r}^{c, \beta}\right) \sqcup\left(\tilde{\mathcal{B}}_{r-1, \alpha}^{o} \cap \tilde{\mathcal{B}}_{r-1}^{o, \beta}\right)\right]\right] .
$$

which in view of the fact that the set in brackets is finite (hence there is no difference between $\kappa[[\cdots]]$ and $\kappa[\cdots])$ imply the exactness of the sequence

$$
0 \rightarrow \kappa\left[\tilde{\mathcal{J}}_{r}(f)\right] \rightarrow \mathbb{I}_{\alpha}^{\tilde{f}}(r) \cap \mathbb{I}_{\tilde{f}}^{\beta}(r) \rightarrow \mathbb{I}_{\alpha}^{\mathrm{BM}, \tilde{f}}(r) \cap \mathbb{I}_{\tilde{f}}^{\mathrm{BM}, \beta}(r) \rightarrow 0 .
$$

Note that $\mathbb{I}_{\alpha}^{\tilde{f}}(r) \cap \mathbb{I}_{\tilde{f}}^{\beta}(r) \rightarrow \mathbb{I}_{\alpha}^{\mathrm{BM}, \tilde{f}}(r) \cap \mathbb{I}_{\tilde{f}}^{\mathrm{BM}, \beta}(r)$ is the composition $\tilde{\omega}_{r}^{\mathrm{BM}} \cdot \pi \cdot \tilde{\omega}_{r}^{-1}$. The exact sequence above implies

$$
F_{r}^{B M, \tilde{f}}(a, b)+\sharp \tilde{\mathcal{J}}_{r}(f)=F_{r}^{\tilde{f}}(a, b)
$$

which finalizes Step 2.

As a side remark note that by passing to the inverse limit when $l \rightarrow \infty$ Proposition 6.1 and Diagram 61) led to the following calculations used in the next section.

\section{Proposition 6.2.}

(1) $H_{r}^{\mathrm{BM}}(\tilde{X})=\kappa\left[\left[\tilde{S}_{r}(f) \sqcup \tilde{\mathcal{J}}_{r-1}(f)\right]\right]=\kappa\left[\left[\tilde{\mathcal{B}}_{r}^{c}(f) \sqcup \tilde{\mathcal{B}}_{r-1}^{o}(f) \sqcup \tilde{\mathcal{J}}_{r-1}(f)\right]\right]$.

(2) $\mathbb{I}_{a}^{\mathrm{BM}, \tilde{f}}(r)=\kappa\left[\left[\tilde{S}_{r,(-\infty, a]}(f)\right]\right]=\kappa\left[\left[\tilde{\mathcal{B}}_{r,(-\infty, a]}^{c}(f) \sqcup \tilde{\mathcal{B}}_{r-1,(-\infty, a]}^{o}(f)\right]\right]$.

(3) $H_{r}^{\mathrm{BM}}\left(\tilde{X}_{a}\right)=\kappa\left[\left[\tilde{S}_{r,(-\infty, a]}^{\prime}(f)\right]\right]=\kappa\left[\left[\tilde{\mathcal{B}}_{r,(-\infty, a]}^{c o} \sqcup \tilde{S}_{r,(-\infty, a]}(f)\right]\right]$.

(4) $\mathbb{I}_{\tilde{f}}^{\mathrm{BM}, b}(r)=\kappa\left[\left[\tilde{S}_{r,[b, \infty)}(f)\right]\right]=\kappa\left[\left[\tilde{\mathcal{B}}_{r,([b, \infty)}^{c}(f) \sqcup \tilde{\mathcal{B}}_{r-1,[b, \infty)}^{o}(f)\right]\right]$.

(5) $H_{r}^{\mathrm{BM}}\left(\tilde{X}^{b}\right)=\kappa\left[\left[\tilde{S}_{r,(b, \infty)}^{\prime}(f)\right]\right]=\kappa\left[\left[\tilde{\mathcal{B}}_{r,[b, \infty)}^{o c} \sqcup \tilde{S}_{r,(-\infty, a]}(f)\right]\right]$.

The canonical linear map $H_{r}(\tilde{X}) \rightarrow H_{r}^{B M(}(\tilde{X})$ can be also read off from Diagram 63 in terms of barcodes. It is exactly the unique linear map which is identity on $\mathcal{B}_{r}^{c} \sqcup \mathcal{B}_{r-1}^{o}$ and zero on $\widetilde{\mathcal{J}}_{r}(f)$.

\section{STEP 3:}

In view of the fact that by $\delta_{r}^{\tilde{f}}(a, b)=\mu_{r}^{\tilde{f}}((a-\epsilon, a+\epsilon] \times[b-\epsilon, b+\epsilon)$ for $\epsilon$ small enough, cf. (38), and of Proposition (5.4) the equality in Step 3 follows.

\section{The mixed Bar codes. Proof of Theorem 1.5}

Let $f: X \rightarrow \mathbb{S}^{1}$ be a tame angle-valued map and $\tilde{f}: \tilde{X} \rightarrow \mathbb{R}$ be the infinite cyclic covering of $f$.The sets $\mathcal{B}_{r}^{c o}(f)$ and $\mathcal{B}_{r}^{o c}(f)$ which define the configuration $C_{r}^{m}(f)$, cf. Section 3, are equivalence classes modulo translation by integer multiple of $2 \pi$ of the bar codes of $\tilde{\mathcal{B}}_{r}^{c o}(f)=\mathcal{B}_{r}^{c o}(\tilde{f})$ and $\tilde{\mathcal{B}}_{r}^{o c}(f)=\mathcal{B}_{r}^{o c}(\tilde{f})$.

Note also that an interval $[a, b)$ is an element in $\mathcal{B}_{r}^{c o}(\tilde{f})$ with multiplicity $k$ iff $(a, b)$ is an $r$-persistence bar code in the sense of [17] with the same multiplicity for the sublevel filtration associated with $\tilde{f}$. Similarly the interval $(a, b]$ is an element in $\mathcal{B}_{r}^{o c}(\tilde{f})$ with multiplicity $k$ if $(-b,-a)$ is an $r$-persistence bar code with the same multiplicity for the sublevel-filtration associated with $-\tilde{f}$. Recall that a point 
TOPOLOGY OF ANGLE VALUED MAPS, BAR CODES AND JORDAN BLOCKS.

$\langle a, b\rangle \in\left(\mathbb{R}^{2} \backslash \Delta\right) / \mathbb{Z}$ is in the support of $C_{r}^{m}(f)$ with multiplicity $k$ iff $[a, b) \in \mathcal{B}_{r}^{\text {co }}(f)$ with multiplicity $k$ when $a>b$, or $(a, b] \in \mathcal{B}_{r}^{o c}(f)$ when $a<b$ with multiplicity $k$.

The stability result in [11], the MAIN THEOREM, remains valid with the same proof if instead of real-valued tame maps one considers angle-valued tame maps $f: X \rightarrow \mathbb{S}^{1}$.

More precisely the assignment $f \rightsquigarrow \mathcal{B}_{r}^{c o}(f)$, with $\mathcal{B}_{r}^{c o}(f)$ viewed as a multiset (= configuration of points) in $\mathbb{R}_{+}^{2} / \mathbb{Z} \subset\left(\mathbb{R}^{2} \backslash \Delta\right) / \mathbb{Z}=\mathbb{T} \backslash \Delta_{\mathbb{T}}$ is continuous w.r. to the compact open topology on $C_{\xi, t}\left(X, \mathbb{S}^{1}\right)$ and the topology induced by the bottleneck metric on the space of configurations $\operatorname{Confg}\left(\left(\mathbb{R}_{+}^{2} / \mathbb{Z}\right) \subset \operatorname{Confg}\left(\left(\mathbb{R}^{2} \backslash \Delta\right) / \mathbb{Z}\right)\right.$.

Similarly the assignment $f \rightsquigarrow \mathcal{B}_{r}^{o c}(f)$, with $\mathcal{B}_{r}^{o c}(f)$ viewed as a multi set in $\mathbb{R}_{-}^{2} / \mathbb{Z} \subset\left(\mathbb{R}^{2} \backslash \Delta\right) / \mathbb{Z}$, is continuous w.r. to the same topologies. The spaces $\mathbb{R}_{+}^{2}:=$ $\{(x, y) \mid x<y\}$ and $\mathbb{R}_{-}^{2}:=\{(x, y) \mid x<y\}$ are invariant w.r. to the action $\mathbb{Z} \times \mathbb{R}^{2} \rightarrow \mathbb{R}^{2}$ given by $(n,(x, y)) \rightarrow(x+2 n \pi, y+2 n \pi)$.

The bottleneck metric defined in [11] induces the bottleneck topology defined without metric in Section 1 . In terms of the configuration $C_{r}^{m}(f)$ the stability result in [11] can be reformulated in the following way.

Theorem The assignment $f \mapsto C_{r}^{m}(f)$ from $C_{\xi, t}\left(X, \mathbb{S}^{1}\right)$ the space of tame maps equipped with the compact open topology to $\operatorname{Confg}(\mathbb{T} \backslash \Delta)$ equipped with the bottleneck topology for case $(X, K)=\left(\mathbb{T}, \Delta_{\mathbb{T}}\right)$ as described in Section 1 is continuous. ${ }^{16}$

This because the bottleneck metric induces the bottleneck topology. This Theorem is exactly Theorem 1.5 .

In parallelism with the configuration $C_{r}(f)$, for the proof of Theorem 1.6 one can identify the configuration $C_{r}^{m}(f)$ with the map $\delta_{r}^{m, f}: \mathbb{T} \backslash \Delta_{\mathbb{T}} \rightarrow \mathbb{Z}_{\geq 0}$. This map can be derived from the function of two variables $T_{r}^{\tilde{f}}: \mathbb{R}^{2} \backslash \Delta \rightarrow \mathbb{Z}_{\geq 0}$ in a similar manner to the description of the configuration $C_{r}(f)$ in Section 5 The function $T_{r}^{\tilde{f}}$ is defined by:

$$
T_{r}^{\tilde{f}}(a, b):= \begin{cases}\operatorname{dim} \operatorname{ker}\left(H_{r}\left(\tilde{X}_{a}\right) \rightarrow H_{r}\left(\tilde{X}_{b}\right)\right) & \text { if } a<b \\ \operatorname{dim} \operatorname{ker}\left(H_{r}\left(\tilde{X}^{a}\right) \rightarrow H_{r}\left(\tilde{X}^{b}\right)\right) & \text { if } a>b .\end{cases}
$$

If $f$ is tame then so is $\tilde{f}$ and the limit

$$
\begin{aligned}
\delta_{r}^{m, \tilde{f}}(a, b)=\lim _{\epsilon \rightarrow 0}\left(T_{r}^{\tilde{f}}(a+\epsilon, b+\epsilon)-\right. & T_{r}^{\tilde{f}}(a-\epsilon, b+\epsilon) \\
& \left.+T_{r}^{\tilde{f}}(a-\epsilon, b-\epsilon)-T_{r}^{\tilde{f}}(a+\epsilon, b-\epsilon)\right)
\end{aligned}
$$

exists and defines the function $\delta_{r}^{m, \tilde{f}}$ which satisfies

$$
\delta_{r}^{m, \tilde{f}}(a, b)=\delta_{r}^{m, \tilde{f}}(a+2 \pi, b+2 \pi) .
$$

Then, as in Section 5 , one defines the function $\delta_{r}^{m, f}: \mathbb{T} \backslash \Delta_{T} \rightarrow \mathbb{Z}_{\geq 0}$ by

$$
\delta_{r}^{m, f}(\langle a, b\rangle):=\delta_{r}^{m, \tilde{f}}(a, b) .
$$

As in Section 5 Proposition 5.7, using Proposition 4.2 one verifies that $\delta_{r}^{m, f}$ and $C_{r}^{m}(f)$ are equal.

${ }^{16}$ A direct verification of Theorem 1.5 without reference to [17] can be found in the forthcoming book 8 . 
Proposition 7.1. If $f$ is a tame real- or angle-valued map defined on $X$, a compact $A N R$, then $\delta_{r}^{m, f}$ and $C_{r}^{m}(f)$ are equal $\mathbb{Z}_{\geq 0}$-valued functions defined on $\mathbb{R}^{2} \backslash \Delta$ or $\mathbb{T} \backslash \Delta_{\mathbb{T}}$.

The proof is exactly the same as of Proposition. 5.7 provided one replaces (47) and $(48)$ by (66) which follows in a straightforward manner from Proposition 4.2 precisely the following calculations.

$$
\begin{gathered}
T_{r}^{f}\left(c_{i}, c_{j}\right)=\sharp\left\{I \in \mathcal{B}_{r}^{c, o} \mid I=\left[c_{i}, c_{j}\right)\right\} \text { if } \mathrm{c}_{\mathrm{i}}<\mathrm{c}_{\mathrm{j}} \\
T_{r}^{f}\left(c_{i}, c_{j}\right)=\sharp\left\{I \in \mathcal{B}_{r}^{c, o} \mid I=\left[c_{i}, c_{j}\right)\right\} \text { if } \mathrm{c}_{\mathrm{i}}>\mathrm{c}_{\mathrm{j}} .
\end{gathered}
$$

In order to prove Theorem 1.6 we first recall some notations.

For $\tilde{f}: \tilde{X} \rightarrow \mathbb{R}$ the infinite cyclic covering of the tame map $f: X \rightarrow \mathbb{S}^{1}, a, b \in \mathbb{R}, a \leq$ $b$

- one considers the inclusion induced linear maps :

$$
\begin{aligned}
i_{a}(r): H_{r}\left(\tilde{X}_{a}\right) & \rightarrow H_{r}(\tilde{X}), & i_{a}^{\mathrm{BM}}(r): H_{r}^{\mathrm{BM}}\left(\tilde{X}_{a}\right) & \rightarrow H_{r}^{\mathrm{BM}}(\tilde{X}), \\
i^{a}(r): H_{r}\left(\tilde{X}^{a}\right) & \rightarrow H_{r}(\tilde{X}), & i^{\mathrm{BM}, a}(r): H_{r}^{\mathrm{BM}}\left(\tilde{X}^{a}\right) & \rightarrow H_{r}^{\mathrm{BM}}(\tilde{X}) . \\
i_{a, b}(r): H_{r}\left(\tilde{X}_{a}\right) & \rightarrow H_{r}\left(\tilde{X}_{b}\right), & i_{a, b}^{\mathrm{BM}}(r): H_{r}^{\mathrm{BM}}\left(\tilde{X}_{a}\right) & \rightarrow H_{r}^{\mathrm{BM}}\left(\tilde{X}_{b}\right), \\
i^{b, a}(r): H_{r}\left(\tilde{X}^{b}\right) & \rightarrow H_{r}\left(\tilde{X}^{a}\right), & i^{\mathrm{BM}, b, a}(r): H_{r}^{\mathrm{BM}}\left(\tilde{X}^{b}\right) & \rightarrow H_{r}^{\mathrm{BM}}\left(\tilde{X}^{a}\right) .
\end{aligned}
$$

- one defines

$$
\begin{aligned}
\mathbb{K}_{a}(r) & :=\operatorname{ker} i_{a}(r), & \mathbb{K}_{a}^{\mathrm{BM}}(r) & :=\operatorname{ker} i_{a}^{\mathrm{BM}}(r), \\
\mathbb{K}^{a}(r) & :=\operatorname{ker} i^{a}(r), & \mathbb{K}^{\mathrm{BM}, a}(r) & :=\operatorname{ker} i^{\mathrm{BM}, a}(r),
\end{aligned}
$$

and

- one denotes by

$$
\begin{aligned}
\tilde{\iota}_{a, b}(r): & \mathbb{K}_{a}(r) \rightarrow \mathbb{K}_{b}(r), & \tilde{\iota}_{a, b}^{\mathrm{BM}}(r): \mathbb{K}_{a}^{\mathrm{BM}}(r) & \rightarrow \mathbb{K}_{b}^{B M}(r), \\
\tilde{\iota}^{b, a}(r): \mathbb{K}^{b}(r) & \rightarrow \mathbb{K}^{a}(r), & \tau^{\mathrm{BM}, b, a}(r): \mathbb{K}^{\mathrm{BM}, b}(r) & \rightarrow \mathbb{K}^{B M, a}(r)
\end{aligned}
$$

the restrictions of $i_{a, b}(r), i_{a, b}^{\mathrm{BM}}(r)$ and $i^{b, a}(r), i^{\mathrm{BM}, b, a}(r)$ to these kernels.

Note that in view of the definition 64

$$
\begin{aligned}
& T_{r}^{f}(a, b)=\operatorname{ker} i a, b(r), a<b \\
& T_{r}^{f}(a, b)=\operatorname{ker} i^{a, b}(r), a>b
\end{aligned}
$$

by elementary linear algebra argument

$$
\begin{aligned}
& \operatorname{ker} \tilde{\iota}_{a, b}(r)=\operatorname{ker} i_{a, b}(r) \\
& \operatorname{ker} \tilde{\iota}^{a, b}(r)=\operatorname{ker} i^{a, b}(r)
\end{aligned}
$$

and In view of the calculations of the Borel-Moore homology of $\tilde{X}^{a}, \tilde{X}_{a}, \tilde{X} \mathrm{cf}$ Proposition 6.2 and the description of the linear maps from homology to BorelMoore homology one concludes that

$$
\begin{aligned}
\mathbb{K}(r) & =\mathbb{K}^{\mathrm{BM}}(r) \\
\tilde{\iota}(r) & =\tilde{\iota}^{\mathrm{BM}}(r)
\end{aligned}
$$

Proposition 4.2 permits to describe the vector spaces $\mathbb{K}_{a}(r), \mathbb{K}^{a}(r), \operatorname{ker}\left(\tilde{\iota}_{a, b}(r)\right)$, $\operatorname{coker}\left(\tilde{\iota}_{a, b}(r)\right), \operatorname{ker}\left(\tilde{\iota}^{b, a}(r)\right), \operatorname{coker}\left(\tilde{\iota}^{b, a}(r)\right)$ in terms of mixed bar codes as in Proposition 7.2 below. The verification is a straightforward reading of Proposition 4.2 
Proposition 7.2. Suppose $f: X \rightarrow \mathbb{S}^{1}$ is a tame map with $\tilde{f}: \tilde{X} \rightarrow \mathbb{R}$ its infinite cyclic covering, and $a, b$ real numbers with $a \leq b$. Then:

$$
\begin{aligned}
\mathbb{K}_{a}(r) & =\kappa\left[\left\{I \in \tilde{\mathcal{B}}_{r}^{c o}(f) \mid I \ni a\right\}\right], \\
\mathbb{K}^{a}(r) & =\kappa\left[\left\{I \in \tilde{\mathcal{B}}_{r}^{o c}(f) \mid I \ni a\right\}\right], \\
\operatorname{ker}\left(\tilde{\iota}_{a, b}(r)\right) & =\kappa\left[\left\{I \in \tilde{\mathcal{B}}_{r}^{c o}(f) \mid I \ni a, b \notin I\right\}\right], \\
\operatorname{coker}\left(\tilde{\iota}_{a, b}(r)\right) & =\kappa\left[\left\{I \in \tilde{\mathcal{B}}_{r}^{c o}(f) \mid I \ni b, a \notin I\right\}\right], \\
\operatorname{ker}\left(\tilde{\iota}^{b, a}(r)\right) & =\kappa\left[\left\{I \in \tilde{\mathcal{B}}_{r}^{o c}(f) \mid I \ni b, a \notin I\right\}\right], \\
\operatorname{coker}\left(\tilde{\iota}^{b, a}(r)\right) & =\kappa\left[\left\{I \in \tilde{\mathcal{B}}_{r}^{o c}(f) \mid I \ni a, b \notin I\right\}\right] .
\end{aligned}
$$

Note that $\mathbb{K}_{a}(r)$ and $\mathbb{K}^{a}(r)$ are finite dimensional vector spaces.

In view of the tameness of $f$ and of $(65),(67)$ and $(68)$ one concludes that for $a<b$ and $\epsilon$ small enough

$$
\begin{array}{r}
\delta_{k}^{m, \tilde{f}}(a, b)=\operatorname{dim} \operatorname{ker}\left(i_{a+\epsilon, b+\epsilon}(k)\right)-\operatorname{dim} \operatorname{ker}\left(i_{a-\epsilon, b+\epsilon}(k)\right) \\
-\operatorname{dim} \operatorname{ker}\left(i_{a+\epsilon, b-\epsilon}(k)\right)+\operatorname{dim} \operatorname{ker}\left(i_{a-\epsilon, b-\epsilon}(k)\right) \\
=\operatorname{dim} \operatorname{ker}\left(\tilde{\iota}_{a+\epsilon, b+\epsilon}(k)\right)-\operatorname{dim} \operatorname{ker}\left(\tilde{\iota}_{a-\epsilon, b+\epsilon}(k)\right) \\
\quad-\operatorname{dim} \operatorname{ker}\left(\tilde{\iota}_{a+\epsilon, b-\epsilon}(k)\right)+\operatorname{dim} \operatorname{ker}\left(\tilde{\iota}_{a-\epsilon, b-\epsilon}(k)\right)
\end{array}
$$

and for $a>b$ and $\epsilon$ small enough one has

$$
\begin{aligned}
\delta_{k}^{m, \tilde{f}}(a, b)= & \operatorname{dim} \operatorname{ker}\left(i^{a-\epsilon, b-\epsilon}(r)\right)-\operatorname{dim} \operatorname{ker}\left(i^{a+\epsilon, b-\epsilon}(r)\right) \\
& -\operatorname{dim} \operatorname{ker}\left(i^{a-\epsilon, b+\epsilon}(r)\right)+\operatorname{dim} \operatorname{ker}\left(i^{a+\epsilon, b+\epsilon}(r)\right) \\
= & \operatorname{dim} \operatorname{ker}\left(\tilde{\iota}^{a-\epsilon, b-\epsilon}(r)\right)-\operatorname{dim} \operatorname{ker}\left(\tilde{\iota}^{a+\epsilon, b-\epsilon}(r)\right) \\
& -\operatorname{dim} \operatorname{ker}\left(\tilde{\iota}^{a-\epsilon, b+\epsilon}(r)\right)+\operatorname{dim} \operatorname{ker}\left(\tilde{\iota}^{a+\epsilon, b+\epsilon}(r)\right) .
\end{aligned}
$$

Next observe that the long exact sequence for the pair $\left(\tilde{X}, \tilde{X}_{\alpha}\right), \alpha \in \mathbb{R}$

$$
\begin{aligned}
\cdots \rightarrow H_{n-r}(\tilde{X}) \stackrel{j^{\alpha}(n-r)}{\longrightarrow} H_{n-r}\left(\tilde{X}, \tilde{X}^{\alpha}\right) \stackrel{\partial^{\alpha}(n-r)}{\longrightarrow} & \\
& \rightarrow H_{n-r-1}\left(\tilde{X}^{\alpha}\right) \stackrel{i^{\alpha}(n-r-1)}{\longrightarrow} H_{n-1-r}(\tilde{X}) \rightarrow \cdots
\end{aligned}
$$

provides the isomorphism

$$
\hat{\partial}^{\alpha}(n-r): \operatorname{coker}\left(j^{\alpha}(n-r)\right) \rightarrow \operatorname{ker}\left(i^{\alpha}(n-r-1)\right)=\mathbb{K}^{\alpha}(n-r-1)
$$

which, being "natural" w.r. to the inclusion of pairs $\left(\tilde{X}, \tilde{X}^{\beta}\right) \subseteq\left(\tilde{X}, \tilde{X}^{\alpha}\right)$ for $\alpha \leq \beta$, makes the diagram below commutative.

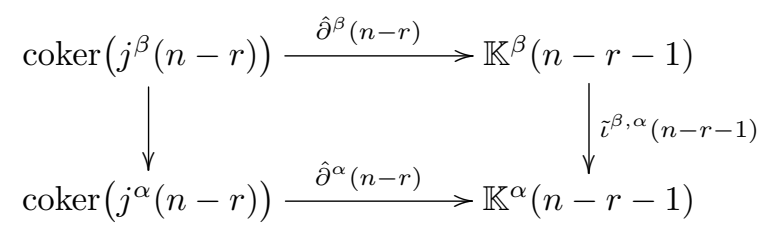

\section{Proof of Theorem 1.6}

Suppose now that $X=M$ is a closed $\kappa$-orientable $n$-dimensional manifold and $\alpha$ is a regular value of $\tilde{f}$. Poincaré duality for the manifold $\tilde{M}$ and for the pairs 
$\left(\tilde{M}, \tilde{M}_{\alpha}\right)$ and $\left(\tilde{M}, \tilde{M}^{\alpha}\right)$ provides the commutative diagram

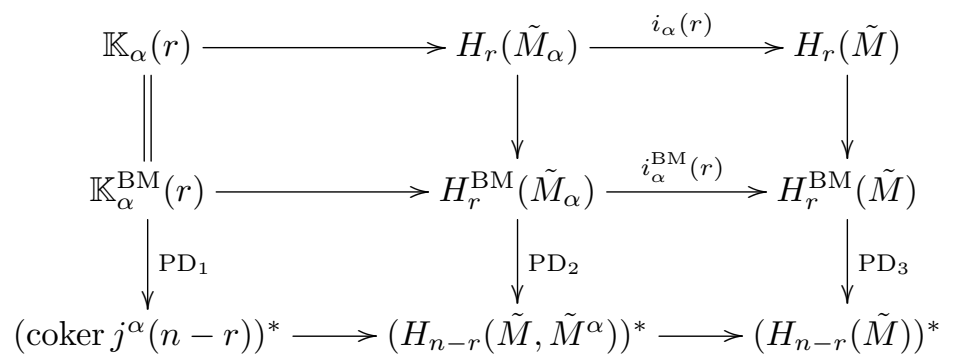

with the bottom vertical arrows $P D_{1}, P D_{2}, P D_{3}$ isomorphisms. This is because $P D_{2}$ and $P D_{3}$ which appear in (53) are isomorphisms as indicated in Section 5 The diagram is natural w.r. to the inclusion of pairs $\left(X, X_{\alpha}\right) \subseteq\left(X, X_{\beta}\right)$, provided $\alpha$ and $\beta$ are regular values. It leads to the following commutative diagram whose vertical arrows are all isomorphisms:

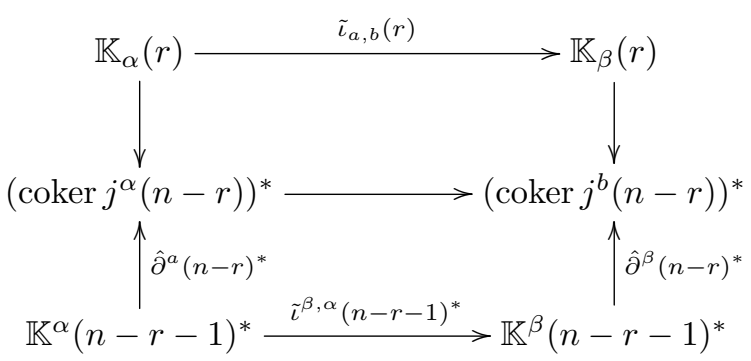

To finalize the proof of Theorem 1.6. recall that the tameness of $f$ implies the tameness of $\tilde{f}$ and for $a, b$ critical values and $\epsilon<\epsilon(f)$, the numbers $a \pm \epsilon, b \pm \epsilon$ are regular values, therefore by 75 one has

$$
\tilde{\iota}_{a \pm \epsilon, b \pm \epsilon^{\prime}}(r)=\left(\tilde{\iota}^{b \pm \epsilon, a \pm \epsilon^{\prime}}(n-1-r)\right)^{*} .
$$

The equations (70), 71], and (76) imply $\delta_{r}^{m, \tilde{f}}(a, b)=\delta_{n-r-1}^{m, \tilde{f}}(b, a)$ and then $C_{r}^{m}(f)(\langle a, b\rangle)=\delta_{r}^{m, f}(\langle a, b\rangle)=\delta_{n-r-1}^{m, f}(\langle b, a\rangle)=C_{n-r-1}^{m}(f)(\langle b, a\rangle)$. equality which establishes Theorem 1.6.

\section{Linear relations and monodromy. Proof of Theorem 1.4}

We begin this section with a discussion of linear relations. To every linear relation $R: V \rightsquigarrow V$ we associate a linear relation $R_{\text {reg }}: V_{\text {reg }} \rightsquigarrow V_{\text {reg }}$ on a subquotient, $V_{\text {reg}}$, of $V$. In Proposition 8.2 we show that $R_{\text {reg }}$ is a linear isomorphism. If $V$ is a finite dimensional vector space, then, according to the Krull-Remak-Schmidt theorem, $R$ can be decomposed as a direct sum of indecomposable linear relations, $R \cong R_{1} \oplus \cdots \oplus R_{N}$, where the factors $R_{i}: V_{i} \rightsquigarrow V_{i}$ are unique up to permutation and isomorphism. In this case, $R_{\text {reg }}$ is isomorphic to the direct sum of factors which are indecomposable linear isomorphisms see Proposition 8.3. For linear relations on complex vector spaces $R_{\text {reg }}$ can easily be derived using the detailed structure theorem in [33. Here we will be concerned with vector spaces over arbitrary fields. Most of this material can be developed for linear relations on modules over commutative rings, and this is the setting for the basic definitions, although in this paper we are interested only in the case of vector spaces. 
In the second part of this section, we consider the level $X_{\theta}=f^{-1}(\theta)$ associated with a continuous map $f: X \rightarrow S^{1}$ and a value $\theta \in S^{1}$ s.t. $f^{-1}(\theta)$ is an ANR. Using the corresponding infinite cyclic covering $\tilde{X} \rightarrow X$ one obtains a linear relation $R_{\theta}$ on $H_{*}\left(X_{\theta}\right)$, see Section 1 or 81 below. We will show that $\left(R_{\theta}\right)_{\text {reg }}$ is conjugate to the isomorphism induced by the fundamental deck transformation on

$$
\operatorname{ker}\left(H_{*}(\tilde{X}) \rightarrow H_{*}^{\mathrm{Nov},-}(\tilde{X}) \oplus H_{*}^{\mathrm{Nov},+}(\tilde{X})\right)
$$

see Theorem 8.14. Here $H_{*}^{\text {Nov, }} \pm(\tilde{X})$ denote Novikov type homology groups explained below. The second part of Theorem 8.14 implies that this is isomorphic to $\operatorname{ker}\left(H_{r}(\tilde{X}) \rightarrow H_{r}^{N}\left(X, \xi_{f}\right)\right)$ considered in Section 4

This result holds true without compactness assumptions on $X$ (and with arbitrary coefficients not necessary in a field). It implies that $R_{\text {reg }}$ is a homotopy invariant of $f$.

At the end of Section 8.2 we will give a proof of Theorem 1.4

8.1. Linear relations. Suppose $V$ and $W$ are two modules over a fixed commutative ring. Recall that a linear relation from $V$ to $W$ can be considered as a submodule $R \subseteq V \times W$. Notationally, we indicate this situation by $R: V \rightsquigarrow W$. For $v \in V$ and $w \in W$ we write $v R w$ iff $v$ is in relation with $w$, i.e. $(v, w) \in R$. Every module homomorphism $V \rightarrow W$ can be regarded as a linear relation $V \rightsquigarrow W$ in a natural way. If $U$ is another module, and $S: W \rightsquigarrow U$ is a linear relation, then the composition $S R: V \rightsquigarrow U$ is the linear relation defined by $v(S R) u$ iff there exists $w \in W$ such that $v R w$ and $w S u$. Clearly, this is an associative composition generalizing the ordinary composition of module homomorphisms. For the identical relations we have $R \operatorname{Id}_{V}=R$ and $\operatorname{Id}_{W} R=R$. Modules over a fixed commutative ring and linear relations thus constitute a category. If $R: V \rightsquigarrow W$ is a linear relation we define a linear relation $R^{\dagger}: W \rightsquigarrow V$ by $w R^{\dagger} v$ iff $v R w$. Clearly, $R^{\dagger \dagger}=R$ and $(S R)^{\dagger}=R^{\dagger} S^{\dagger}$.

A linear relation $R: V \rightsquigarrow W$ gives rise to the following submodules:

$$
\begin{aligned}
\operatorname{dom}(R) & :=\{v \in V \mid \exists w \in W: v R w\} \\
\operatorname{img}(R) & :=\{w \in W \mid \exists v \in V: v R w\} \\
\operatorname{ker}(R) & :=\{v \in V \mid v R 0\} \\
\operatorname{mul}(R) & :=\{w \in W \mid 0 R w\}
\end{aligned}
$$

Clearly, $\operatorname{ker}(R) \subseteq \operatorname{dom}(R) \subseteq V$, and $W \supseteq \operatorname{img}(R) \supseteq \operatorname{mul}(R)$. Note that $R$ is a homomorphism (map) iff $\operatorname{dom}(R)=V$ and $\operatorname{mul}(R)=0$. One readily verifies:

Lemma 8.1. For a linear relation $R: V \rightsquigarrow W$ the following are equivalent:

(a) $R$ is an isomorphism in the category of modules and linear relations.

(b) $\operatorname{dom}(R)=V, \operatorname{img}(R)=W, \operatorname{ker}(R)=0$, and $\operatorname{mul}(R)=0$.

(c) $R$ is an isomorphism of modules.

In this case $R^{-1}=R^{\dagger}$. 
For a linear relation $R: V \rightsquigarrow V$, we introduce the following submodules:

$$
\begin{aligned}
K_{+} & :=\left\{v \in V \mid \exists k \exists v_{i} \in V: v R v_{1} R v_{2} R \cdots R v_{k} R 0\right\} \\
K_{-} & :=\left\{v \in V \mid \exists k \exists v_{i} \in V: 0 R v_{-k} R \cdots R v_{-2} R v_{-1} R v\right\} \\
D_{+} & :=\left\{v \in V \mid \exists v_{i} \in V: v R v_{1} R v_{2} R v_{3} R \cdots\right\} \\
D_{-} & :=\left\{v \in V \mid \exists v_{i} \in V: \cdots R v_{-3} R v_{-2} R v_{-1} R v\right\} \\
D:=D_{-} \cap D_{+} & =\left\{v \in V \mid \exists v_{i} \in V: \cdots R v_{-2} R v_{-1} R v R v_{1} R v_{2} R \cdots\right\},
\end{aligned}
$$

Clearly, $K_{-} \subseteq D_{-} \subseteq V \supseteq D_{+} \supseteq K_{+}$. Also note that passing from $R$ to $R^{\dagger}$, the roles of + and - get interchanged. Moreover, we introduce a linear relation on the quotient module

$$
V_{\text {reg }}:=\frac{D}{\left(K_{-}+K_{+}\right) \cap D}
$$

defined as the composition

$$
V_{\text {reg }}=\frac{D}{\left(K_{-}+K_{+}\right) \cap D} \stackrel{\pi^{\dagger}}{\rightsquigarrow} D \stackrel{\iota}{\rightsquigarrow} V \stackrel{R}{\rightsquigarrow} V \stackrel{\iota^{\dagger}}{\rightsquigarrow} D \stackrel{\pi}{\rightsquigarrow} \frac{D}{\left(K_{-}+K_{+}\right) \cap D}=V_{\text {reg }},
$$

where $\iota$ and $\pi$ denote the canonical inclusion and projection, respectively. In other words, two elements in $V_{\text {reg }}$ are related by $R_{\text {reg }}$ iff they admit representatives in $D$ which are related by $R$. We refer to $R_{\text {reg }}$ as the regular part of $R$.

Proposition 8.2. The relation $R_{\text {reg }}: V_{\text {reg }} \rightsquigarrow V_{\text {reg }}$ is an isomorphism of modules. Moreover, the natural inclusion induces a canonical isomorphism

$$
V_{\text {reg }}=\frac{D}{\left(K_{-}+K_{+}\right) \cap D} \stackrel{\cong}{\rightrightarrows} \frac{\left(K_{-}+D_{+}\right) \cap\left(D_{-}+K_{+}\right)}{K_{-}+K_{+}}
$$

which intertwines $R_{\text {reg }}$ with the relation induced on the right hand side quotient.

Proof. Clearly, (78) is well defined and injective. To see that it is onto let

$$
x=k_{-}+d_{+}=d_{-}+k_{+} \in\left(K_{-}+D_{+}\right) \cap\left(D_{-}+K_{+}\right),
$$

where $k_{ \pm} \in K_{ \pm}$and $d_{ \pm} \in D_{ \pm}$. Thus

$$
x-k_{-}-k_{+}=d_{+}-k_{+}=d_{-}-k_{-} \in D_{+} \cap D_{-}=D .
$$

We conclude $x \in D+K_{-}+K_{+}$, whence $(78)$ is onto. We will next show that this isomorphism intertwines $R_{\text {reg }}$ with the relation induced on the right hand side. To do so, suppose $x R \tilde{x}$ where

$$
\begin{aligned}
& x=k_{-}+d_{+}=d_{-}+k_{+} \in\left(K_{-}+D_{+}\right) \cap\left(D_{-}+K_{+}\right), \\
& \tilde{x}=\tilde{k}_{-}+\tilde{d}_{+}=\tilde{d}_{-}+\tilde{k}_{+} \in\left(K_{-}+D_{+}\right) \cap\left(D_{-}+K_{+}\right),
\end{aligned}
$$

and $k_{ \pm}, \tilde{k}_{ \pm} \in K_{ \pm}$and $d_{ \pm}, \tilde{d}_{ \pm} \in D_{ \pm}$. Note that there exist $k_{+}^{\prime} \in K_{+}$and $\tilde{k}_{-}^{\prime} \in K_{-}$ such that $k_{+} R k_{+}^{\prime}$ and $\tilde{k}_{-}^{\prime} R \tilde{k}_{-}$. By linearity of $R$ we obtain

$$
\underbrace{\left(x-k_{+}-\tilde{k}_{-}^{\prime}\right)}_{\in D_{-}} R \underbrace{\left(\tilde{x}-k_{+}^{\prime}-\tilde{k}_{-}\right)}_{\in D_{+}} .
$$

We conclude $d:=x-k_{+}-\tilde{k}_{-}^{\prime} \in D, \tilde{d}:=\tilde{x}-k_{+}^{\prime}-\tilde{k}_{-} \in D$, and $d R \tilde{d}$. This shows that the relations induced on the two quotients in 78 coincide. We complete the proof by showing that $R_{\text {reg }}$ is an isomorphism. Clearly, $\operatorname{dom}\left(R_{\text {reg }}\right)=V_{\text {reg }}=\operatorname{img}\left(R_{\text {reg }}\right)$. We will next show $\operatorname{ker}\left(R_{\text {reg }}\right)=0$. To this end suppose $d R \tilde{d}$, where

$$
d \in D \quad \text { and } \quad \tilde{d}=\tilde{k}_{-}+\tilde{k}_{+} \in\left(K_{-}+K_{+}\right) \cap D
$$


with $\tilde{k}_{ \pm} \in K_{ \pm}$. Note that $\tilde{k}_{-}=\tilde{d}-\tilde{k}_{+} \in K_{-} \cap D_{+}$. Thus there exists $k_{-} \in K_{-} \cap D_{+}$ such that $k_{-} R \tilde{k}_{-}$. By linearity of $R$, we get $\left(d-k_{-}\right) R \tilde{k}_{+}$, whence $d-k_{-} \in K_{+}$and thus $d \in K_{-}+K_{+}$. This shows $\operatorname{ker}\left(R_{\text {reg }}\right)=0$. Analogously, we have $\operatorname{mul}\left(R_{\text {reg }}\right)=0$. In view of Lemma 8.1 we conclude that $R_{\text {reg }}$ is an isomorphism of modules.

We will now specialize to linear relations on finite dimensional vector spaces and provide another description of $V_{\text {reg }}$ in this case. Consider the category whose objects are finite dimensional vector spaces $V$ equipped with a linear relation $R: V \rightsquigarrow V$ and whose morphisms are linear maps $\psi: V \rightarrow W$ such that for all $x, y \in V$ with $x R y$ we also have $\psi(x) Q \psi(y)$, where $W$ is another finite dimensional vector space with linear relation $Q: W \rightsquigarrow W$. It is readily checked that this is an abelian category. By the Krull-Remak-Schmidt theorem, every linear relation on a finite dimensional vector space can therefore be decomposed into a direct sum of indecomposable ones, $R \cong R_{1} \oplus \cdots \oplus R_{N}$, where the factors are unique up to permutation and isomorphism. The decomposition itself, however, is not canonical.

Proposition 8.3. Let $R: V \rightsquigarrow V$ be a linear relation on a finite dimensional vector space over a field, and let $R \cong R_{1} \oplus \cdots \oplus R_{N}$ denote a decomposition into indecomposable linear relations. Then $R_{\mathrm{reg}}$ is isomorphic to the direct sum of factors $R_{i}$ whose relations are linear isomorphisms.

Proof. Since the definition of $R_{\text {reg }}$ is a natural one, we clearly have

$$
R_{\text {reg }} \cong\left(R_{1}\right)_{\text {reg }} \oplus \cdots \oplus\left(R_{N}\right)_{\text {reg }} .
$$

Consequently, it suffices to show the following two assertions:

(a) If $R: V \rightsquigarrow V$ is an isomorphism of vector spaces, then $V_{\text {reg }}=V$ and $R_{\text {reg }}=R$.

(b) If $R: V \rightsquigarrow V$ is an indecomposable linear relation on a finite dimensional vector space which is not a linear isomorphism, then $V_{\text {reg }}=0$.

The first statement is obvious, in this case we have $K_{-}=K_{+}=0$ and $D=D_{-}=$ $D_{+}=V$. To see the second assertion, note that an indecomposable linear relation $R \subseteq V \times V$ gives rise to an indecomposable representation $R_{\rightarrow}^{\rightarrow} V$ of the quiver $G_{2}$. Since $R$ is not an isomorphism, the quiver representation has to be of the bar code type. Using the explicit descriptions of the bar code representations, it is straight forward to conclude $V_{\text {reg }}=0$.

In the subsequent discussion we will also make use of the following result:

Proposition 8.4. Suppose $R: V \rightsquigarrow V$ is a linear relation on a finite dimensional vector space. Then:

$$
\begin{gathered}
D_{+}=D+K_{+}, \quad D_{-}=K_{-}+D, \quad \text { and } \\
K_{-} \cap D_{+}=K_{-} \cap K_{+}=D_{-} \cap K_{+} .
\end{gathered}
$$

For the proof we first establish two lemmas.

Lemma 8.5. Suppose $R: V \rightsquigarrow W$ is a linear relation between vector spaces such that $\operatorname{dim} V=\operatorname{dim} W<\infty$. Then the following are equivalent:

(a) $R$ is an isomorphism.

(b) $\operatorname{dom}(R)=V$ and $\operatorname{ker}(R)=0$.

(c) $\operatorname{img}(R)=W$ and $\operatorname{mul}(R)=0$. 
Proof. This follows immediately from the dimension formula

$$
\operatorname{dim} \operatorname{dom}(R)+\operatorname{dim} \operatorname{mul}(R)=\operatorname{dim}(R)=\operatorname{dim} \operatorname{img}(R)+\operatorname{dim} \operatorname{ker}(R)
$$

and Lemma 8.1 .

Lemma 8.6. If $V$ is finite dimensional, then the composition of relations

$$
D_{+} / K_{+} \stackrel{\pi^{\dagger}}{\rightsquigarrow} D_{+} \stackrel{\iota}{\rightsquigarrow} V \stackrel{R^{k}}{\rightsquigarrow} V \stackrel{\iota^{\dagger}}{\rightsquigarrow} D_{+} \stackrel{\pi}{\rightsquigarrow} D_{+} / K_{+},
$$

is a linear isomorphism, for every $k \geq 0$, where $\iota$ and $\pi$ denote the canonical inclusion and projection, respectively. Analogously, the relation induced by $R^{k}$ on $D_{-} / K_{-}$is an isomorphism, for all $k \geq 0$. Moreover, for sufficiently large $k$,

$$
D_{-}=\operatorname{img}\left(R^{k}\right) \quad \text { and } \quad D_{+}=\operatorname{dom}\left(R^{k}\right) .
$$

Proof. One readily verifies $\operatorname{dom}\left(\pi \iota^{\dagger} R^{k} \iota \pi^{\dagger}\right)=D_{+} / K_{+}$and $\operatorname{ker}\left(\pi \iota^{\dagger} R^{k} \iota \pi^{\dagger}\right)=0$. The first assertion thus follows from Lemma 8.5 above. Considering $R^{\dagger}$ we obtain the second statement. Clearly, $\operatorname{dom}\left(R^{k}\right) \supseteq \operatorname{dom}\left(R^{k+1}\right)$, for all $k \geq 0$. Since $V$ is finite dimensional, we must have $\operatorname{dom}\left(R^{k}\right)=\operatorname{dom}\left(R^{k+1}\right)$, for sufficiently large $k$. Given $v \in \operatorname{dom}\left(R^{k}\right)$, we thus find $v_{1} \in \operatorname{dom}\left(R^{k}\right)$ such that $v R v_{1}$. Proceeding inductively, we construct $v_{i} \in \operatorname{img}\left(R^{k}\right)$ such that $v R v_{1} R v_{2} R \cdots$, whence $v \in D_{+}$. This shows $\operatorname{dom}\left(R^{k}\right) \subseteq D_{+}$, for sufficiently large $k$. As the converse inclusion is obvious we get $D_{+}=\operatorname{dom}\left(R^{k}\right)$. Considering $R^{\dagger}$, we obtain the last statement.

Proof of Proposition 8.4. From Lemma 8.6 we get $\operatorname{img}\left(\pi \iota^{\dagger} R^{k}\right)=D_{+} / K_{+}$, whence $D_{+} \subseteq \operatorname{img}\left(R^{k}\right)+K_{+}$, for every $k \geq 0$, and thus $D_{+} \subseteq D_{-}+K_{+}$. This implies $D_{+}=$ $D+K_{+}$. Considering $R^{\dagger}$ we obtain the other equality in 79 . From Lemma 8.6 we also get $\operatorname{mul}\left(\pi \iota^{\dagger} R^{k}\right)=0$, whence $\operatorname{mul}\left(R^{k}\right) \cap D_{+} \subseteq K_{+}$, for every $k \geq 0$. This gives $K_{-} \cap D_{+}=K_{-} \cap K_{+}$. Considering $R^{\dagger}$ we get the other equality in 80 .

Let us describe the regular part of a linear transformation $\varphi: V \rightarrow V$ on a finite dimensional vector space $V$ more explicitly. In this case, we clearly have $K_{-}=0, K_{+}=\bigcup_{n} \operatorname{ker} \varphi^{n}, D_{+}=V$ and $D=D_{-}=\bigcap_{n} \operatorname{img} \varphi^{n}$. Moreover, $\left(K_{-}+K_{+}\right) \cap D=0$ according to 80$)$ in Proposition 8.4. Hence, the regular part of $\varphi$ coincides with the restriction $\varphi: \bigcap_{n} \operatorname{img} \varphi^{n} \rightarrow \bigcap_{n} \operatorname{img} \varphi^{n}$, see (77). According to Proposition 8.2, the regular part of $\varphi$ can alternatively be described as the induced isomorphism $\varphi_{\text {reg }}: V / \bigcup_{n} \operatorname{ker} \varphi^{n} \rightarrow V / \bigcup_{n} \operatorname{ker} \varphi^{n}$, for we have $V=D_{-}+K_{+}$in view of 79 in Proposition 8.4

The following notation and observation will be used in the appendix. For two linear maps, $A, B: V \rightarrow W$, we let $R(A, B): V \rightsquigarrow V$ denote the linear relation $R(A, B):=\left\{\left(v_{1}, v_{2}\right) \mid A\left(v_{1}\right)=B\left(v_{2}\right)\right\}$.

Observation 8.7. Suppose $A, B: V \rightarrow W$ are two linear maps.

(a) If $A^{\prime}, B^{\prime}: V \rightarrow W^{\prime}$ denote the composition of $A$ and $B$ with an inclusion of vector spaces, $W \subseteq W^{\prime}$, then $R(A, B)=R\left(A^{\prime}, B^{\prime}\right)$.

(b) If $A$ is invertible then $R(A, B)=R\left(\mathrm{Id}, A^{-1} B\right)=R\left(A^{-1} B \text {, Id }\right)^{\dagger}$.

(c) If $A$ is invertible then $R(A, B)_{\mathrm{reg}}=\left(\left(A^{-1} B\right)_{\mathrm{reg}}\right)^{-1}$. 
8.2. Monodromy. Suppose $f: X \rightarrow S^{1}$ is a continuous map and let

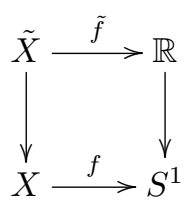

denote the associated infinite cyclic covering. For $r \in \mathbb{R}$ we put $\tilde{X}_{r}=\tilde{f}^{-1}(r)$ and let $H_{*}\left(\tilde{X}_{r}\right)$ denote its singular homology with coefficients in any fixed field. If $r_{1} \leq r_{2}$ we define a linear relation

$$
B_{r_{1}}^{r_{2}}: H_{*}\left(\tilde{X}_{r_{1}}\right) \rightsquigarrow H_{*}\left(\tilde{X}_{r_{2}}\right)
$$

by declaring $a_{1} \in H_{*}\left(\tilde{X}_{r_{1}}\right)$ to be in relation with $a_{2} \in H_{*}\left(\tilde{X}_{r_{2}}\right)$ iff their images in $H_{*}\left(\tilde{X}_{\left[r_{1}, r_{2}\right]}\right)$ coincide, where $\tilde{X}_{\left[r_{1}, r_{2}\right]}=\tilde{f}^{-1}\left(\left[r_{1}, r_{2}\right]\right)$.

If $r_{1} \leq r_{2} \leq r_{3}$ we clearly have $B_{r_{2}}^{r_{3}} B_{r_{1}}^{r_{2}} \subseteq B_{r_{1}}^{r_{3}}$. To formulate a criterion which guarantees equality of relations, $B_{r_{2}}^{r_{3}} B_{r_{1}}^{r_{2}}=B_{r_{1}}^{r_{3}}$, we introduce the following notation: A number $r \in \mathbb{R}$ is called tame value if, for every $\varepsilon>0$, there exists a neighborhood $U$ of $\tilde{X}_{r}$ in $\tilde{X}_{[r-\varepsilon, r+\varepsilon]}$ such that each of the inclusions $\tilde{X}_{r} \subseteq U, \tilde{X}_{[r-\varepsilon, r]} \cap U \subseteq U$, and $\tilde{X}_{[r, r+\varepsilon]} \cap U \subseteq U$, induces isomorphisms in homology. The crucial point is that in this case the triad $\left(\tilde{X}_{[r-\varepsilon, r+\varepsilon]} ; \tilde{X}_{[r, r+\varepsilon]}, \tilde{X}_{[r-\varepsilon, r]}\right)$ gives rise to a long exact Mayer-Vietoris sequence. Note that for a tame map as considered in Section 11, all values are tame.

Lemma 8.8. Suppose $r_{1} \leq r_{2} \leq r_{3}$ and assume $r_{2}$ is a tame value. Then, as linear relations, $B_{r_{2}}^{r_{3}} B_{r_{1}}^{r_{2}}=B_{r_{1}}^{r_{3}}$.

Proof. Since $r_{2}$ is a tame value, we have an exact Mayer-Vietoris sequence,

$$
H_{*}\left(\tilde{X}_{r_{2}}\right) \rightarrow H_{*}\left(\tilde{X}_{\left[r_{1}, r_{2}\right]}\right) \oplus H_{*}\left(\tilde{X}_{\left[r_{2}, r_{3}\right]}\right) \rightarrow H_{*}\left(\tilde{X}_{\left[r_{1}, r_{3}\right]}\right) .
$$

This immediately gives $B_{r_{2}}^{r_{3}} B_{r_{1}}^{r_{2}} \supseteq B_{r_{1}}^{r_{3}}$. As the converse inclusion, $B_{r_{2}}^{r_{3}} B_{r_{1}}^{r_{2}} \subseteq B_{r_{1}}^{r_{3}}$, is obvious, the lemma follows.

Fix a tame value $\theta \in S^{1}$ of $f$ and a lift $\tilde{\theta} \in \mathbb{R}, e^{\mathbf{i} \tilde{\theta}}=\theta$. Using the projection $\tilde{X} \rightarrow X$, we may canonically identify $\tilde{X}_{\tilde{\theta}}=X_{\theta}=f^{-1}(\theta)$. Moreover, let $\tau: \tilde{X} \rightarrow \tilde{X}$ denote the fundamental deck transformation, i.e. $\tilde{f} \circ \tau=\tilde{f}+2 \pi$. Note that $\tau$ induces homeomorphisms between levels, $\tau: \tilde{X}_{r} \rightarrow \tilde{X}_{r+2 \pi}$, and define a linear relation

$$
R_{\theta}: H_{*}\left(X_{\theta}\right) \rightsquigarrow H_{*}\left(X_{\theta}\right)
$$

as the composition

$$
H_{*}\left(X_{\theta}\right)=H_{*}\left(\tilde{X}_{\tilde{\theta}}\right) \stackrel{B_{\tilde{\theta}}^{\tilde{\theta}+2 \pi}}{\rightsquigarrow} H_{*}\left(\tilde{X}_{\tilde{\theta}+2 \pi}\right) \stackrel{\tau_{*}^{\dagger}}{\rightsquigarrow} H_{*}\left(\tilde{X}_{\tilde{\theta}}\right)=H_{*}\left(X_{\theta}\right) .
$$

In other words, for $a, b \in H_{*}\left(X_{\theta}\right)$ we have $a R b$ iff $a B_{\tilde{\theta}}^{\tilde{\theta}+2 \pi}\left(\tau_{*} b\right)$, i.e. iff $a$ and $\tau_{*} b$ coincide in $H_{*}\left(\tilde{X}_{[\tilde{\theta}, \tilde{\theta}+2 \pi]}\right)$. Particularly, we have:

Lemma 8.9. If $a, b \in H_{*}\left(X_{\theta}\right)$ and $a R b$, then $a=\tau_{*} b$ in $H_{*}(\tilde{X})$.

We will continue to use the notation $K_{ \pm}, D_{ \pm}$, and $R_{\text {reg }}$ introduced in the previous section for this relation $R$ on $H_{*}\left(X_{\theta}\right)$. Particularly, its regular part,

$$
R_{\text {reg }}: H_{*}\left(X_{\theta}\right)_{\text {reg }} \rightarrow H_{*}\left(X_{\theta}\right)_{\text {reg }},
$$

is a module automorphism. 
Lemma 8.10. We have:

$$
\begin{aligned}
& K_{+}=\operatorname{ker}\left(H_{*}\left(X_{\theta}\right) \rightarrow H_{*}\left(\tilde{X}_{[\tilde{\theta}, \infty)}\right)\right) \\
& K_{-}=\operatorname{ker}\left(H_{*}\left(X_{\theta}\right) \rightarrow H_{*}\left(\tilde{X}_{(-\infty, \tilde{\theta}]}\right)\right)
\end{aligned}
$$

Both maps are induced by the canonical inclusion $X_{\theta}=\tilde{X}_{\tilde{\theta}} \rightarrow \tilde{X}$.

Proof. We will only show the first equality, the other one can be proved along the same lines. To see the inclusion $K_{+} \subseteq \operatorname{ker}\left(H_{*}\left(X_{\theta}\right) \rightarrow H_{*}\left(\tilde{X}_{[\tilde{\theta}, \infty)}\right)\right)$, let $a \in$ $K_{+}$. Hence, there exist $a_{k} \in H_{*}\left(X_{\theta}\right)$, almost all of which vanish, such that $a R a_{1} R a_{2} R \cdots$. In $H_{*}\left(\tilde{X}_{[\tilde{\theta}, \tilde{\theta}+2 \pi]}\right)$, we thus have:

$$
a=\tau_{*} a_{1}, \quad a_{1}=\tau_{*} a_{2}, \quad a_{2}=\tau_{*} a_{3}, \quad \ldots
$$

In $H_{*}\left(\tilde{X}_{[\tilde{\theta}, \infty)}\right)$, we obtain:

$$
a=\tau_{*} a_{1}=\tau_{*}^{2} a_{2}=\tau_{*}^{3} a_{3}=\cdots
$$

Since some $a_{k}$ have to be zero, we conclude that $a$ vanishes in $H_{*}\left(\tilde{X}_{[\tilde{\theta}, \infty)}\right)$.

To see the converse inclusion, $K_{+} \supseteq \operatorname{ker}\left(H_{*}\left(\tilde{X}_{\theta}\right) \rightarrow H_{*}\left(\tilde{X}_{[\tilde{\theta}, \infty)}\right)\right)$, set

$$
U:=\bigsqcup_{0 \leq k \text { even }} \tilde{X}_{[\tilde{\theta}+2 \pi k, \tilde{\theta}+2 \pi(k+1)]}, \quad V:=\bigsqcup_{1 \leq k \text { odd }} \tilde{X}_{[\tilde{\theta}+2 \pi k, \tilde{\theta}+2 \pi(k+1)]}
$$

and note that $U \cup V=\tilde{X}_{[\tilde{\theta}, \infty)}$, as well as $U \cap V=\bigsqcup_{k \in \mathbb{N}} \tilde{X}_{\tilde{\theta}+2 \pi k}$. Since $\theta$ is a tame value, we have an exact Mayer-Vietoris sequence

$$
\bigoplus_{k \in \mathbb{N}} H_{*}\left(\tilde{X}_{\tilde{\theta}+2 \pi k}\right)=H_{*}\left(\bigsqcup_{k \in \mathbb{N}} \tilde{X}_{\tilde{\theta}+2 \pi k}\right) \rightarrow H_{*}(U) \oplus H_{*}(V) \rightarrow H_{*}\left(\tilde{X}_{[\tilde{\theta}, \infty)}\right) .
$$

For $b \in \operatorname{ker}\left(H_{*}\left(X_{\theta}\right) \rightarrow H_{*}\left(\tilde{X}_{[\tilde{\theta}, \infty)}\right)\right)$ we thus find $b_{k} \in H_{*}\left(\tilde{X}_{\tilde{\theta}+2 \pi k}\right)$, almost all of which vanish, such that:

$$
\begin{aligned}
b & =b_{1} \in H_{*}\left(\tilde{X}_{[\tilde{\theta}, \tilde{\theta}+2 \pi]}\right) \\
b_{1}+b_{2} & =0 \in H_{*}\left(\tilde{X}_{[\tilde{\theta}+2 \pi, \tilde{\theta}+4 \pi]}\right) \\
b_{2}+b_{3} & =0 \in H_{*}\left(\tilde{X}_{[\tilde{\theta}+4 \pi, \tilde{\theta}+6 \pi]}\right)
\end{aligned}
$$

Putting $c_{k}:=(-1)^{k-1} \tau_{*}^{-k} b_{k} \in H_{*}\left(\tilde{X}_{\tilde{\theta}}\right)$, we obtain the following equalities in $H_{*}\left(\tilde{X}_{[\tilde{\theta}, \tilde{\theta}+2 \pi]}\right):$

$$
b=\tau_{*} c_{1}, \quad c_{1}=\tau_{*} c_{2}, \quad c_{2}=\tau_{*} c_{3}, \quad \ldots
$$

In other words, we have the relations $b R c_{1} R c_{2} R c_{3} R \cdots$. Since some $c_{k}$ has to be zero, we conclude $b \in K_{+}$, whence the lemma.

Introduce the upwards Novikov complex as a projective limit of relative singular chain complexes,

$$
C_{*}^{\mathrm{Nov},+}(\tilde{X}):={\underset{\lim }{r}}_{\lim _{*}}\left(\tilde{X}, \tilde{X}_{[r, \infty)}\right),
$$

and let $H_{*}^{\mathrm{Nov},+}(\tilde{X})$ denote its homology. Alternatively, $C_{*}^{\mathrm{Nov},+}(\tilde{X})$ can be described as the chain complex of formal, possibly infinite, linear combinations of singular simplices in $\tilde{X}$ such that the number of simplices intersecting $\tilde{X}_{(-\infty, r]}$ is finite, for all real values $r$. Analogously, we define a downwards Novikov complex $C_{*}^{\text {Nov, }-}(\tilde{X})=$ 
$\lim _{r} C_{*}\left(\tilde{X}, \tilde{X}_{(-\infty, r]}\right)$ and the corresponding homology, $H_{*}^{\mathrm{Nov},-}(\tilde{X})$. We will also use similar notation for subsets of $\tilde{X}$.

Lemma 8.11. We have:

$$
\begin{aligned}
& D_{+}=\operatorname{ker}\left(H_{*}\left(X_{\theta}\right) \rightarrow H_{*}^{\mathrm{Nov},+}\left(\tilde{X}_{[\tilde{\theta}, \infty)}\right)\right) \\
& D_{-}=\operatorname{ker}\left(H_{*}\left(X_{\theta}\right) \rightarrow H_{*}^{\mathrm{Nov},-}\left(\tilde{X}_{(-\infty, \tilde{\theta}]}\right)\right)
\end{aligned}
$$

Both maps are induced by the canonical inclusion $X_{\theta}=\tilde{X}_{\tilde{\theta}} \rightarrow \tilde{X}$.

Proof. Using the exact Mayer-Vietoris sequence

$\prod_{k \in \mathbb{N}} H_{*}\left(\tilde{X}_{\tilde{\theta}+2 \pi k}\right)=H_{*}^{\mathrm{Nov},+}\left(\bigsqcup_{k \in \mathbb{N}} \tilde{X}_{\tilde{\theta}+2 \pi k}\right) \rightarrow H_{*}^{\mathrm{Nov},+}(U) \oplus H_{*}^{\mathrm{Nov},+}(V) \rightarrow H_{*}^{\mathrm{Nov},+}\left(\tilde{X}_{[\tilde{\theta}, \infty)}\right)$,

this can be proved along the same lines as Lemma 8.10.

Let us introduce a complex

$$
C_{*}^{\text {l.f. }}(\tilde{X}):={\underset{\varliminf}{r}}_{\lim } C_{*}\left(\tilde{X}, \tilde{X}_{(-\infty,-r]} \cup \tilde{X}_{[r, \infty)}\right)
$$

and denote its homology by $H_{*}^{\text {l.f. }}(\tilde{X})$. If $f$ is proper, this is the complex of locally finite singular chains.

Lemma 8.12. We have:

$$
\begin{aligned}
& K_{-}+K_{+}=\operatorname{ker}\left(H_{*}\left(X_{\theta}\right) \rightarrow H_{*}(\tilde{X})\right) \\
& K_{-}+D_{+}=\operatorname{ker}\left(H_{*}\left(X_{\theta}\right) \rightarrow H_{*}^{\mathrm{Nov},+}(\tilde{X})\right) \\
& D_{-}+K_{+}=\operatorname{ker}\left(H_{*}\left(X_{\theta}\right) \rightarrow H_{*}^{\text {Nov },-}(\tilde{X})\right) \\
& D_{-}+D_{+}=\operatorname{ker}\left(H_{*}\left(X_{\theta}\right) \rightarrow H_{*}^{\text {l.f. }}(\tilde{X})\right)
\end{aligned}
$$

All maps are induced by the canonical inclusion $X_{\theta}=\tilde{X}_{\tilde{\theta}} \rightarrow \tilde{X}$.

Proof. The first statement follows from the exact Mayer-Vietoris sequence

$$
H_{*}\left(\tilde{X}_{\tilde{\theta}}\right) \rightarrow H_{*}\left(\tilde{X}_{(-\infty, \tilde{\theta}]}\right) \oplus H_{*}\left(\tilde{X}_{[\tilde{\theta}, \infty)}\right) \rightarrow H_{*}(\tilde{X})
$$

and Lemma 8.10 The second assertion follows from the exact Mayer-Vietoris sequence

$$
H_{*}\left(\tilde{X}_{\tilde{\theta}}\right) \rightarrow H_{*}\left(\tilde{X}_{(-\infty, \tilde{\theta}]}\right) \oplus H_{*}^{\mathrm{Nov},+}\left(\tilde{X}_{[\tilde{\theta}, \infty)}\right) \rightarrow H_{*}^{\mathrm{Nov},+}(\tilde{X})
$$

and Lemma 8.10 and 8.11 . Similarly, one can check the third equality. To see the last statement we use the exact Mayer-Vietoris sequence

$$
H_{*}\left(\tilde{X}_{\tilde{\theta}}\right) \rightarrow H_{*}^{\mathrm{Nov},-}\left(\tilde{X}_{(-\infty, \tilde{\theta}]}\right) \oplus H_{*}^{\mathrm{Nov},+}\left(\tilde{X}_{[\tilde{\theta}, \infty)}\right) \rightarrow H_{*}^{\text {l.f. }}(\tilde{X})
$$

and Lemma 8.11 .

Lemma 8.13. We have

$$
\operatorname{ker}\left(H_{*}(\tilde{X}) \rightarrow H_{*}^{\mathrm{Nov},-}(\tilde{X}) \oplus H_{*}^{\mathrm{Nov},+}(\tilde{X})\right) \subseteq \operatorname{img}\left(H_{*}\left(\tilde{X}_{\tilde{\theta}}\right) \rightarrow H_{*}(\tilde{X})\right),
$$

where all maps are induced by the tautological inclusions. 
Proof. This follows from the following commutative diagram of exact Mayer-Vietoris sequences:

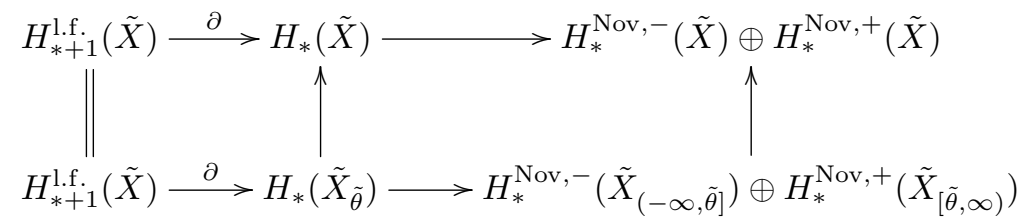

A similar argument was used in [24, Lemma 2.5].

Theorem 8.14. The inclusion $\iota: X_{\theta}=\tilde{X}_{\tilde{\theta}} \rightarrow \tilde{X}$ induces a canonical isomorphism

$$
H_{*}\left(X_{\theta}\right)_{\mathrm{reg}}=\frac{D}{\left(K_{-}+K_{+}\right) \cap D} \stackrel{\cong}{\rightarrow} \operatorname{ker}\left(H_{*}(\tilde{X}) \rightarrow H_{*}^{\mathrm{Nov},-}(\tilde{X}) \oplus H_{*}^{\mathrm{Nov},+}(\tilde{X})\right),
$$

intertwining $R_{\mathrm{reg}}$ with the monodromy isomorphism induced by the deck transformation $\tau: \tilde{X} \rightarrow \tilde{X}$ on the right hand side. Moreover, working with coefficients in a field, and assuming that $H_{*}\left(X_{\theta}\right)$ is finite dimensional, the common kernel on the right hand side above coincides with

$$
\operatorname{ker}\left(H_{*}(\tilde{X}) \rightarrow H_{*}^{\mathrm{Nov},-}(\tilde{X})\right)=\operatorname{ker}\left(H_{*}(\tilde{X}) \rightarrow H_{*}^{\mathrm{Nov},+}(\tilde{X})\right) .
$$

Particularly, in this case the latter two kernels are finite dimensional too.

Proof. It follows immediately from Lemma 8.12 and 8.13 that $\iota_{*}: H_{*}\left(X_{\theta}\right) \rightarrow H_{*}(\tilde{X})$ induces an isomorphism

$$
\frac{\left(K_{-}+D_{+}\right) \cap\left(D_{-}+K_{+}\right)}{K_{-}+K_{+}} \cong \operatorname{mer}\left(H_{*}(\tilde{X}) \rightarrow H_{*}^{\mathrm{Nov},-}(\tilde{X}) \oplus H_{*}^{\mathrm{Nov},+}(\tilde{X})\right) .
$$

In view of Lemma 8.9, this isomorphism intertwines the isomorphism induced by $R$ on the left hand side, with the monodromy isomorphism on the right hand side. Combining this with Proposition 8.2 we obtain the first assertion. For the second statement it suffices to show

$$
\operatorname{ker}\left(H_{*}(\tilde{X}) \rightarrow H_{*}^{\mathrm{Nov},+}(\tilde{X})\right) \subseteq \operatorname{ker}\left(H_{*}(\tilde{X}) \rightarrow H_{*}^{\mathrm{Nov},-}(\tilde{X}) \oplus H_{*}^{\mathrm{Nov},+}(\tilde{X})\right),
$$

as the converse inclusion is obvious, and the corresponding statement for the downward Novikov homology can be derived analogously. To this end, suppose $a \in \operatorname{ker}\left(H_{*}(\tilde{X}) \rightarrow H_{*}^{\mathrm{Nov},+}(\tilde{X})\right)$. Then there exists $k$ such that $\tau_{*}^{k} a$ is contained in the image of $H_{*}\left(\tilde{X}_{(-\infty, \tilde{\theta}]}\right) \rightarrow H_{*}(\tilde{X})$. Using the exact Mayer-Vietoris sequence

$$
H_{*}\left(\tilde{X}_{\tilde{\theta}}\right) \rightarrow H_{*}\left(\tilde{X}_{(-\infty, \tilde{\theta}]}\right) \oplus H_{*}^{\mathrm{Nov},+}\left(\tilde{X}_{[\tilde{\theta}, \infty)}\right) \rightarrow H_{*}^{\mathrm{Nov},+}(\tilde{X})
$$

we conclude, that $\tau_{*}^{k} a$ is contained in the image of $H_{*}\left(\tilde{X}_{\tilde{\theta}}\right) \rightarrow H_{*}(\tilde{X})$. Thus $\tau_{*}^{k} a$ is contained in $\iota_{*}\left(D_{+}\right)$, see Lemma 8.12 . Since $H_{*}\left(X_{\theta}\right)$ is assumed to be a finite dimensional vector space, we have $\iota_{*}\left(D_{-}\right)=\iota_{*}(D)=\iota_{*}\left(D_{+}\right)$, see 79 . Using Lemma 8.12 we thus conclude $\tau_{*}^{k} a$ is contained in the kernel on the right hand side of 82 . Since this common kernel is invariant under the isomorphism $\tau_{*}: H_{*}(\tilde{X}) \rightarrow$ $H_{*}(X)$, we conclude that $a$ has to be contained in the common kernel too, whence the theorem.

We conclude this section with a proof of Theorem 1.4. Suppose $X$ is a compact ANR and let $f: X \rightarrow S^{1}$ be a tame map as in Section 1. Fix regular and critical 
angles, $0<t_{1}<\theta_{1}<\cdots<t_{m}<\theta_{m} \leq 2 \pi$, and consider the associated $G_{2 m^{-}}$ representation $\rho_{r}=\left\{V_{i}, \alpha_{i}, \beta_{i}\right\}$, see Section 3 . Note that the linear relation $R_{r}^{\theta}$ on $H_{r}\left(X_{\theta}\right)$ introduced in Section 1 is just the degree $r$ part of the relation considered in this section, see (81). From Lemma 8.8 we immediately obtain:

Lemma 8.15. The following equalities of relations on $H_{r}\left(X_{\theta}\right)$ hold true:

(a) If $\theta=\theta_{i}$, then $R_{r}^{\theta}=\alpha_{i} \beta_{i-1}^{\dagger} \alpha_{i-1} \cdots \beta_{1}^{\dagger} \alpha_{1} \beta_{m}^{\dagger} \alpha_{m} \cdots \alpha_{i+2} \beta_{i+1}^{\dagger} \alpha_{i+1} \beta_{i}^{\dagger}$.

(b) If $\theta=t_{i}$, then $R_{r}^{\theta}=\beta_{i-1}^{\dagger} \alpha_{i-1} \beta_{i-2}^{\dagger} \cdots \beta_{1}^{\dagger} \alpha_{1} \beta_{m}^{\dagger} \alpha_{m} \cdots \beta_{i+1}^{\dagger} \alpha_{i+1} \beta_{i}^{\dagger} \alpha_{i}$.

Lemma 8.16. Suppose $\rho=\left\{V_{i}, \alpha_{i}, \beta_{i}\right\}$ is a $G_{2 m}$-representation with Jordan blocks $\bigoplus_{J \in \mathcal{J}} T(J)$. Then, for all $1 \leq i \leq m$, the following hold true:

(a) $\left(\alpha_{i} \beta_{i-1}^{\dagger} \alpha_{i-1} \cdots \beta_{1}^{\dagger} \alpha_{1} \beta_{m}^{\dagger} \alpha_{m} \cdots \alpha_{i+2} \beta_{i+1}^{\dagger} \alpha_{i+1} \beta_{i}^{\dagger}\right)_{\text {reg }}$ is conjugate to $\bigoplus_{J \in \mathcal{J}} T(J)$.

(b) $\left(\beta_{i-1}^{\dagger} \alpha_{i-1} \beta_{i-2}^{\dagger} \cdots \beta_{1}^{\dagger} \alpha_{1} \beta_{m}^{\dagger} \alpha_{m} \cdots \beta_{i+1}^{\dagger} \alpha_{i+1} \beta_{i}^{\dagger} \alpha_{i}\right)_{\text {reg }}$ is conjugate to $\bigoplus_{J \in \mathcal{J}} T(J)$.

Proof. W.l.o.g. it suffices to consider an indecomposable representation $\rho$. For such a $\rho$, however, the statement follows immediately from the classification of indecomposable representations discussed in Section 2, see also Proposition 8.3.

Combining the preceding two lemmas, we obtain Theorem 1.4

\section{Proof of Theorem 1.7}

Suppose $f: X \rightarrow \mathbb{S}^{1}$ is a tame map. For $0<\theta^{\prime} \leq \theta^{\prime \prime} \leq 2 \pi$ we will use the notation $X_{\left[\theta^{\prime}, \theta^{\prime \prime}\right]}:=f^{-1}\left(\left[\theta^{\prime}, \theta^{\prime \prime}\right]\right)$, and write $X(\theta):=X_{[\theta, \theta]}=f^{-1}(\theta)$.

Let $0<\theta_{1}<\theta_{2}<\cdots<\theta_{N} \leq 2 \pi$ be the collection of all critical values and put $\epsilon(f):=\min \left\{\left|\theta_{i+1}-\theta_{i}\right|: 1 \leq i \leq N\right\}$ where $\theta_{N+1}:=\theta_{1}+2 \pi$. Note that any bar code $I \in \widetilde{\mathcal{B}}_{r}(f)$ has the left end of the form $\theta_{i}+2 \pi k$ and the right end of the form $\theta_{j}+2 \pi k^{\prime}$ where $i, j \in\{1, \ldots, N\}$ and $k, k^{\prime} \in \mathbb{Z}$. Put $l(I):=\theta_{i}$ and $r(I):=\theta_{j}$. The numbers $l(I)$ and $r(I)$ are well defined for barcodes in $\mathcal{B}_{r}(f)$ which can be considered as equivalency classes of elements in $\widetilde{\mathcal{B}}_{r}(f)$.

Proposition 9.1. For any tame map $f: X \rightarrow \mathbb{S}^{1}$ and $0<\epsilon<\epsilon(f)$ we have:

$$
\begin{aligned}
& \operatorname{dim} H_{r}\left(X_{\left[\theta_{i}-\epsilon, \theta_{i}+\epsilon\right]},\right.\left.X\left(\theta_{i}-\epsilon\right)\right) \\
&=\sharp\left\{I \in \mathcal{B}_{r}^{c}(f) \mid l(I)=\theta_{i}\right\}+\sharp\left\{I \in \mathcal{B}_{r-1}^{o}(f) \mid r(I)=\theta_{i}\right\} \\
& \quad+\sharp\left\{I \in \mathcal{B}_{r}^{c o}(f) \mid l(I)=\theta_{i}\right\}+\sharp\left\{I \in \mathcal{B}_{r-1}^{c o}(f) \mid r(I)=\theta_{i}\right\} .
\end{aligned}
$$

Proof. By the long exact homology sequence of the pair $\left(X_{\left[\theta_{i}-\epsilon, \theta_{i}+\epsilon\right]}, X\left(\theta_{i}-\epsilon\right)\right)$,

$$
\begin{aligned}
\operatorname{dim} H_{r}\left(X_{\left[\theta_{i}-\epsilon, \theta_{i}+\epsilon\right]},\right. & \left.X\left(\theta_{i}-\epsilon\right)\right) \\
=\operatorname{dim} & \operatorname{coker}\left(H_{r}\left(X\left(\theta_{i}-\epsilon\right)\right) \stackrel{i_{r}}{\longrightarrow} H_{r}\left(X_{\left[\theta_{i}-\epsilon, \theta_{i}+\epsilon\right]}\right)\right) \\
& +\operatorname{dim} \operatorname{ker}\left(H_{r-1}\left(X\left(\theta_{i}-\epsilon\right)\right) \stackrel{i_{r-1}}{\longrightarrow} H_{r-1}\left(X_{\left[\theta_{i}-\epsilon, \theta_{i}+\epsilon\right]}\right)\right) .
\end{aligned}
$$

According to Proposition 4.2, there exist isomorphisms $\omega_{1}(r)$ and $\omega_{2}(r)$ such that the diagram

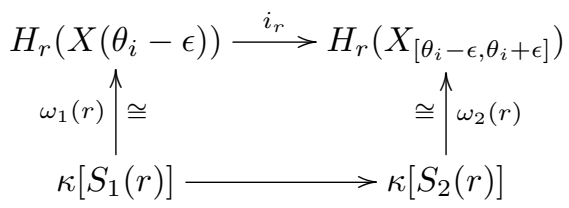


commutes, where

$$
\begin{aligned}
& S_{1}(r)=\left\{I \in \widetilde{\mathcal{B}}_{r}(f) \mid \theta_{i}-\epsilon \in I\right\} \sqcup \widetilde{\mathcal{J}}_{r}(f), \\
& S_{2}(r)=\left\{I \in \widetilde{\mathcal{B}}_{r}(f) \mid \theta_{i} \in I\right\} \sqcup \widetilde{\mathcal{J}}_{r}(f),
\end{aligned}
$$

and the lower horizontal arrow in the diagram denotes the canonical map associated with the subsets $S_{1}(r)$ and $S_{2}(r)$ of $\widetilde{\mathcal{B}}_{r}(f) \sqcup \widetilde{\mathcal{J}}_{r}(f)$. From this description one readily obtains $\operatorname{coker}\left(i_{r}\right) \cong \kappa\left[S_{3}(r)\right]$ and $\operatorname{ker}\left(i_{r-1}\right) \cong \kappa\left[S_{4}(r)\right]$, where

$$
\begin{aligned}
& S_{3}(r)=\left\{I \in \mathcal{B}_{r}^{c} \mid l(I)=\theta_{i}\right\} \sqcup\left\{I \in \mathcal{B}_{r}^{c o} \mid l(I)=\theta_{i}\right\}, \\
& S_{4}(r)=\left\{I \in \mathcal{B}_{r-1}^{o} \mid r(I)=\theta_{i}\right\} \sqcup\left\{I \in \mathcal{B}_{r-1}^{c o} \mid r(I)=\theta_{i}\right\},
\end{aligned}
$$

and thus

$$
\begin{aligned}
& \operatorname{dim} \operatorname{coker}\left(i_{r}\right)=\sharp\left\{I \in \mathcal{B}_{r}^{c} \mid l(I)=\theta_{i}\right\}+\sharp\left\{I \in \mathcal{B}_{r}^{c o} \mid l(I)=\theta_{i}\right\}, \\
& \operatorname{dim} \operatorname{ker}\left(i_{r-1}\right)=\sharp\left\{I \in \mathcal{B}_{r-1}^{o} \mid r(I)=\theta_{i}\right\}+\sharp\left\{I \in \mathcal{B}_{r-1}^{c o} \mid r(I)=\theta_{i}\right\} .
\end{aligned}
$$

Combining these equations with 83 we obtain the proposition.

Let $M$ be a closed manifold of dimension $n$, and suppose $f: M \rightarrow \mathbb{S}^{1}$ is a Morse map, i.e., all critical points are non-degenerated. Let $\mathcal{X}(f)$ denote the set of critical points of $f$. For $r=0, \ldots, n$ and $i=1, \ldots, N$ let

$$
\mathcal{X}_{r, i}(f):=\left\{x \in \mathcal{X}(f): \operatorname{ind}(x)=r \text { and } f(x)=\theta_{i}\right\}
$$

denote the set of critical points of Morse index $r$ corresponding to the critical value $\theta_{i}$. Moreover, put $c_{r, i}:=\sharp \mathcal{X}_{r, i}$.

Recall that the Morse Lemma, see [26, Lemma 2.2], asserts that for every nondegenerate critical point $x$ of $f$ there exists an open neighborhood $U_{x}$ of 0 in $\mathbb{R}^{n}$ and a diffeomorphism onto its image, $\varphi_{x}: U_{x} \rightarrow M$, such that $\varphi_{x}(0)=x$ and

$$
f\left(\varphi_{x}\left(t_{1}, \ldots, t_{n}\right)\right)=f(x)-t_{1}^{2}-\cdots-t_{k}^{2}+t_{k+1}^{2}+\cdots+t_{n}^{2}
$$

holds for all $\left(t_{1}, \ldots, t_{n}\right) \in U_{x}$, where $k=\operatorname{ind}_{f}(x)$. In particular, $\mathcal{X}(f)$ is finite, for $M$ is assumed to be compact. We fix Morse coordinates $\varphi_{x}: U_{x} \rightarrow M$ as above for every critical point $x \in \mathcal{X}(f)$. Moreover, we assume $0<\epsilon<\epsilon(f)^{17}$ is sufficiently small such that $D^{n}(\epsilon):=\left\{\left(t_{1}, \ldots, t_{n}\right): \sum_{i+1}^{n} t_{i}^{2} \leq \epsilon\right\} \subseteq U_{x}$ for all $x \in \mathcal{X}(f)$.

Proposition 9.2 (Morse theorem). If $\epsilon>0$ is sufficiently small, then

$$
\operatorname{dim} H_{r}\left(M_{\left[\theta_{i}-\epsilon, \theta_{i}+\epsilon\right]}, M\left(\theta_{i}-\epsilon\right)\right)=c_{r, i}
$$

for every $r=0, \ldots, n$ and all $i=1, \ldots, N$.

The proof of this proposition can be found in any book in Morse theory, see for instance [26, Section $\S 5]$. The idea is simple. For every critical point $x \in \mathcal{X}(f)$ one defines $B_{x}:=\varphi_{x}\left(D^{\text {ind }(x)}(\epsilon)\right)$, where $D^{k}(\epsilon):=\left\{\left(t_{1}, \ldots, t_{k}, 0, \ldots, 0\right): \sum_{i=1}^{k} t_{i}^{2} \leq \epsilon\right\}$. For each $i=1, \ldots, N$ one considers

$$
X(i):=M\left(\theta_{i}-\epsilon\right) \cup \bigcup_{x \in \mathcal{X}(f): f(x)=\theta_{i}} B_{x} \subseteq M_{\left[\theta_{i}-\epsilon, \theta_{i}+\epsilon\right]} .
$$

\footnotetext{
${ }^{17}$ A Morse map is tame when $M$ is compact.
} 
As in [26, Section $\S 3]$ one can verify that $M_{\left[\theta_{i}-\epsilon, \theta_{i}+\epsilon\right]}$ retracts by deformation to $X(i)$. The deformation is obtained using the flow of the gradient vector field $-\operatorname{grad}_{g}(f)$ where $g$ is a conveniently choosen Riemannian metric. Consequently,

$$
\begin{aligned}
H_{r}\left(M_{\left[\theta_{i}-\epsilon, \theta_{i}+\epsilon\right]}, M\left(\theta_{i}-\epsilon\right)\right)=H_{r}(X(i), & \left.M\left(\theta_{i}-\epsilon\right)\right) \\
& =\bigoplus_{x \in \mathcal{X}_{r, i}(f)} H_{r}\left(D_{x}^{r}(\epsilon), \partial D_{x}^{r}(\varepsilon)\right) \cong \kappa^{c_{r, i}} .
\end{aligned}
$$

From Propositions 9.1 and 9.2 we get

$$
c_{r}(f)=\sum_{i=1}^{N} c_{r, i}=\sharp \mathcal{B}_{r}^{c}(f)+\sharp \mathcal{B}_{r-1}^{o}(f)+\sharp \mathcal{B}_{r}^{c o}(f)+\sharp \mathcal{B}_{r-1}^{c o}(f) .
$$

Combining this with Theorem 1.1 (a), we obtain the statement for angle-vcalued maps in Theorem 1.7. A real-valued map can be viewed as an angle-valued map after composition with an embedding of $\mathbb{R}$ in $\mathbb{S}^{1}$. In this case the Novikov-Betti numbers coincide with the Betti numbers, whence the statement for real-valued maps in Theorem 1.7 follows from the statement for angle-valued maps.

\section{Appendix A. An example}

Consider the space $X$ obtained from $Y$ described in Figure 5 by identifying its right end $Y_{1}$ (a union of three circles) to the left end $Y_{0}$ (a union of three circles) following the map $\phi: Y_{1} \rightarrow Y_{0}$ given by the matrix

$$
\left(\begin{array}{ccc}
3 & 3 & 0 \\
2 & 3 & -1 \\
1 & 2 & 3
\end{array}\right) .
$$

The meaning of this matrix as a map $\phi$ is the following: circle (1) is divided in 6 parts, circle (2) in 8 parts and and circle (3) in 4 parts; the first three parts of circle (1) wrap clockwise around circle (1) to cover it three times, the next two wrap clockwise around circle (2) to cover it twice and around circle three to cover it three times. Similarly circle (2) and (3) wrap over circles (1), (2) and (3) as indicated by the matrix. The first part of circle (3) wraps counterclockwise around circle (2). The map $f: X \rightarrow S^{1}$ is induced by the projection of $Y$ on the interval $[0,2 \pi]$.

The critical angles. Clearly the critical angles of $f$ are

$$
\left\{\theta_{0}=0=2 \pi, \theta_{1}, \theta_{2}, \theta_{2}, \theta_{3}, \theta_{4}, \theta_{5}, \theta_{6}\right\} .
$$

The Jordan blocks. The $r$-monodromy of $f$ calculated at $\theta=0$ is given by the regular part of the linear relation $R\left(A_{r}, B_{r}\right)$ with $A_{r}:=\phi_{r}: H_{r}\left(Y_{1}\right) \rightarrow H_{r}(Y)$ induced by $\phi$ and $B_{r}:=i_{r}: H_{r}\left(Y_{1}\right) \rightarrow H_{r}(Y)$ induced by the inclusion $Y_{1} \subset$ $Y$. Since $H_{2}\left(Y_{1}\right)=0$ there is no monodromy for $r=2$ and for $r=0$ one has $R_{\text {reg }}\left(A_{0}, B_{0}\right)=$ Id which leads to

$$
\mathcal{J}_{0}(f)=\{(1,1)\} .
$$

For $r=1$ the reader can see from the picture above that $H_{1}\left(Y_{1}\right)=\kappa^{3}$ generated by the circles $1,2,3$, and $H_{1}(Y)=\kappa^{4}$ generated by the circles $1,2,3$, and an 


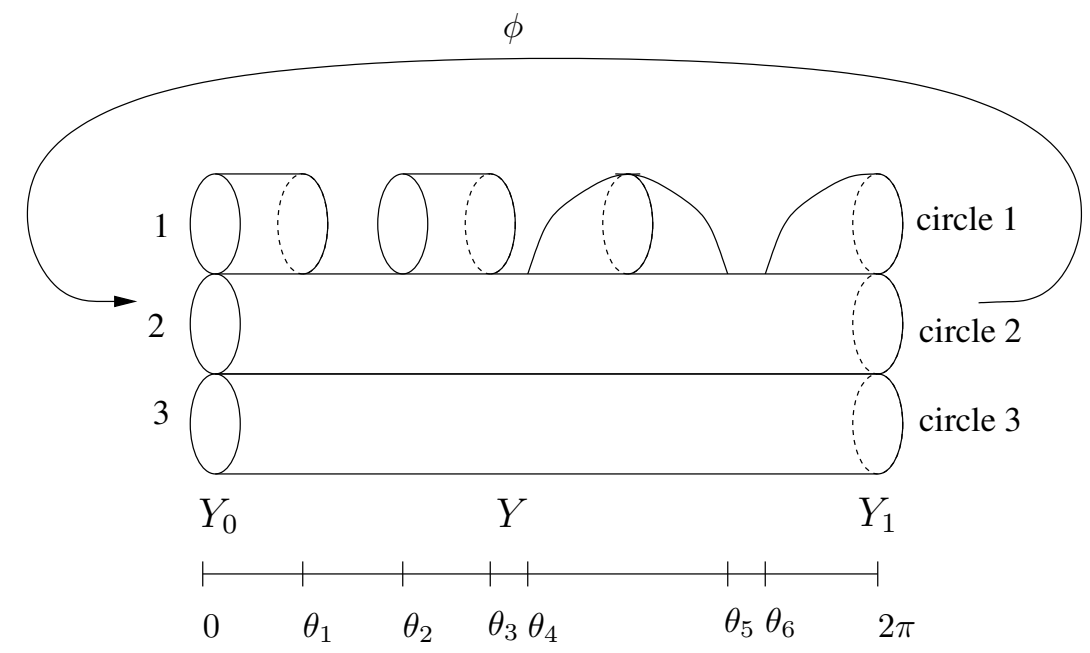

FIgURE 5. Example of $r$-invariants for a circle valued map.

additional generator coming from the small cylinder above $\left[\theta_{2}, \theta_{3}\right]$. In this case

$$
A_{1}=\left(\begin{array}{ccc}
3 & 3 & 0 \\
2 & 3 & -1 \\
1 & 2 & 3 \\
0 & 0 & 0
\end{array}\right) \quad \text { and } \quad B_{1}=\left(\begin{array}{ccc}
0 & 0 & 0 \\
0 & 1 & 0 \\
0 & 0 & 1 \\
0 & 0 & 0
\end{array}\right) \text {. }
$$

Let

$$
A=\left(\begin{array}{ccc}
3 & 3 & 0 \\
2 & 3 & -1 \\
1 & 2 & 3
\end{array}\right) \quad \text { and } \quad B=\left(\begin{array}{ccc}
0 & 0 & 0 \\
0 & 1 & 0 \\
0 & 0 & 1
\end{array}\right)
$$

In view of Observation 8.7 one has $R\left(A_{1}, B_{1}\right)=R(A, B)$, and since $A$ is invertible,

$$
R_{\text {reg }}\left(A_{1}, B_{1}\right)=R_{\text {reg }}(A, B)=R_{\text {reg }}\left(\mathrm{Id}, A^{-1} B\right)=\left(R_{\text {reg }}\left(A^{-1} B, \text { Id }\right)\right)^{-1} \cong\left(\begin{array}{ll}
2 & 1 \\
0 & 2
\end{array}\right),
$$

hence

$$
\mathcal{J}_{1}(f)=\{(2,2)\}
$$

The bar codes. In view of Proposition 4.2(b) by inspections of $f^{-1}\left(\left[\theta, \theta^{\prime}\right]\right)$ one concludes that $\mathcal{B}_{0}(f)=\emptyset, \mathcal{B}_{2}(f)=\emptyset$, and in dimension 1 one has: one closed bar code $\left[\theta_{2}, \theta_{3}\right]$; one open bar code $\left(\theta_{4}, \theta_{5}\right)$; and one open-closed bar code $\left(\theta_{6}, \theta_{1}+2 \pi\right]$.

\section{Appendix B. More examples}

The three examples below support the comments (a), (b), and (c) about the differences between the stability results Theorems 1.2 and 1.5 in the introduction. In all three examples we construct a 1-parameter family of maps, $f_{\epsilon}: X \rightarrow \mathbb{S}^{1}$, and analyze the dependence of the barcode structure on the parameter $\epsilon$.

As in Appendix A the maps in these examples are derived from maps $\bar{f}_{\epsilon}: Y \rightarrow$ $[0,2 \pi]$ by identifying $\bar{f}_{\epsilon}^{-1}(0)$ with $\bar{f}_{\epsilon}^{-1}(2 \pi)$ and 0 with $2 \pi$, respectively. 


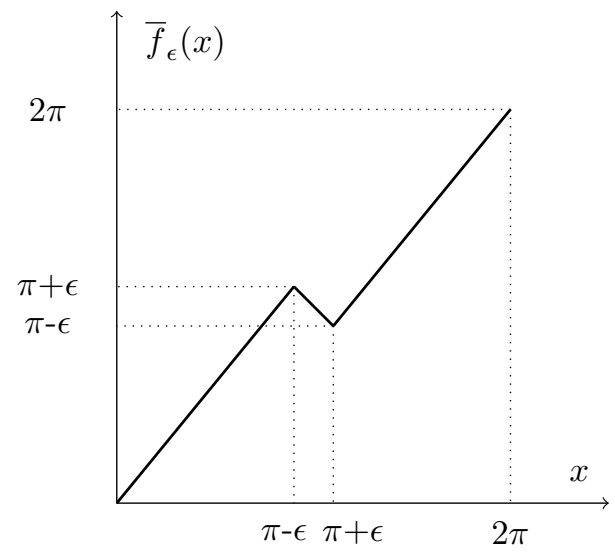

Figure 6. The graph of the map $\bar{f}_{\epsilon}:[0,2 \pi] \rightarrow[0,2 \pi]$ in Example B.1.

Example B.1. Take $Y=[0,2 \pi]$. For $0 \leq \epsilon<\pi$ define a map $\bar{f}_{\epsilon}:[0,2 \pi] \rightarrow[0,2 \pi]$ by

$$
\bar{f}_{\epsilon}(x):= \begin{cases}\frac{\pi+\epsilon}{\pi-\epsilon} x & \text { if } 0 \leq x \leq \pi-\epsilon, \\ -x+2 \pi & \text { if } \pi-\epsilon \leq x \leq \pi+\epsilon, \text { and } \\ \frac{\pi+\epsilon}{\pi-\epsilon} x-\frac{4 \pi \epsilon}{\pi-\epsilon} & \text { if } \pi+\epsilon \leq x \leq 2 \pi .\end{cases}
$$

Figure 6 displays the graph of $\bar{f}_{\epsilon}$. Clearly, $f_{\epsilon}$ can be made is arbitrary closed to $f_{0}$. For $\epsilon=0$ there are no barcodes and only one Jordan cell, $\mathcal{J}_{0}\left(f_{0}\right)=\left\{\left(\kappa^{1}\right.\right.$,id $\left.)\right\}$. For $0<\epsilon<\pi$ we have the same Jordan block, $\mathcal{J}_{0}\left(f_{\epsilon}\right)=\left\{\left(\kappa^{1}\right.\right.$, id $\left.)\right\}$, and in addition two barcodes, $\mathcal{B}_{0}\left(f_{\epsilon}\right)=\{[\pi-\epsilon, \pi+\epsilon),(\pi-\epsilon, \pi+\epsilon]\}$.

Example B.2. Take $Y=Y_{1} \cup Y_{2} \cup Y_{3} \subseteq \mathbb{R}^{2}$, where

$$
\begin{aligned}
& Y_{1}=\{(x, 0) \mid 0 \leq x \leq 2 \pi\}, \\
& Y_{2}=\left\{(x, y) \mid(x-\pi / 2)^{2}+y^{2}=(\pi / 4)^{2}, y \geq 0\right\}, \text { and } \\
& Y_{3}=\{(x, y) \mid y=x-\pi, 5 \pi / 4 \leq x \leq 7 \pi / 4\} .
\end{aligned}
$$

For $0 \leq \epsilon \leq \pi / 4$ define $\bar{f}_{\epsilon}: Y \rightarrow[0,2 \pi]$ as composition $\bar{f}_{\epsilon}=l_{\epsilon} \circ p$, where $p: Y \rightarrow$ $[0,2 \pi]$ denotes the coordinate projection given by $p(x, y)=x$ and $l_{\epsilon}:[0,2 \pi] \rightarrow$ $[0,2 \pi]$ is the piecewise linear map defined by

$$
l_{\epsilon}(t):= \begin{cases}\frac{t}{\pi / 4}(\pi / 4+\epsilon) & \text { if } 0 \leq t \leq \pi / 4, \\ \frac{3 \pi / 4-t}{\pi / 2}(\pi / 4+\epsilon)+\frac{t-\pi / 4}{\pi / 2}(3 \pi / 4-\epsilon) & \text { if } \pi / 4 \leq t \leq 3 \pi / 4, \\ \frac{5 \pi / 4-t}{\pi / 2}(3 \pi / 4-\epsilon)+\frac{t-3 \pi / 4}{\pi / 2}(5 \pi / 4+\epsilon) & \text { if } 3 \pi / 4 \leq t \leq 5 \pi / 4, \\ \frac{7 \pi / 4-t}{\pi / 2}(5 \pi / 4+\epsilon)+\frac{t-5 \pi / 4}{\pi / 2}(7 \pi / 4-\epsilon) & \text { if } 5 \pi / 4 \leq t \leq 7 \pi / 4, \text { and } \\ \frac{2 \pi-t}{\pi / 4}(7 \pi / 4-\epsilon)+\frac{t-7 \pi / 4}{\pi / 4} 2 \pi & \text { if } 7 \pi / 4 \leq t \leq 2 \pi .\end{cases}
$$

The graph of the map $l_{\epsilon}$ is displayed in Figure 7 , the space $Y$ and the map $\bar{f}_{\epsilon}$ are indicated in Figure 8. For $0 \leq \epsilon<\pi / 4$ we have one Jordan cell, $\mathcal{J}_{0}\left(f_{\epsilon}\right)=\left\{\left(\kappa^{1}\right.\right.$, id $\left.)\right\}$, two 0-barcodes, and no 1-barcodes, that is, $\mathcal{B}_{0}\left(f_{\epsilon}\right)=\{(\pi / 4+\epsilon, 3 \pi / 4-\epsilon),(5 \pi / a+$ $\epsilon, 7 \pi / 4-\epsilon]\}$. For $\epsilon=\pi / 4$ we have the same Jordan cell, $\mathcal{J}_{0}\left(f_{\pi / 4}\right)=\left\{\left(\kappa^{1}\right.\right.$, id $\left.)\right\}$, no 0 -barcodes, and one 1-barcode, $\mathcal{B}_{1}\left(f_{\pi / 4}\right)=\{[\pi / 2, \pi / 2]\}$. 


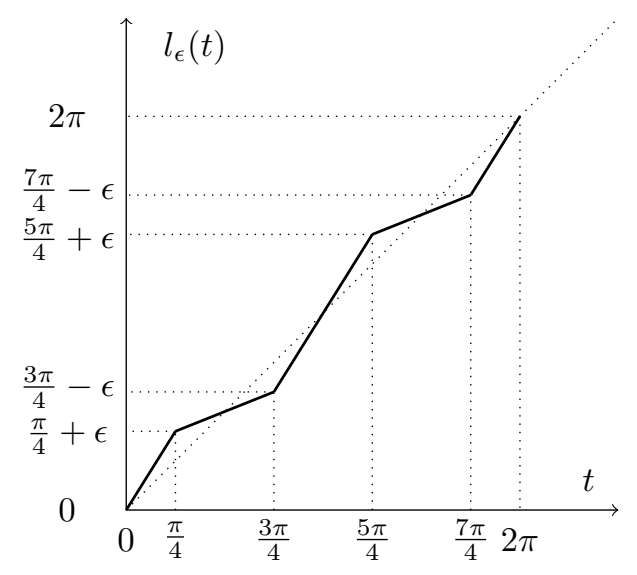

Figure 7. The graph of the map $l_{\epsilon}:[0,2 \pi] \rightarrow[0,2 \pi]$ in Example B.2.

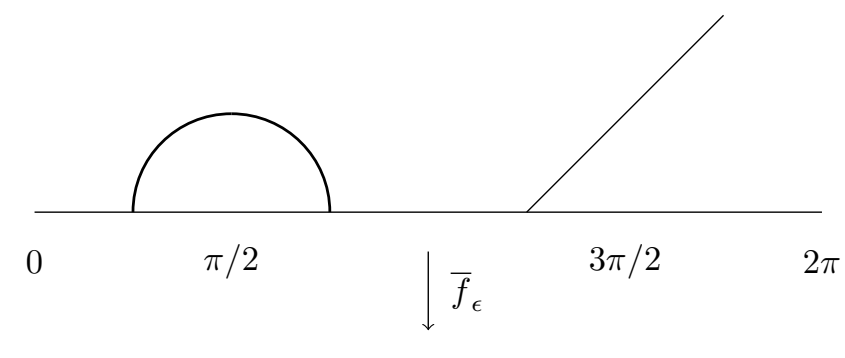

Figure 8. The map $\bar{f}_{\epsilon}: Y \rightarrow[0,2 \pi]$ in Example B.2.

Example B.3. Take $Y=Y_{1} \cup Y_{2} \subseteq[0,2 \pi] \times \mathbb{S}^{1}$ where $Y_{1}=[0,2 \pi] \times\{p\}, Y_{2}=$ $[2 \pi / 3,4 \pi / 3] \times \mathbb{S}^{1}$, and $p \in \mathbb{S}^{1}$ is a base point. For $0 \leq \epsilon<4 \pi / 3$ we define a map $\bar{f}_{\epsilon}: Y \rightarrow[0,2 \pi]$ by

$$
\bar{f}_{\epsilon}(x, y):= \begin{cases}\frac{2 \pi+3 \epsilon}{2 \pi} x & \text { if } 0 \leq x \leq 2 \pi / 3 \\ \frac{2 \pi-6 \epsilon}{2 \pi} x+3 \epsilon & \text { if } 2 \pi / 3 \leq x \leq 4 \pi / 3, \text { and } \\ \frac{2 \pi+3 \epsilon}{2 \pi} x-3 \epsilon & \text { if } 4 \pi / 3 \leq x \leq 2 \pi .\end{cases}
$$

The space $Y$ and the map $\bar{f}_{\epsilon}$ are illustrated in Figure 9. For $0 \leq \epsilon \leq \pi / 3$ we have one Jordan cell, $\mathcal{J}_{0}\left(f_{\epsilon}\right)=\left\{\left(\kappa^{1}\right.\right.$, id $\left.)\right\}$, no 0-barcodes, and one 1-barcode, $\mathcal{B}_{1}\left(f_{\epsilon}\right)=$ $\{[2 \pi / 3+\epsilon, 4 \pi / 3-\epsilon]\}$. For $\pi / 3<\epsilon<4 \pi / 3$ we have the same Jordan cell, $\mathcal{J}_{0}\left(f_{\epsilon}\right)=$ $\left\{\left(\kappa^{1}, \mathrm{id}\right)\right\}$, two 0-barcodes and one 1-barcode, that is, $\mathcal{B}_{0}\left(f_{\epsilon}\right)=\{[4 \pi / 3-\epsilon, 2 \pi / 3+$ $\epsilon),(4 \pi / 3-\epsilon, 2 \pi / 3+\epsilon]\}$, and $\mathcal{B}_{1}\left(f_{\epsilon}\right)=\{[4 \pi / 3-\epsilon, 2 \pi / 3+\epsilon]\}$.

Denote by $f_{\epsilon}^{X_{1}}: X_{1} \rightarrow \mathbb{S}^{1}$ and $f_{\epsilon}^{X_{2}}: X_{2} \rightarrow \mathbb{S}^{1}$ the maps $f_{\epsilon}: X \rightarrow \mathbb{S}^{1}$ described in Examples B.2 and B.3, respectively. Moreover, let $Q$ denote the Hilbert cube, that is, the product of countably many copies of the unit interval. Note that $X_{1} \times Q$ and $X_{2} \times Q$ are homeomorphic compact ANRs. Moreover, for $i=1,2$ we have $\mathcal{B}_{r}\left(f_{\epsilon}^{X_{i}}: X_{i} \rightarrow \mathbb{S}^{1}\right)=\mathcal{B}_{r}\left(f_{\epsilon}^{X_{i}} \circ p_{i}: X_{i} \times Q \rightarrow \mathbb{S}^{1}\right)$ where $p_{i}: X_{i} \times Q \rightarrow X_{i}$ denotes the canonical projection. Hence, one can clearly provide four homotopic tame 

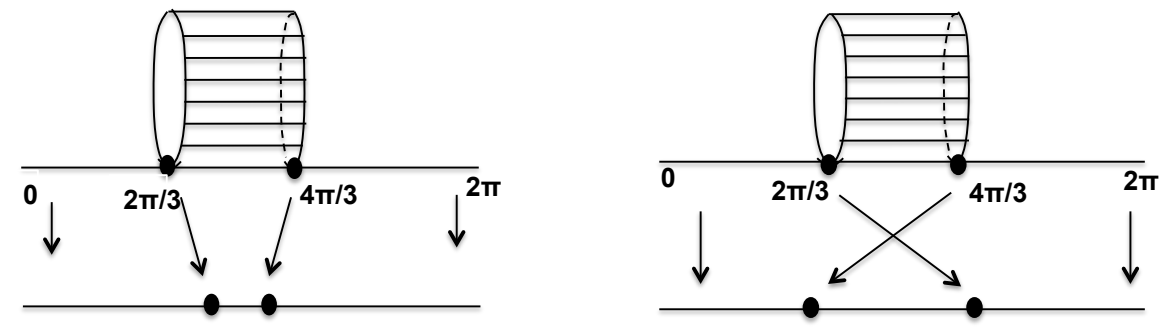

Figure 9. The map $\bar{f}_{\epsilon}: Y \rightarrow[0,2 \pi]$ in Example B.3 for $0 \leq \epsilon<$ $\pi / 3$ (left) and $\pi / 3<\epsilon<4 \pi / 3$ (right).

maps, $h_{1}, h_{2}, h_{3}, h_{4}: X_{1} \times Q \rightarrow \mathbb{S}^{1}$, with $\mathcal{B}_{r}\left(h_{1}\right)=\mathcal{B}_{r}\left(f_{0}^{X_{1}}\right), \mathcal{B}_{r}\left(h_{2}\right)=\mathcal{B}_{r}\left(f_{\pi / 4}^{X_{1}}\right)$, $\mathcal{B}_{r}\left(h_{3}\right)=\mathcal{B}_{r}\left(f_{\pi / 3}^{X_{2}}\right)$, and $\mathcal{B}_{r}\left(h_{4}\right)=\mathcal{B}_{r}\left(f_{2 \pi / 3}^{X_{2}}\right)$.

Appendix C. Structure of Finitely Generated modules over Principal IDEAL DOMAINS

In this appendix we recall basic facts about modules over principal ideal domains, and we provide more specific information about modules over the principal ideal domain of Laurant polynomials, $\kappa\left[t^{-1}, t\right]$.

Recall that an integral domain is a commutative ring with unit $1 \neq 0$ which has no zero divisors. An integral domain $R$ is called principal ideal domain (PID) if every ideal $I \subseteq R$ is generated by a single element $a \in R$, that is, $I=R a$. Familiar examples of principal ideal domains are $\mathbb{Z}$, the ring of integers; $\kappa[t]$, the ring of polynomials of one variable $t$ with coefficients in a field $\kappa$; and $\kappa\left[t^{-1}, t\right]$, the ring of Laurent polynomials of one variable $t$ with coefficients in the field $\kappa$.

Let $M$ be a module over a principal ideal domain $R$. Recall that $M$ is called free if it admits a basis $\left\{x_{i}\right\}_{i \in I}$, i.e. if it is isomorphic to $\bigoplus_{i \in I} R$ for some index set $I$. In this case, the cardinality of the basis is uniquely determined and referred to as the dimension of the free $R$-module $M$, see [25, Chapter III $\$ 7]$.

A proof of the following basic fact can be found in [25, Theorem III.7.3] or [22, Theorem I.5.1].

Theorem C.1. Suppose $M$ is a submodule of a free module $F$ over a principal ideal domain. Then $M$ is free and its dimension is at most the dimension of $F$.

The preceeding result readily implies that submodules of finitely generated modules over a principal ideal domain are finitely generated, see [25, Corollary III $\$ 7.2]$.

Let $M$ be a module over a principal ideal domain $R$. The torsion submodule of $M$ is defined to be the submodule of all torsion elements, $\operatorname{Tor}(M):=\{x \in M \mid$ $\exists \lambda \in R \backslash 0$ such that $\lambda x=0\}$. If $\operatorname{Tor}(M)=0$, then $M$ is called torsion free. If $\operatorname{Tor}(M)=M$, then $M$ is called torsion module.

We have the following fundamental structure theorem, see [25, Theorem III§7.3].

Theorem C.2. If $M$ is a finitely generated module over a principal ideal domain, then $M / \operatorname{Tor}(M)$ is free, and there exists an isomorphism $M \cong \operatorname{Tor}(M) \oplus$ $(M / \operatorname{Tor}(M))$. 
In other words, if $M$ is a finitely generated module over a principal ideal domain $R$, then there exists a decomposition $M \cong T \oplus F$ where $T$ is a finitely generated torsion module and $F$ is a finite dimensional free module. Moreover, the summands $T$ and $F$ are uniquely determined, up to isomorphism. More precisely, $T \cong \operatorname{Tor}(M)$ and $F \cong R^{m}$ where $m \in \mathbb{N}_{0}$ denotes the dimension of $M / \operatorname{Tor}(M)$.

Let us now consider the pricipal ideal domain $R=\kappa\left[t^{-1}, t\right]$ where $\kappa$ is a field. A module over this ring is exactly the same thing as a pair $(M, T)$ where $M$ a $\kappa$-vector space and $T: M \rightarrow M$ is a $\kappa$-linear isomorphism. The vector space $M$ is the underlying vector space of the module and the $\kappa$-linear isomorphism $T$ is defined by multiplication by $t$, its inverse being the multiplication by $t^{-1}$. Note that $M$ is a finite dimensional $\kappa$-vector space if and only if the module is a finitely generated torsion module. Hence, finitely generated torsion modules over $\kappa\left[t^{-1}, t\right]$ can equivalent be regarded as pairs $(M, T)$ where $M$ is a finite dimensional $\kappa$-vector space and $T: M \rightarrow M$ is an isomorphism.

If $M$ is a finitely generated module over $\kappa\left[t^{-1}, t\right]$, then its torsion submodule coincides with the kernel of the homomorphism obtained by tensorizing the natural inclusion $\left.\kappa\left[t^{-1}, t\right] \subseteq \kappa\left[t^{-1}, t\right]\right]$ with $M$, that is,

$$
\left.\operatorname{Tor}(M)=\operatorname{ker}\left(M \rightarrow \kappa\left[t^{-1}, t\right]\right] \otimes_{\kappa\left[t^{-1}, t\right]} M\right) .
$$

Here $\left.\kappa\left[t^{-1}, t\right]\right]$ denotes the field of Laurant series in one variable.

Consider a $G_{2 m}$-representation $\rho$ and a decomposition

$$
\rho \cong \bigoplus_{I \in \mathcal{B}(\rho)} \rho_{I} \oplus \bigoplus_{J \in \mathcal{J}(\rho)} \rho_{J}
$$

as in Section 2 Then the infinite cyclic covering $\tilde{\rho}$ is a finitely generated module over $\kappa\left[t^{-1}, t\right]$ in a natural way. Its free part is isomorphic to the vector space $\kappa[\widetilde{\mathcal{B}}(\rho)]$ equipped with the isomorphism $T$ induced by the translation $\tau(I)=I+2 \pi$. Its torsion part is isomorphic to the pair $(V, T)$ where $V=\bigoplus_{J \in \mathcal{J}(\rho)} V_{J}$ and $T=$ $\bigoplus_{J \in \mathcal{J}(\rho)} T_{J}$. Clearly $V=\kappa[\tilde{\mathcal{J}}(\rho)]$. The underling vector space of this module is $\kappa[\widetilde{\mathcal{B}}(\rho) \sqcup \widetilde{\mathcal{J}}(\rho)]$.

\section{REFERENCES}

[1] M. Atiyah, On the Krull-Schmidt theorem with application to sheaves, Bull. Soc. Math. France 84(1956), 307-317.

[2] I. Bucur and A. Deleanu, Introduction to the theory of categories and functors. With the collaboration of Peter J. Hilton and Nicolae Popescu. Pure and Applied Mathematics, Vol. XIX Interscience Publication John Wiley \& Sons, Ltd., London-New York-Sydney 1968.

[3] D. Burghelea and T. K. Dey, Topological persistence for circle-valued maps, Discrete and Comput. Geom. 50(2013), 69-98.

[4] D. Burghelea and S. Haller, Dynamics, Laplace transform and spectral geometry, J. Topol. 1(2008), 115-151.

[5] D. Burghelea, A refinement of Betti numbers in the presence of continuous real valued function, (I). To appear in Algebraic and Geometric Topology (2017). Preprint available at arXiv:1501.01012

[6] D. Burghelea, A refinement of Betti numbers and homology in the presence of a continuous function II (the case of an angle valued map). Preprint available at arXiv:1603.01861

[7] D. Burghelea, Linear relations, monodromy and Jordan cells of a circle valued map. Preprint available at arXiv:1501.02486

[8] D. Burghelea, New topological invariants for real valued and angle valued maps. An alternative to Morse-Novikov theory. World Scientific Publishing Co. Pte. Ltd., Hackensack, NJ, 2018. (to appear) 
[9] A. Borel and J. C. Moore, Homology theory for locally compact spaces, Mich. Journal of Math. 7(1959), 137-159.

[10] G. Carlsson, V. de Silva and D. Morozov, Zigzag persistent homology and real-valued functions, Proc. of the 25th Annual Symposium on Computational Geometry 2009, 247-256.

[11] D. Cohen-Steiner, H. Edelsbrunner, and J. L. Harer, Stability of persistence diagrams, Discrete Comput. Geom. 37(2007), 103-120.

[12] D. Cohen-Steiner, H. Edelsbrunner, and J. L. Harer, Extending persistence using Poincaré and Lefschetz duality, Found. Comput. Math. 9(2009), 79-103.

[13] R. Deheuvels, Topologie d'une fonctionelle, Ann. of Math. 61(1955), 13-72.

[14] H. Derksen and J. Weyman, Quiver Representations, Notices Amer. Math. Soc. 52(2005), 200-206.

[15] P. Donovan and M. R. Freislich, The representation theory of finite graphs and associated algebras, Carleton Mathematical Lecture Notes 5, Carleton University, Ottawa, 1973.

[16] H. Edelsbrunner, J. L. Harer, Computational topology: An Introduction. AMS, Providence, RI, 2009.

[17] H. Edelsbrunner, D. Letscher, and A. Zomorodian, Topological persistence and simplification, Discrete Comput. Geom. 28(2002), 511-533.

[18] M. Farber, Topology of closed one-forms. Mathematical Surveys and Monographs 108, American Mathematical Society, 2004.

[19] M. Ferri, C. Landi, Representing size functions by complex polynomials, Proc. Math. Met. in Pattern Recognition 9, Moskow, November 16-19, 1999. 18

[20] P. Gabriel, Unzerlegbare Darstellungen I, Manuscripta Math. 6(1972), 71-103.

[21] A. Hatcher, Algebraic topology. Cambridge University Press, Cambridge, 2002.

[22] P. J. Hilton and U. Stammbach, A Course in Homological Algebra. Second edition. Graduate Texts in Mathematics 4. Spinger Verlag, 1996.

[23] S. Hu, Theory of Retracts. Wayne State University Press, Detroit, 1965.

[24] M. Hutchings and Y.-J. Lee, Circle-valued Morse theory, Reidemeister torsion, and SeibergWitten invariants of 3-manifolds, Topology 38(1999), 861-888.

[25] S. Lang, Algebra. Revised third edition. Graduate Texts in Mathematics 211. SpringerVerlag, New York, 2002.

[26] J. Milnor, Morse theory. Princeton Univ. Press, Princeton N.J., 1963.

[27] J. Milnor, Lectures on h-Cobordism Theorem. Notes by L. Siebenmann and J. Sondow. Princeton Univ. Press, Princeton N.J., 1965.

[28] M. Morse, Rank and span in functional topology, Ann. of Math. 41(1940), 419-454.

[29] L. A. Nazarova, Representations of quivers of infinite type (Russian), Izv. Akad. Nauk SSSR Ser. Mat. 37(1973), 752-791.

[30] S. P. Novikov, Quasiperiodic structures in topology. In Topological methods in modern mathematics, Proc. Sympos. in honor of John Milnor's sixtieth birthday, New York, 1991. eds L. R. Goldberg and A. V. Phillips, Publish or Perish, Houston, TX, 1993, 223-233.

[31] A. V. Pajitnov, Circle valued Morse Theory. De Gruyter Studies in Mathematics 32, 2006.

[32] N. Popescu, Abelian categories with applications to rings and modules. London Mathematical Society Monographs, No. 3. Academic Press, London-New York, 1973.

[33] A. Sandovici, H. de Snoo and H. Winkler, The structure of linear relations in Euclidean spaces, Linear Algebra Appl. 397(2005), 141-169.

Dept. of Mathematics, The Ohio State University, 231 West 18th Avenue, Columbus, OH 43210, USA.

E-mail address: burghele@mps.ohio-state.edu

Vienna, Austria.

E-mail address: stefan.haller.42@gmail.com

\footnotetext{
${ }^{18}$ It was brought to our attention that "monic polynomials" to encode persistence diagram were used also in this paper and the additional work quoted in its references; however for a different type of bar codes
} 